\title{
Métodos de pontos interiores aplicados ao pré-despacho de um sistema hidroelétrico usando o princípio de mínimo esforço - comparação com o modelo de fluxo em redes
}

\author{
Lilian Milena Ramos Carvalho \\ Orientador: Prof. Dr. Aurelio Ribeiro Leite de Oliveira
}

Tese apresentada ao Instituto de Ciências Matemáticas e de Computação - ICMC-USP, como parte dos requisitos para obtenção do título de Doutor em Ciências - Área: Ciências da Computação e Matemática Computacional.

USP - São Carlos - SP

Novembro/2005 
Essa tese de doutorado foi desenvolvida com o apoio (bolsa de estudo e reserva técnica) da Fundação de Amparo à Pesquisa do Estado de São Paulo - FAPESP, processo $n^{o} 01 / 13653-5$. 
"Trago dentro do meu coração, Como num cofre que se não pode fechar de cheio, Todos os lugares onde estive, Todos os portos a que cheguei, Todas as paisagens que vi através de janelas ou vigias, Ou de tombadilhos, sonhando, E tudo isso, que é tanto, É pouco para o que eu quero". Fernando Pessoa 
Ao meu marido, Edson Rodrigues Carvalho, que mesmo nas noites de insônia e angústia, manteve-se incondicionalmente ao meu lado, deixando de lado seus sonhos e ajudando-me a buscar os meus. Dedico. 


\section{Agradecimentos}

- Ao Professor Dr. Aurelio Ribeiro Leite de Oliveira, pela orientação precisa, pela confiança e tolerância demonstradas, que foram fundamentais para a conclusão deste trabalho.

- Agradeço aos professores dos cursos de pós-graduação do Instituto de Ciências Matemáticas e de Computação da USP (ICMC) e EESC - Campus de São Carlos, especificamente àqueles com os quais convivi ao longo do curso, pela experiência e conceitos transmitidos, que contribuíram para a conclusão deste trabalho. Em particular, agradeço ao Professor Dr. Marcos Nereu Arenales pela presteza e apoio sempre que solicitado.

- As funcionárias da seção de pós-graduação do ICMC pelo tratamento sempre solícito e pela eficiência no atendimento.

- Aos colegas da pós graduação pelos momentos importantes que compartilhamos.

- A meus pais, Walter e Maria, que estiveram sempre ao meu lado, quer fosse nos momentos bons, quer fosse nos momentos ruins, e sem cujo apoio, teria sido muito difícil alcançar esse objetivo. A eles, quero externar meu mais profundo respeito e admiração.

- À Fundação de Amparo à Pesquisa do Estado de São Paulo - FAPESP, pelo auxílio financeiro dado a esta pesquisa.

- Àqueles que não foram citados, mas de alguma forma, contribuíram para a realização deste trabalho. 


\section{Resumo}

Neste trabalho, os métodos de pontos interiores primal-dual e preditor corretor são estudados e desenvolvidos para o problema de minimização de custos na geração e perdas na transmissão do pré-despacho DC (fluxo de carga em corrente contínua) de um sistema de potência hidroelétrico, com base no modelo de fluxo em redes e no princípio do mínimo esforço. A estrutura matricial, resultante da simplificação do problema proposto pela inclusão do princípio do mínimo esforço, é estudada visando implementações eficientes.

Um método primal-dual perturbado é proposto com base na definição de uma heurística que determinará a escolha do parâmetro de perturbação. Este método mostrou-se eficiente na prática e convergiu em menos iterações quando comparado com uma implementação existente do modelo de fluxo em redes. 


\section{Abstract}

In this work, the primal-dual and preditor corrector interior points methods are studied and developed for the predispatch DC problem that minimizes generation and transmission losses on hydroelectric power systems, on the basis of the network flow model and the minimum effort principle. The matrix structure, resulting of the simplification of the problem considered by inclusion of the minimum effort principle, is studied aiming at

efficient implementations. A disturbed primal-dual method is considered on the basis of a heuristic definition that determine the choice of the disturbance parameter. This method showed to be efficient in practice and converged in fewer iterations when compare with an existing implementation of the network flow model. 


\section{Conteúdo}

1 Introdução 1

1.1 Fluxo de Potência Ótimo . . . . . . . . . . . . . . . . . . 1

1.2 O problema do Pré-Despacho . . . . . . . . . . . . . . . . 2

1.3 Métodos de Pontos Interiores . . . . . . . . . . . . . . . 5

1.4 Objetivos e contribuições deste Trabalho . . . . . . . . . . . . . . . . . . . 10

1.5 Estrutura do Trabalho . . . . . . . . . . . . . . . . . . . . 10

2 O Modelo de Fluxo de Carga DC e o Princípio do Mínimo Esforço 12

2.1 Introdução . . . . . . . . . . . . . . . . . . . . . . . . . 12

2.2 Modelo de Fluxo de Carga Linearizado . . . . . . . . . . . . . . . . . . . . 12

2.3 O Modelo Matemático do Fluxo de Potência Ótimo . . . . . . . . . . . . . 13

2.4 O Princípio do Mínimo Esforço . . . . . . . . . . . . . . . . . . . . . 17

2.5 Conclusão . . . . . . . . . . . . . . . . . . . . . . . . . . . 18

3 Métodos de Pontos Interiores $\quad 20$

3.1 Introdução . . . . . . . . . . . . . . . . . . . . . . . . . . . . 20

3.2 O Problema da Otimização . . . . . . . . . . . . . . . . . . . 21

3.2.1 Diferentes Formas de Otimização . . . . . . . . . . . . . . . . . . 21

3.2 .2 Programação Linear . . . . . . . . . . . . . . . . . . . . . . 22

3.2 .3 Programação Quadrática . . . . . . . . . . . . . . 23

3.2.4 Programação Quadrática Convexa . . . . . . . . . . . . 23

3.3 Multiplicadores de Lagrange . . . . . . . . . . . . . . . . . . . . . . . . . 24

3.4 O Problema da Barreira Logarítmica . . . . . . . . . . . . . . . . . 26

3.5 A Existência e Unicidade de Solução para o Problema da Barreira . . . . . 29 
3.6 Condições de Otimalidade para os Problemas Primal e Dual . . . . . . . . 29

3.7 Métodos Primais-Duais . . . . . . . . . . . . . . . . . 31

3.7.1 O Caminho Central . . . . . . . . . . . . . . . . . 33

3.7 .2 Métodos de Trajetória Central . . . . . . . . . . . . . . . 34

3.7.3 Método de Passo Curto . . . . . . . . . . . . . . . . . 36

3.7.4 Um Teorema de Complexidade Geral para Métodos de Trajetória

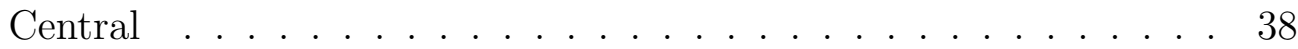

3.8 Método de Passo Longo . . . . . . . . . . . . . . . . . . . . . 39

3.8.1 Métodos de Pontos Interiores Infactíveis . . . . . . . . . . . . . . . 42

3.8.2 Método Preditor-Corretor . . . . . . . . . . . . . . 44

3.9 Conclusão . . . . . . . . . . . . . . . . . . . . . 46

4 Métodos de Pontos Interiores para Problema de Fluxo de Potência $\begin{array}{ll}\text { Ótimo DC } & 47\end{array}$

4.1 Introdução . . . . . . . . . . . . . . . . . . . . . . 47

4.2 O Modelo Dinâmico . . . . . . . . . . . . . . . . . . . . . 48

4.3 Desenvolvimento do Método . . . . . . . . . . . . . . . . . . . 48

4.4 Problema de Pré-Despacho Dual . . . . . . . . . . . . . . . . . . . . . 50

4.5 Condições de Otimalidade . . . . . . . . . . . . . . . . . 53

4.6 Método de Newton . . . . . . . . . . . . . . . . . . . . . . 54

4.7 Redução do Sistema Linear . . . . . . . . . . . . . . . . . . . 56

4.8 Estudo da Estrutura Matricial . . . . . . . . . . . . . . . 58

4.9 Métodos Primal-Dual e Preditor-Corretor . . . . . . . . . . . . . . . . . 63

4.10 Conclusão . . . . . . . . . . . . . . . . . . . . . . . . 64

5 Métodos de Pontos Interiores para Problema de Fluxo de Potência Ótimo DC usando o Princípio do Mínimo Esforço $\quad 65$

5.1 Introdução . . . . . . . . . . . . . . . . . . . . . . 65

5.2 O Modelo de Fluxo em Redes . . . . . . . . . . . . . . . . . 66

5.3 Princípio do Mínimo Esforço . . . . . . . . . . . . . . . . . . . . 66

5.4 Problema de Pré-Despacho Dual . . . . . . . . . . . . . . . . . 69

5.5 Condições de Otimalidade . . . . . . . . . . . . . . . . . . . . 71 
5.7 Redução do Sistema Linear . . . . . . . . . . . . . . . . . 74

5.8 Métodos Primal-Dual e Preditor-Corretor . . . . . . . . . . . . . . . 80

5.9 Modificação no Método PDPME . . . . . . . . . . . . . . . . . 81

5.10 Conclusão . . . . . . . . . . . . . . . . . . . 84

6 Experimentos Numéricos $\quad 85$

6.1 Introdução . . . . . . . . . . . . . . . . . . . . . . . . . 85

6.2 Dados de Entrada . . . . . . . . . . . . . . . . . . 87

6.3 Resultados Obtidos . . . . . . . . . . . . . . . . . . . . . . 89

6.3.1 Eliminação de Variáveis 1 . . . . . . . . . . . . . . . . 89

6.3.2 Eliminação de Variáveis 2 . . . . . . . . . . . . . . . . . . . . . . 90

6.3.3 Análise dos Resultados . . . . . . . . . . . . . . . . . 91

6.4 Conclusão . . . . . . . . . . . . . . . . . . . . . . . 102

7 Conclusões e Perspectivas Futuras 103 


\section{Lista de Figuras}

2.1 Representação de uma rede elétrica . . . . . . . . . . . . . . . . . . . 14

2.2 Representação em grafo da rede elétrica da figura 2.1 . . . . . . . . . . . 15

2.3 Representação da lei das malhas na rede elétrica . . . . . . . . . . . . . . 16

3.1 Iterações do Algoritmo MPC . . . . . . . . . . . . . . . . . . 37

3.2 Iterações do Algoritmo MPL . . . . . . . . . . . . . . . . . . . . . . . . 41

3.3 Iterações do Algoritmo MPIPC . . . . . . . . . . . . . . . . . . 45

6.1 Tempo versus Fator peso . . . . . . . . . . . . . . 86

1 Interpretação geométrica do método de Newton para funções de uma variável108 


\section{Lista de Tabelas}

6.1 Fatores de Carga . . . . . . . . . . . . . . . 86

6.2 Método Primal-Dual - Tempo Computacional - 24h . . . . . . . . . . . . 93

6.3 Método Preditor-Corretor- Tempo Computacional - 24h . . . . . . . . . . . 93

6.4 Método Primal-Dual - Flops - 24h . . . . . . . . . . . . . . . . . . 94

6.5 Método Preditor-Corretor - Flops - 24h . . . . . . . . . . . . . . . 94

6.6 Método Primal-Dual - Iterações - 24h . . . . . . . . . . . . . . . . . 95

6.7 Método Preditor-Corretor - Iterações - 24h . . . . . . . . . . . . . . . . 95

6.8 Método Primal-Dual - Tempo Computacional - 48h . . . . . . . . . 96

6.9 Método Preditor-Corretor - Tempo Computacional - 48h . . . . . . . . . . 96

6.10 Método Primal-Dual - Flops - 48h . . . . . . . . . . . . . . . . . 97

6.11 Método Preditor-Corretor - Flops - 48h . . . . . . . . . . . . . . . . 97

6.12 Método Primal-Dual - Iterações - 48h . . . . . . . . . . . . . . . . 98

6.13 Método Preditor-Corretor - Iterações - 48h . . . . . . . . . . . . . . . . 98

6.14 Método Primal-Dual - Tempo Computacional - 72h . . . . . . . . . . . 99

6.15 Método Preditor-Corretor - Tempo Computacional - 72h . . . . . . . . . 99

6.16 Método Primal-Dual - Flops - 72h . . . . . . . . . . . . . . 100

6.17 Método Preditor-Corretor - Flops - 72h . . . . . . . . . . . . . . . 100

6.18 Método Primal-Dual - Iterações - 72h . . . . . . . . . . . . . . . 101

6.19 Método Preditor-Corretor - Iterações - 72h . . . . . . . . . . . . . . . . 101 


\section{Capítulo 1}

\section{Introdução}

\subsection{Fluxo de Potência Ótimo}

A aplicação de técnicas de otimização a problemas de operação e planejamento de sistemas de potência tem sido uma área de pesquisa muito ativa nos últimos anos. Fluxo de potência ótimo é um termo genérico que corresponde a uma ampla classe de problemas que busca otimizar uma função objetivo específica satisfazendo restrições provenientes de particularidades físicas e operacionais da rede elétrica (MOMOH; EL-HAWARY; ADAPA, 1999).

O problema de fluxo de potência ótimo tem aplicação em diversos campos de análise e operação de sistemas de potência, tais como despacho econômico, análise de confiabilidade de geração e transmissão, análise de segurança e planejamento da expansão de geração a curto prazo. Na maior parte destas aplicações, adota-se uma representação linearizada (DC) do fluxo de potência com a finalidade de obter-se uma maior simplicidade no tratamento das informações sem, entretanto, comprometer o grau de precisão dos resultados (OLIVEIRA; SOARES, 2000).

Para a solução de problemas relacionados com fluxo de potência ótimo, existem uma série de métodos e técnicas que podem ser utilizadas, tais como:

- Programação não linear.

- Programação Quadrática.

- Solução baseada em condições de otimalidade e método de Newton. 
- Programação Linear.

- Programação Inteira Mista.

- Métodos de Pontos Interiores.

A ênfase neste trabalho derá dada à classe de Métodos de Pontos Interiores que, desde 1984, quando Karmarkar propôs seu algoritmo para programação linear, tem apresentado métodos e implementações sofisticadas no campo da programação linear e, para problemas mais gerais, como programação quadrática convexa, programação semi-definida e problemas não convexos e não lineares, mostrado um excelente nível de desenvolvimento (POTRA; WRIGHT, 2000).

\subsection{O problema do Pré-Despacho}

O pré-despacho de um sistema de potência hidrelétrico é um problema operacional de curto prazo, em que curto prazo significa a operação horária durante um dia até uma semana. Este problema pode ser modelado pela minimização de uma função quadrática com variáveis separáveis, correspondendo ao custo no sistema hidráulico de geração e perdas na transmissão do sistema de potência, sujeita a restrições lineares representando o fluxo de potência ativa (CHIAVEGATO; OLIVEIRA; SOARES, 2001).

Cada intervalo de operação determina um problema de fluxo de potência ótimo linearizado que pode ser representado por um modelo de fluxo em redes.

O sistema de geração é composto por um conjunto de unidades geradoras, enquanto que o sistema de transmissão é representado por um modelo de fluxo de carga de corrente contínua (DC) com limites sobre o fluxo de potência ativa nas linhas. Se o sistema de potência sob consideração estiver conectado a outros sistemas, a troca de potência por meio desta ligação deve ser levada em consideração impondo ao modelo uma restrição de acoplamento (SOARES; SALMAZO, 1997).

Diferentes abordagens para a solução deste problema do pré-despacho têm sido propostas na literatura (OHISHI; SOARES; CARVALHO, 1991; SOARES; SALMAZO, 1997). O modelo apresentado neste trabalho considera sistemas que são predominantemente formados por hidrelétricas. Em tais sistemas, as unidades térmicas podem operar com um 
nível constante durante um dia, pois, as flutuações provocadas pela demanda diferencial são compartilhadas com as unidades hidrelétricas. Neste caso, os custos para iniciar e interromper as unidades de geração, por exemplo, não são como aqueles envolvidos em sistemas predominantemente térmicos (OLIVEIRA; NEPOMUCENO; SOARES, 2005). Além disso, a função objetivo usual relacionada com a minimização de custos nos sistemas térmicos é substituída pela de minimização de perdas na geração hidrelétrica associada com variações nos canais de fuga, perdas nas comportas e eficiência nos geradores de turbinas (SOARES; SALMAZO, 1997).

Assim, um modelo de pré-despacho hidroelétrico para um sistema de potência com $m$ barras, $n$ linhas e $g$ geradores pode ser formulado como (SOARES; SALMAZO, 1997; OLIVEIRA; NEPOMUCENO; SOARES, 2003, 2005):

$$
\begin{array}{cr}
\text { minimizar } \frac{\alpha}{2} \sum_{k=1}^{t}\left[\left(f^{k}\right)^{\prime} R f^{k}+r^{\prime} f^{k}\right]+\frac{\beta}{2} \sum_{k=1}^{t}\left[\left(p^{k}\right)^{\prime} Q p^{k}+c^{\prime} p^{k}\right] & \\
\text { sujeito a } & k=1, \cdots, t \\
A f^{k}-E p^{k}=-l^{k}, & k=1, \cdots, t \\
T f^{k}=0, & k=1, \cdots, t \\
f^{\text {min }} \leq f^{k} \leq f^{\max }, & k=1, \cdots, t \\
p^{\text {min }} \leq p^{k} \leq p^{\text {max }}, & \\
\sum_{k=1}^{t} p^{k} & =q .
\end{array}
$$

Neste modelo, $t$ corresponde a um período de planejamento em horas e $E$ é uma matriz de ordem $m \times g$ com cada coluna tendo exatamente um elemento igual a 1 e os demais elementos nulos. Cada linha não nula corresponde a uma barra de geração. Como, na prática, as barras de geração e as de carga não estão ordenadas, a matriz $E$ não necessariamente possui a forma $\left[\begin{array}{ll}I & 0\end{array}\right]^{\prime}$ ou $\left[\begin{array}{ll}0 & I\end{array}\right]^{\prime}$ em que $I$ é uma matriz identidade. Além disso, em cada período $k=1,2, \cdots, t$,

- $f^{k}$ representa o fluxo de potência ativa;

- $p^{k}$ representa a geração de potência ativa;

- $R$ representa a matriz diagonal das resistências das linhas;

- A representa a matriz de incidência da rede de transmissão; 
- $T$ representa a matriz de reatância da rede de transmissão;

- $l^{k}$ representa as demandas de potência ativa;

- $f^{\max }$ e $f^{\min }$ são os limites de fluxo de potência ativa;

- $p^{\max }$ e $p^{\min }$ são os limites de geração de energia hidráulica;

- $Q$ representa a matriz do custo de geração com componentes quadráticos;

- $c$ representa o vetor com componentes lineares do custo de geração;

- $r$ representa o vetor com componentes lineares de perdas na transmissão;

- $\alpha$ e $\beta$ são ponderações dos objetivos a minimizar;

- q representa a meta de geração de energia estabelecida pelo planejamento de longo prazo.

O conjunto de restrições para este problema é linear, em que as equações (1.2) e (1.3) correspondem às leis de Kirchhof e representam a rede de geração / transmissão, enquanto que as equações (1.4) e (1.5) representam as capacidades de geração e transmissão do sistema. A equação (1.6) corresponde a uma restrição de acoplamento que é inerente ao modelo dinâmico. A expressão modelo dinâmico utilizada neste trabalho refere-se à extensão do modelo de fluxo em redes para o problema do pré-despacho, por meio do acréscimo de metas de geração para cada hidrelétrica, metas estas, estabelecidas pelo planejamento de longo prazo, e considerando um problema de fluxo de potência para cada intervalo de operação. As duas componentes da função objetivo (1.1) são quadráticas com variáveis separáveis, sendo que a primeira representa o valor econômico das perdas de transmissão e a segunda representa o custo de geração das usinas, tanto térmicas quanto hidrelétricas (OLIVEIRA; SOARES, 2000; SOARES; SALMAZO, 1997).

Definindo $V^{p \times q}$ como o espaço de todas as matrizes de ordem $p \times q$, as dimensões de cada variável envolvida no modelo, para $k=1,2, \cdots, t$, são dadas por:

$f^{k} \in V^{n \times 1}$

$T \in V^{(n-m+1) \times n}$

$A \in V^{m \times n}$ 


$$
\begin{aligned}
& p^{k} \in V^{g \times 1} \\
& l^{k} \in V^{m \times 1} \\
& p^{\text {min }}, p^{\text {max }} \in V^{g \times 1} \\
& f^{\text {min }}, f^{\text {max }} \in V^{n \times 1} \\
& q \in V^{g \times 1} \\
& R \in V^{n \times n} \\
& Q \in V^{g \times g} \\
& c \in V^{g \times 1} \\
& r \in V^{n \times 1} .
\end{aligned}
$$

\subsection{Métodos de Pontos Interiores}

Os métodos de pontos interiores possuem uma abordagem diferente daquela do método Simplex para programação linear. De fato, a diferença fundamental entre os métodos de pontos interiores e o método Simplex repousa na natureza das soluções tentativas (soluções obtidas em cada iteração). No método Simplex, estas soluções pertencem ao contorno da região factível, enquanto que nos métodos de pontos interiores estas soluções são obtidas percorrendo o interior da região factível até atingir o ótimo. Além disso, no método Simplex o número de iterações tende a crescer com o tamanho do problema, enquanto que nos métodos de pontos interiores não existe esta tendência.

O primeiro método de pontos interiores, com barreira logarítmica, é atribuído a Frisch(1955) e foi exaustivamente estudado por Fiacco e McCormick (FIACCO; MCCORMICK, 1968), com a finalidade de resolver problemas com restrições de desigualdades não lineares. Em 1979, Khachiyan (KHACHIYAN, 1979) apresentou o método dos elipsóides para resolver um problema de programação linear em tempo polinomial; entretanto, este método provou, na prática, ser inferior ao Método Simplex. Mas, a maior descoberta no campo de pesquisa com pontos interiores, ocorreu em 1984, quando Karmarkar (KARMARKAR, 1984) apresentou um novo método de pontos interiores para programação linear, afirmando que podia obter soluções cinqüenta vezes mais rápido do que o Método Simplex para grandes problemas. Seu algoritmo é baseado em transformações projetivas não lineares (QUINTANA; TORRES; PALOMO, 2000). 
Em 1991, Clements et al. (CLEMENTS; DAVIS; FREY, 1991) apresentaram um dos primeiros estudos de pontos interiores aplicados a sistemas de potência. Eles desenvolveram uma técnica de pontos interiores de programação não linear para resolver problemas de estimação de estado de um sistema de potência que facilita a detecção e identificação de dados ruins. A técnica utilizou uma função barreira logarítmica para acomodar as restrições de desigualdade e o método de Newton para resolver as equações resultantes da aplicação das condições de Karusch-Kuhn-Tucker (KKT). O método tem a vantagem de resolver o problema com poucas iterações quando comparado à outras técnicas de programação linear, em que o número de iterações é dependente do sistema. A escolha dos pontos iniciais foi uma limitação do método. Entretanto, o método utilizou a vantagem da decomposição de Cholesky para reduzir o tempo de processamento.

Ainda em 1991, Pannambalam et al. (PANNAMBAlam; QUINTANA; VANNELLI, 1992) apresentaram um método dual-afim que corresponde a uma variante do método de pontos interiores de Karmarkar. Esse método foi implementado para resolver o problema de planejamento de uso da água, formulado como um problema de programação linear com restrições de igualdade e desigualdade. A superioridade do método dual-afim sobre o Método Simplex para o problema em questão ficou estabelecida.

Em 1992, Vargas et al. (VARGAS; QUINTANA; VANNELLI, 1993) apresentaram um método de pontos interiores para resolver o problema de despacho econômico de sistemas de potência. Eles empregaram uma abordagem de programação linear sucessiva para problemas de despacho econômico com restrições de segurança. Neste método, uma nova abordagem de métodos de pontos interiores dual-afim foi utilizada para resolver problemas de programação linear. Com isto, foi resolvido o problema clássico de fluxo de potência ótimo com restrições de fluxo de potência, fluxos, geração ativa e reativa, além da magnitude da tensão. O método de pontos interiores utilizado obteve a solução ótima em poucas iterações comparado ao MINOS 5.0.

Ainda, em 1992, Lu e Unum (LU; UNUM, 1993) apresentaram um método de pontos interiores para resolver problemas de programação linear com controle de segurança restrito a vários tamanhos de redes. O método empregou a técnica de programação linear para obter uma solução inicial factível, antes de aplicar o método de pontos interiores, sendo que o problema original foi resolvido utilizando um método de pontos interiores 
primal. Testes mostraram que o método usa menos tempo de processamento quando comparado com o MINOS 5.0, mas que a convergência pode ser lenta nas últimas iterações do processo. Os estudos feitos mostraram, ainda, que este método de pontos interiores é adaptável a modelos práticos de sistemas de potência.

Neste mesmo ano (1992), Mehrotra (MEHROTRA, November, 1992) publicou um artigo que descreve uma implementação de um método de ponto interior de segunda ordem que se mostrou bastante efetivo e que passou a ser a base da maioria dos códigos relacionados a pontos interiores. O método em questão utiliza um polinômio de Taylor de segunda ordem para aproximar a trajetória primal-dual.

Conforme relata Mehrotra neste artigo, os métodos de pontos interiores primaisduais têm suas raízes no artigo de Megiddo (MEGIDDO, New York, 1986), no qual ele descreve um método primal-dual. Mais tarde, em 1989, Kojima, Mizuno e Yoshise (KOJIMA; MIZUNO; YOSHISE, 1989) e Monteiro e Adler (MONTEIRO; ADLER, 1989) desenvolveram e analisaram este trabalho de Megiddo, mostrando que a trajetória central pode ser percorrida em direção à solução ótima em $O(\sqrt{n} L)$ iterações tomando passos curtos.

Em 1993, Güler e Ye (GULER; YE, 1993) mostraram que a maioria dos algoritmos de pontos interiores para programação linear geram uma seqüência de soluções nas quais todo ponto limite satisfaz a condição de complementaridade estrita. Estes algoritmos incluem todos os algoritmos seguidores de caminho e alguns algoritmos de redução do potencial.

Ainda em 1993, Kojima, Megiddo e Mizuno (KoJIMA; MEGIDdo; MiZuno, 1993) propuseram uma regra para o tamanho do passo segundo o qual os algoritmos podem tomar passos longos e distintos nos espaços primal e dual, mantendo a convergência global.

Nestes algoritmos, a seqüência gerada $\left(x^{k}, y^{k}, z^{k}\right)$ não é restrita ao interior da região factível e nem é exigido que cada termo dela satisfaça as restrições de igualdade $A x=b$ e $A^{\prime} y+z=c$, mas apenas a condição $x>0$ e $z>0$.

No final deste mesmo ano, Mizuno, Todd e Ye (MIZUnO; TODD; YE, November, 1993) descreveram e analisaram vários algoritmos de pontos interiores primais-duais para programação linear. Todos os métodos discutidos têm complexidade polinomial enquanto que alguns permitem passos longos em circunstâncias favoráveis.

Em 1994, Potra (POTRA, 1994) propôs um algoritmo de ponto interior preditor- 
corretor para problemas lineares que iniciam a partir de pontos infactíveis. O método converge quadraticamente, e se a razão entre factibilidade e otimalidade para o ponto inicial é suficientemente pequena, então, o algoritmo tem complexidade da ordem de $\sqrt{n L}$ iterações.

Em 1996, Kojima (KOJIMA, 1996) apresentou alguns lemas básicos aplicados a métodos de pontos interiores infactíveis para problemas lineares que permitem grande flexibilidade e aperfeiçoamento em restrições impostas sobre pontos iniciais e tamanho do passo nos espaços primal e dual. Ele analisa, ainda, a extensão destes lemas para problemas de complementaridade monótona linear e não linear.

Em 2000, Potra e Wright (POTRA; WRIGHT, 2000) apresentaram uma revisão de alguns desenvolvimentos importantes relacionados a algoritmos e softwares para programação linear, bem como suas extensões para classes mais gerais de problemas, tais como programação quadrática convexa, programação semi-definida e problemas não lineares e não convexos.

O trabalho inclui, ainda, comentários sobre teoria da complexidade e métodos práticos para programação linear, programação semi-definida, complementaridade linear monótona e programação convexa sobre conjuntos que podem ser caracterizados por funções barreira auto-concordante.

Em 2001, Peng e Terlaky (PENG; TERLAKY, 2001) restringindo-se à otimização linear, exploraram algumas interessantes propriedades de uma função de medida de proximidade que é chave na definição da vizinhança do caminho central e propuseram um método de ponto interior dinâmico e estabeleceram sua complexidade como sendo da ordem de $n^{\frac{2}{3}} \log \frac{n}{\epsilon}$ iterações.

Ainda, em 2001, Stojkovic e Stanimirovic (STOJOKOVIC; STANIMIROVIC, 2001) propuseram dois algoritmos para a escolha de um ponto inicial eficiente em um algoritmo primal-dual. Implementaram os dois algoritmos no pacote MATHEMATICA e apresentaram vários exemplos numéricos.

Também, em 2001, Castronuovo, Campagnolo e Salgado (CASTRONUOVO; CAMPAGNOLO; SALGADO, November, 2001) mostraram que combinações convenientes da direção afim-escala e centralização pode aumentar o potencial de métodos de otimização em termos de velocidade e confiabilidade. Sistemas testes e redes com tamanhos reais foram 
utilizados para verificar o desempenho do método apresentado.

Em 2005, Silva, Soares e Vicente (SILVA; SOARES; VICENTE, March, 2005), analisaram a taxa de convergência local do método de ponto interior primal-dual factível quando cada ponto na seqüência de iterações é mantido estritamente factível com relação às restrições de desigualdade. Mostraram, ainda, que sob condições clássicas a taxa de convergência é q-quadrática (Apêndice C) quando as funções associadas às restrições de desigualdade definem uma região factível localmente côncava.

Ainda em 2005, Salahi, Peng e Terlaky (SAlAhI; PENG; TERLAKY, April, 2005) analisaram a complexidade polinomial de métodos preditor-corretor do tipo Mehrotra. Consideraram uma variante do protótipo original do método, que tem sido utilizado em várias implementações de métodos de pontos interiores, cujos resultados de complexidade são conhecidos. Utilizando um exemplo, mostraram que nesta variante do tipo Mehrotra, a escolha adaptativa do parâmetro $\mu$ pode forçar o método a tomar vários passos pequenos a fim de manter cada ponto na seqüência em uma certa vizinhança do caminho central, o que é essencial para demonstrar a complexidade polinomial do algoritmo.

Neste mesmo ano, Roos (ROOS, June, 2005) apresentou um algoritmo de ponto interior infactível de passo completo e mostrou que, no máximo, uma ordem de n iterações é suficiente para reduzir o gap de dualidade e os resíduos por um fator de $\frac{1}{\epsilon}$. Supõe, ainda, que investigações maiores poderão aperfeiçoar o limite acima para a ordem de $\sqrt{n}$.

Também em 2005, Oliveira e Sorensen (OLIVEIRA; SORENSEN, 2005) apresentaram uma nova classe de precondicionadores para a solução iterativa de sistemas lineares que surgem da aplicação de métodos de pontos interiores. Mostraram que todo précondicionador para sistema reduzido produz um pré-condicionador equivalente para o sistema aumentado, mas que a inversa não é verdadeira.

Em acréscimo às referências citadas, podemos mencionar uma coleção de artigos relacionados a importantes tópicos em métodos de pontos interiores, e que podem ser encontrados no livro editado por Terlaky (TERLAKY, 1996). Freund e Mizuno (FREUnD; MIZUNO, 1996) fornecem uma sinopse do desenvolvimento de métodos de pontos interiores nos últimos 30 anos, e analisam as pesquisas atuais e seus rumos em métodos de pontos interiores. Apresentam, ainda, uma série de livros textos que contém tratamentos rigorosos sobre métodos de pontos interiores. 


\subsection{Objetivos e contribuições deste Trabalho}

O objetivo deste trabalho consiste em aplicar os métodos de pontos interiores primal-dual e preditor corretor ao problema de minimização de perdas na geração e transmissão do pré-despacho DC de um sistema de potência hidroelétrico baseado no princípio do mínimo esforço e comparar com os resultados obtidos para o modelo de fluxo em redes.

Para satisfazer este objetivo geral, alguns objetivos específicos tiveram que necessariamente ser atingidos no decorrer deste trabalho. São eles:

1. Apresentar o modelo simplificado do problema do pré-despacho devido a inclusão do princípio do mínimo esforço.

2. Estudar a estrutura matricial resultante desta simplificação.

3. Implementar de forma eficiente, tendo em vista o ítem 2 , o modelo simplificado.

4. Comparar os resultados obtidos no ítem 3 com uma implementação do modelo de fluxos em rede já existente.

Neste trabalho, propomos uma versão perturbada de um método primal-dual, cuja novidade consiste na apresentação de uma nova heurística com relação à escolha do parâmetro de perturbação. Além disso, apresentamos duas formas de eliminação de variáveis que, embora levem a um sistema final cuja dimensão é equivalente ao número de geradores do sistema elétrico sob consideração, produzem implementações que não possuem a mesma eficiência do ponto de vista do esforço computacional. De fato, mostramos, pelos resultados obtidos em termos de tempo computacional, número de operações de ponto flutuante e número de iterações, que existe vantagens em, não só reduzir o sistema original, como também explorar diferentes formas de eliminação de variáveis para esta redução.

\subsection{Estrutura do Trabalho}

O presente trabalho encontra-se dividido nos seguintes capítulos: No capítulo 2, são definidos o modelo de fluxo de carga DC e o princípio do mínimo esforço.

No capítulo 3 são fornecidos alguns conceitos sobre otimização e métodos de pontos interiores. 
No capítulo 4, os métodos de pontos interiores primal-dual e preditor corretor são aplicados ao problema de fluxo de potência ótimo DC na formulação de fluxo em redes. É feito um estudo da estrutura matricial resultante visando uma comparação com o modelo simplificado.

No capítulo 5 são feitas simplificações no modelo visto no capítulo 4, por meio da inclusão do princípio do mínimo esforço. Métodos primal-dual e preditor corretor são desenvolvidos e a estrutura da matriz resultante é estudada e comparada com a do modelo de fluxo em redes.

No capítulo 6 são apresentados resultados numéricos relativos à implementação do problema do pré-despacho de um sistema hidroelétrico usando o princípio do mínimo esforço.

O capítulo 7 apresenta a conclusão do trabalho e perspectivas futuras. 


\section{Capítulo 2}

\section{O Modelo de Fluxo de Carga DC e o Princípio do Mínimo Esforço}

\subsection{Introdução}

Conforme delineado em (ALMEIDA, 87), as redes elétricas representadas pelo modelo de fluxo de carga DC e os circuitos resistivos de corrente contínua apresentam a propriedade de que as perdas elétricas entre as linhas ou ramos do circuitos é a mínima possível. Assim, existe uma equivalência entre o problema de fluxo de potência linearizado e o problema de mínimo esforço elétrico em um sistema de transmissão.

O objetivo deste capítulo é apresentar o modelo geral de fluxo de carga linearizado, o modelo matemático do fluxo de potência ótimo aplicado à geração e transmissão de sistemas de potência e o princípio do mínimo esforço.

\subsection{Modelo de Fluxo de Carga Linearizado}

As linhas e os transformadores de uma rede de energia elétrica podem ser representados pelo modelo $\pi$ equivalente. Neste modelo, o fluxo em uma linha $(i, j)$, após algumas hipóteses simplificadoras (MONTICELLI; GARCIA, 1999), pode ser expresso por:

$$
f_{i j}=\frac{\theta_{i}-\theta_{j}}{x_{i j}}
$$


em que $\theta_{i}$ é o ângulo de tensão na barra $i, x_{i j}$ é a reatância na linha $(i, j)$ e $f_{i j}$ o fluxo de potência ativa na linha $(i, j)$.

A equação (2.1) representa uma relação linear entre os fluxos de potência ativa nas linhas e os ângulos de tensões nas barras. Em termos matriciais, para um sistema com $m$ barras e $n$ linhas, a expressão (2.1) pode ser reescrita na forma:

$$
f=X^{-1} A^{\prime} \theta
$$

em que:

- $f$ é o vetor fluxo de potência ativa, de dimensão $n \times 1$.

- X é a matriz diagonal das reatâncias, de dimensão $n \times n$.

- A é a matriz de incidência nó-arco da rede, de dimensão $m \times n$.

- $\theta$ é o vetor dos ângulos de tensões, de dimensão $m \times 1$.

A equação (2.2) é equivalente à segunda lei de Kirchhoff. Por outro lado, a primeira lei de Kirchhof refere-se à conservação de fluxos na rede, ou seja,

$$
\text { Af }=P
$$

em que $P$ é o vetor das injeções líquidas de potência ativa na rede $(P$ tem dimensão $m \times 1)$.

O conjunto constituído pelas equações (2.2) e (2.3) forma o modelo de fluxo de carga linearizado, isto é,

$$
\begin{aligned}
f & =X^{-1} A^{\prime} \theta \\
P & =A f .
\end{aligned}
$$

\subsection{O Modelo Matemático do Fluxo de Potência Ótimo}

Este modelo leva em consideração o cálculo da potência ativa com restrições de geração e transmissão. Ele corresponde a um modelo de fluxo de potência linearizado DC, representado por um modelo de fluxo em redes com restrições adicionais (AZEVEDO, 2002). 
Consideremos a seguinte rede elétrica com duas barras de geração que pode representar, por exemplo, usinas hidroelétricas ou termoelétricas, com seis barras de carga, conforme mostra a Figura 2.1.

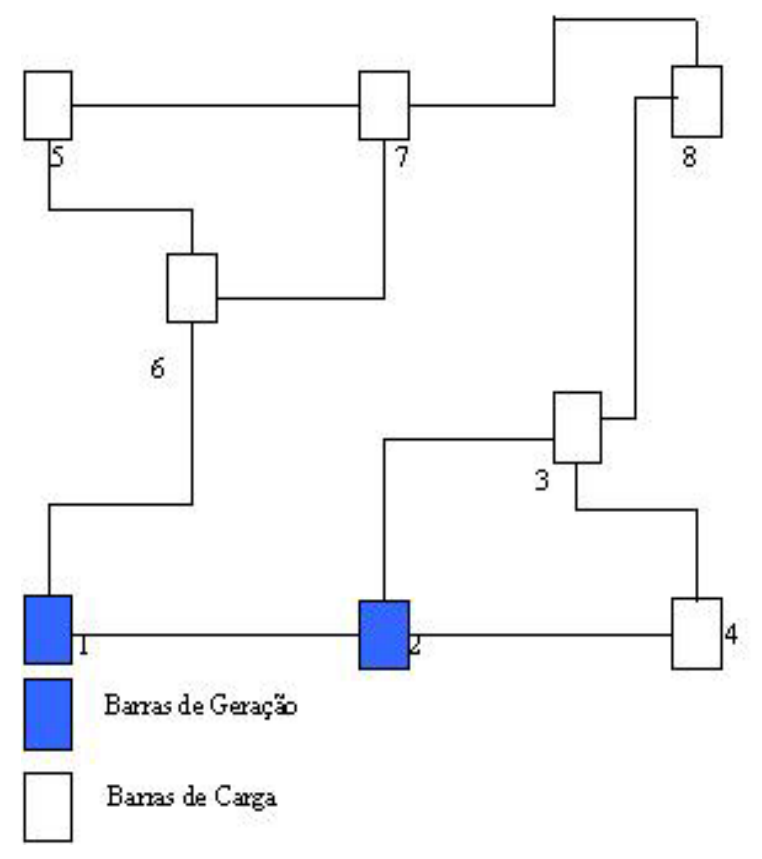

Figura 2.1: Representação de uma rede elétrica

Nesta figura, a potência produzida pelas barras de geração 1 e 2 deverá fluir pelo sistema elétrico para atender às demandas das barras de carga. Assim, é possível estabelecer uma relação entre a Figura 2.1 e o grafo mostrado na Figura 2.2 em que cada nó representa barra de geração ou barra de carga e cada arco representa uma linha de transmissão.

Com base na Figura 2.2 e na lei dos nós de Kirchhoff (ALMEIDA, 87; AZEVEDO, 2002), podemos estabelecer a seguinte relação:

$$
\text { Af }=p-l
$$

em que $A$ corresponde à matriz de incidência nó-arco, $f$ o valor do fluxo de potência em cada linha de transmissão, $p$ a geração de potência em cada barra de geração e $l$ a demanda de potência em cada barra de carga.

A equação (2.6) expressa o balanço de potência nos nós da rede elétrica, isto é, a injeção de potência líquida em cada nó deve ser igual ao fluxo líquido que escoa pelos arcos 


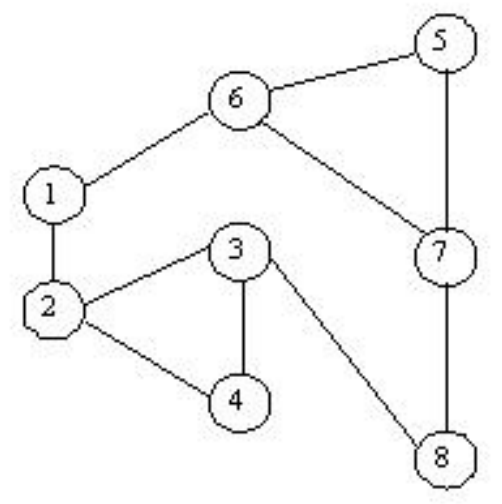

Figura 2.2: Representação em grafo da rede elétrica da figura 2.1

ligados a ele. Além disso, devemos considerar que a soma das tensões em cada percurso fechado existente na rede elétrica deve ser igual a zero, ou seja,

$$
T f=0
$$

em que $T$ representa a matriz de reatância.

A equação (2.7) nada mais é do que a segunda lei de Kirchhoff (MONTICELLI; GARCIA, 1999). A Figura 2.3 ilustra esta situação.

Na descrição do modelo matemático devemos levar em consideração, ainda, os limites de geração e transmissão de energia elétrica, dados por:

$$
\begin{gathered}
p^{\text {min }} \leq p \leq p^{\max } \\
f^{\text {min }} \leq f \leq f^{\text {max }}
\end{gathered}
$$

em que $p^{\min }, p^{\max }$ são os valores mínimo e máximo de $p$, enquanto que $f^{\min }$ e $f^{\max }$, correspondem aos valores mínimo e máximo de $f$, respectivamente.

Finalmente, introduzimos a seguinte função objetivo quadrática com variáveis que determinam os valores ótimos dos fluxos de potência $f$ e dos valores de geração $p$,

$$
\gamma(f, p)=\frac{\alpha}{2}\left[f^{\prime} R f+r^{\prime} f\right]+\frac{\beta}{2}\left[p^{\prime} Q p+c^{\prime} p\right]
$$




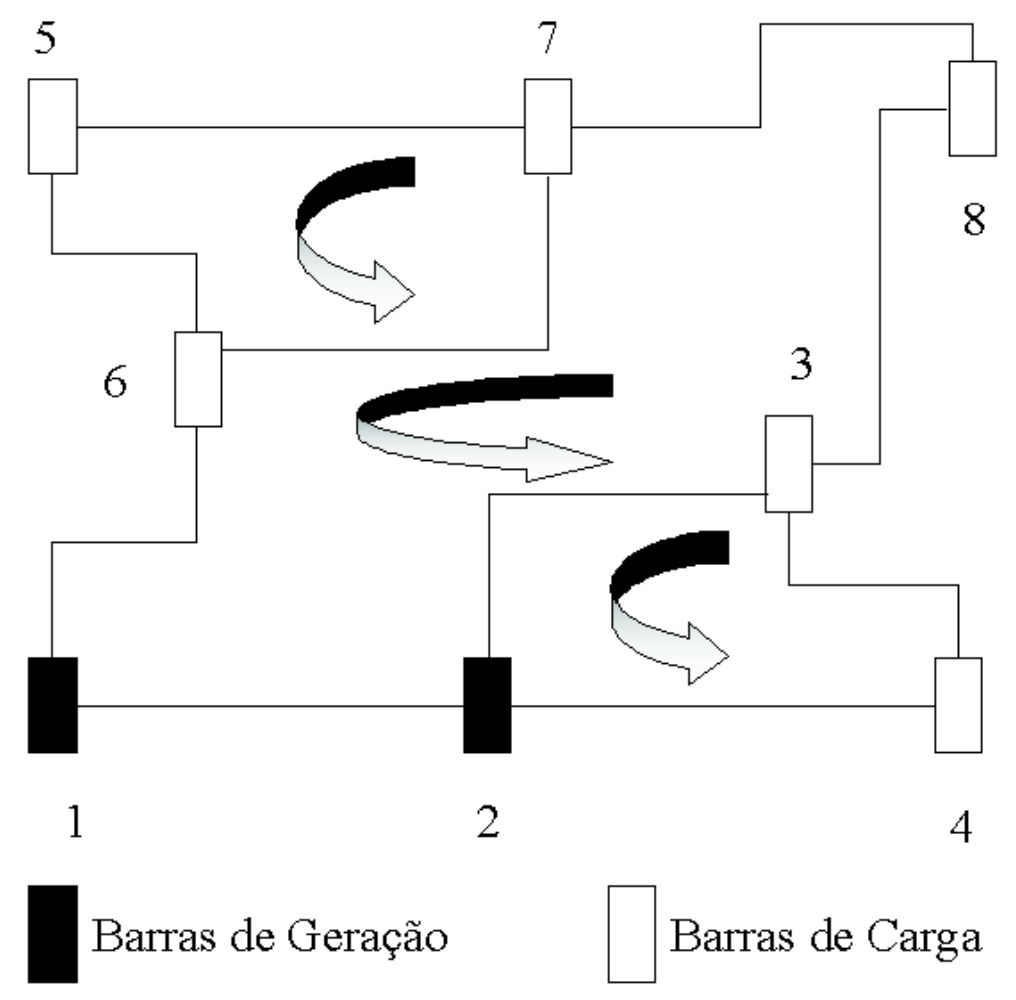

Figura 2.3: Representação da lei das malhas na rede elétrica

em que $\frac{1}{2}\left[f^{\prime} R f+r^{\prime} f\right]$ representa a parcela referente às perdas de transmissão, $R$ a matriz diagonal associada às resistências das linhas de transmissão, $\frac{1}{2}\left[p^{\prime} Q p+c^{\prime} p\right]$ a parcela referente ao custo de geração, $Q$ a matriz diagonal dos coeficientes do termo quadrático de geração, $c$ e $r$ são vetores dos coeficientes lineares e $\alpha$ e $\beta$ são pesos atribuídos à geração e transmissão. Portanto, com esta notação, o modelo matemático estático do fluxo de potência ótimo é dado por:

$$
\begin{array}{cc}
\text { minimizar } & \gamma(f, p) \\
\text { sujeito a } & A f-p=-l \\
& T f=0 \\
& p^{\text {min }} \leq p \leq p^{\text {max }} \\
& f^{\text {min }} \leq f \leq f^{\text {max }}
\end{array}
$$

Se o sistema elétrico em questão estiver ligado a outros sistemas, a troca de potência por meio desta ligação pode ser levada em consideração impondo ao modelo uma restrição de acoplamento. Neste caso, teríamos um modelo dinâmico, como definido na Seção 1.2 


\subsection{O Princípio do Mínimo Esforço}

O princípio do mínimo esforço (PME) consiste em simplificar o modelo de fluxos em redes (1.1) a (1.6) ou (2.10) a (2.14), utilizando a aproximação (2.2). Nesta seção, nos limitaremos a aplicar a referida aproximação ao problema (1.1) a (1.6), cujo modelo é objeto de interesse deste trabalho.

Assim, para cada passo $k$ no tempo $t, k=1,2, \cdots, t$, a equação (2.2) pode ser escrita como:

$$
f^{k}=X^{-1} A^{\prime} \theta^{k}
$$

em que, como visto, $X$ corresponde à matriz diagonal das reatâncias das linhas $\left(X \in R^{n \times n}\right)$ e $\theta^{k}$ o vetor dos ângulos das tensões (MONTICELLI et al., 1982; ALMEIDA, 87).

Substituindo (2.15) em cada termo das Equações (1.1) a (1.4), nas quais aparecem o fluxo $f^{k}$, temos:

$$
\begin{aligned}
\left(f^{k}\right)^{\prime} R f^{k}+r^{\prime} f^{k} & =\left(X^{-1} A^{\prime} \theta^{k}\right)^{\prime} R\left(X^{-1} A^{\prime} \theta^{k}\right)+r^{\prime}\left(X^{-1} A^{\prime} \theta^{k}\right) \\
& =\left(\theta^{k}\right)^{\prime} A\left(X^{-1}\right)^{\prime} R X^{-1} A^{\prime} \theta^{k}+r^{\prime} X^{-1} A^{\prime} \theta^{k} \\
& =\left(\theta^{k}\right)^{\prime} \tilde{B} \theta^{k}+c_{\theta}^{\prime} \theta^{k},
\end{aligned}
$$

em que $\tilde{B}=A\left(X^{-1}\right)^{\prime} R X^{-1} A^{\prime}, U=X^{-1} A^{\prime}$ e $c_{\theta}^{\prime}=r^{\prime} U$.

$$
A f^{k}=A X^{-1} A^{\prime} \theta^{k}=B \theta^{k}
$$

em que $B=A X^{-1} A^{\prime}=A U$.

$$
T f^{k}=T X^{-1} A^{\prime} \theta^{k}=\tilde{A}^{\prime} \theta^{k},
$$

em que $\tilde{A}=T X^{-1} A^{\prime}=T U$.

$$
f^{\min } \leq f^{k} \leq f^{\max } \Longleftrightarrow f^{\min } \leq X^{-1} A^{\prime} \theta^{k} \leq f^{\max } \Longleftrightarrow f^{\min } \leq U \theta^{k} \leq f^{\max }
$$


Com estas notações, o modelo de fluxos em redes (1.1) a (1.6) pode ser reescrito, em termos do princípio do mínimo esforço, na forma:

$$
\begin{array}{cl}
\text { minimizar } \frac{\alpha}{2} \sum_{k=1}^{t}\left[\left(\theta^{k}\right)^{\prime} \tilde{B} \theta^{k}+c_{\theta}^{\prime} \theta^{k}\right]+\frac{\beta}{2} \sum_{k=1}^{t}\left[\left(p^{k}\right)^{\prime} Q p^{k}+c^{\prime} p^{k}\right] & \\
\text { sujeito a } & k=1, \cdots, t \\
B \theta^{k}-E p^{k}=-l^{k}, & k=1, \cdots, t \\
\tilde{A} \theta^{k}=0, & k=1, \cdots, t \\
f^{\text {min }} \leq U \theta^{k} \leq f^{\text {max }}, & k=1, \cdots, t \\
p^{\text {min }} \leq p^{k} \leq p^{\text {max }}, & \\
\sum_{k=1}^{t} p^{k} & =q .
\end{array}
$$

Neste modelo, o vetor dos ângulos das tensões $\theta^{k}$ representa uma variável livre, enquanto que a matriz $B$ (equivalentemente, $\tilde{B}$ ) é uma matriz quadrada de ordem $m \times m$ e só depende dos parâmetros da rede. Para $i, j=1,2, \cdots, m$, ela é definida como:

$$
b_{i j}= \begin{cases}\sum_{l=1}^{m} b_{i l}, & i \neq l \quad \text { e } i=j \\ -b_{i j}, & \text { se } i \neq j \\ 0, & \text { se }(i, j) \notin \Omega\end{cases}
$$

em que $\Omega$ é o conjunto de ramos da rede.

Esta matriz $B$ é conhecida como matriz de susceptância da rede e é uma matriz simétrica e singular.

A questão da singularidade da matriz $B$ pode ser contornada se eliminarmos uma barra $j$ do sistema. Isto é equivalente a eliminar a j-ésima linha e a j-ésima coluna da matriz $B$. É possível mostrar que a matriz resultante de ordem $(m-1) \times(m-1)$ é não singular. Esta demonstração pode ser vista no Apêndice B.

\subsection{Conclusão}

Neste capítulo, estabelecemos um modelo matemático para o problema do fluxo de potência ótimo linearizado DC voltado para a geração e transmissão de sistemas de potência e apresentamos o princípio do mínimo esforço que simplifica o modelo de fluxo em redes. 
Além disso, observamos que a matriz de suceptância da rede é simétrica e singular, mas que esta questão da singularidade pode ser contornada eliminando uma barra do sistema de potência. 


\section{Capítulo 3}

\section{Métodos de Pontos Interiores}

\subsection{Introdução}

O grande interesse em métodos de pontos interiores para problemas de programação linear ocorreu a partir da contribuição dada por Karmarkar (KARMARKAR, 1984) em 1984. Segundo ele, o método de ponto interior proposto resolveria problemas de programação linear de grande porte até 50 vezes mais rápido do que o método Simplex. Isto, de fato, é verdade para algumas classes de problemas.

A idéia básica de pontos interiores é mover-se em direção ao ótimo através do interior da região factível do modelo de programação linear. Esta idéia já havia sido apresentada desde 1967 com o método afim-escala de Dikin (DIKIN, 1967) e o método de Barreira Logaritmica de Fiacco e McCormick (FIACCO; MCCORMICK, 1968). Estes métodos, entretanto, não se mostraram competitivos com o método Simplex, pois exigiam significativamente mais memória que o método Simplex, o que era inaceitável para a época (BEASLEY, 1996).

Neste capítulo, trataremos principalmente dos métodos de pontos interiores primaldual e preditor corretor que, entre as muitas variantes dos métodos de pontos interiores, são os mais elegantes teoricamente e os de melhor desempenho computacional para problemas de programação linear. Antes de introduzir estes métodos, apresentaremos algumas informações gerais sobre otimização, condições de otimalidade, multiplicadores de Lagrange e barreira logarítmica. 


\subsection{O Problema da Otimização}

No campo da otimização busca-se a formulação e solução de problemas no sentido de otimizar algum critério de decisão. Em geral, as decisões a serem tomadas estão sujeitas a restrições, pois, nem todas as decisões possíveis são aceitáveis.

Resolver um problema de otimização do ponto de vista matemático requer que ambas, a função objetivo e as restrições, sejam quantificadas. Isto pode ser difícil, pois se importantes aspectos não puderem ser quantificados, eles não poderão ser incluídos no modelo matemático e a solução para o problema de otimização formulado matematicamente pode ter pouco ou quase nada a ver com o problema real. Assim, é fundamental verificar se a solução obtida por meios matemáticos faz sentido quando aplicada ao problema real.

É possível formular o mesmo problema utilizando diferentes modelos matemáticos. A escolha do modelo apropriado é importante, pois a dificuldade de resolver o problema matematicamente depende, de forma dramática, da categoria na qual o modelo está inserido. Por exemplo, a classe de métodos de pontos interiores pode ser aplicada para resolver problemas cujas decisões são quantidades contínuas e com restrições de desigualdade (SPORRE, 2003).

\subsubsection{Diferentes Formas de Otimização}

Um problema de otimização em sua forma mais geral é definido por:

$$
\text { minimizar } f(\mathbf{x}), \quad \mathbf{x} \in \mathbf{F}
$$

em que $F$ é a região factível do problema e tipicamente é um subconjunto de algum conjunto $\tilde{F}$ mais geral. Por exemplo, $F$ pode ser o espaço Euclidiano n-dimensional, o espaço de todas as funções que são quadrado integráveis no intervalo [0,1], ou seja, pertencentes ao espaço $L_{2}[0,1]$ ou o conjunto de todas as matrizes reais simétricas de ordem n. A função objetivo $f(\mathbf{x})$ associa um valor real a cada elemento de $F$, isto é,

$$
f: F \rightarrow R
$$

Ao resolver um problema de otimização, a idéia consiste em calcular um ponto $\mathbf{x}^{*}$ que representa um conjunto de decisões, tal que $f\left(\mathbf{x}^{*}\right) \leq \mathbf{f}(\mathbf{x}), \forall \mathbf{x} \in \mathbf{F}$. Este ponto é chamado de mínimo global para (3.1). 
O conjunto $F$ pode também ser de dimensão infinita. Entretanto, a menos que uma solução analítica possa ser obtida, problemas desta natureza são muito difíceis de serem resolvidos. Na verdade, o problema (3.1) é muito geral para permitir a construção de métodos práticos, mesmo se $F$ for de dimensão finita (SPORRE, 2003).

\subsubsection{Programação Linear}

Na classe de problemas de programação linear o conjunto $F \subset R^{n}$ para algum inteiro positivo n é definido por uma quantidade de restrições de igualdade e desigualdade lineares, enquanto que a função objetivo $f(\mathbf{x})$ é linear.

Assim, um problema de programação linear típico consiste em:

$$
\begin{array}{cc}
\operatorname{minimizar} & f(\mathbf{x})= \\
\text { sujeito a } & \mathbf{c}^{\prime} \mathbf{x}, \quad \mathbf{x} \in \mathbf{R}^{\mathbf{n}} \\
& A \mathbf{x}=\mathbf{b} \\
& \mathbf{x} \geq \mathbf{0}
\end{array}
$$

em que $A$ é uma matriz de dimensão $m \times n$, b é um vetor de dimensão $m \times 1$ e $\mathbf{c}$ é um vetor de dimensão $n \times 1$.

A forma como está apresentado o problema (3.2) é conhecida como forma padrão (SPORRE, 2003). Como qualquer problema de programação linear pode ser convertido para a forma padrão, não existe perda de generalidade em limitar o estudo dos problemas a esta forma.

Para cada problema de programação linear existe um problema dual associado, que por sua vez é também um problema de programação linear. O dual associado a (3.2) é definido por:

$$
\begin{array}{cc}
\operatorname{maximizar} & g(\mathbf{y})=\mathbf{b}^{\prime} \mathbf{y}, \quad \mathbf{y} \in \mathbf{R}^{\mathbf{m}} \\
\text { sujeito a } & A^{\prime} \mathbf{y}+\mathbf{z}=\mathbf{c}, \quad \mathbf{z} \in \mathbf{R}^{\mathbf{n}} \\
\mathbf{z} \geq \mathbf{0}
\end{array}
$$

O problema (3.2) é chamado de problema primal. O par $(\mathbf{y}, \mathbf{z})$ é conhecido como dual factível se $\mathbf{z}$ é não negativo e $A^{\prime} \mathbf{y}+\mathbf{z}=\mathbf{c}$. Analogamente, $\mathbf{x} \geq \mathbf{0}$ satisfazendo $A \mathbf{x}=\mathbf{b}$ é conhecido como primal factível. 
Um resultado fundamental que relaciona os problemas (3.2) e (3.3) é que se um dos problemas tem uma solução ótima, então, o outro também tem e os valores das funções objetivos nestes ótimos são iguais. Tal resultado é conhecido como o teorema da dualidade forte de programação linear.

\subsubsection{Programação Quadrática}

A programação quadrática é uma extensão natural de programação linear. De fato, a região factível $F$ é da mesma forma (poliedral) que para programação linear, mas a função objetivo $f(\mathbf{x})$ deixa de ser linear para assumir a forma quadrática, isto é,

$$
f(\mathbf{x})=\frac{1}{2} \mathbf{x}^{\prime} \mathbf{Q} \mathbf{x}+\mathbf{c}^{\prime} \mathbf{x}
$$

em que $Q$ é uma matriz quadrada de ordem $n$.

Observemos que se $Q=0, f(\mathbf{x})$ é linear, mostrando que programação linear é um caso particular de programação quadrática.

Um complicador que surge em programação quadrática geral quando comparada com programação linear é a presença de mínimos locais que não são mínimos globais.

Definição 3.1 Um ponto $\mathbf{x}^{*}$ é um mínimo local de $f(\mathbf{x})$ sobre $\tilde{F}$ se não existe localmente nas proximidades de $x^{*}$ um ponto melhor em $\tilde{F}$, ou seja, se existe um escalar $\epsilon>0$ tal

que $f\left(\mathbf{x}^{*}\right) \leq \mathbf{f}(\mathbf{x}), \forall \mathbf{x} \in \tilde{\mathbf{F}} \cap \mathbf{B}\left(\mathbf{x}^{*}, \epsilon\right)$, em que $B\left(x^{*}, \epsilon\right)=\left\{\mathbf{x}:\left\|\mathbf{x}-\mathbf{x}^{*}\right\|<\epsilon\right\}$ é a bola aberta de raio $\epsilon$ em torno de $\mathbf{x}^{*}$.

Um problema de programação quadrática geral é muito difícil de resolver, entretanto, existe uma subclasse conhecida como programação quadrática convexa para a qual é possível calcular um mínimo global de forma eficiente (SPORRE, 2003).

\subsubsection{Programação Quadrática Convexa}

Definição 3.2 Um conjunto $C$ é convexo se o segmento de reta que liga qualquer par de pontos no conjunto está contido inteiramente no conjunto, ou seja, se $\alpha x+(1-\alpha) y \in C$ para todo $x, y \in C$ e $\alpha \in[0,1]$. 
Definição 3.3 Uma função $f(x)$ definida sobre um conjunto convexo $C$ é uma função convexa se $f(x+(1-\alpha) y) \leq \alpha f(x)+(1-\alpha) f(y), \forall x, y \in C$ e $\alpha \in[0,1]$. Ou seja, se o interpolador linear entre $(x, f(x)) e(y, f(y))$ não repousar abaixo de $(z, f(z))$, para algum $z$ pertencente ao segmento de reta que liga $x$ e $y$.

Uma implicação do conceito de convexidade é que qualquer mínimo local é também um mínimo global. Além disto, calcular um mínimo global para problemas convexos é uma tarefa factível do ponto de vista computacional (SPORRE, 2003).

Em programação quadrática e linear a região factível $F$ é poliedral, ou seja, é um conjunto convexo. Se restringirmos $f(x)$ a ser não apenas quadrática, mas também convexa obtemos a subclasse constituída pelos problemas de programação quadrática convexa.

De maneira geral, um problema de programação quadrática convexa pode ser posto na seguinte forma:

$$
\begin{array}{cc}
\operatorname{minimizar} & f(\mathbf{x})=\frac{\mathbf{1}}{2} \mathbf{x}^{\prime} \mathbf{Q} \mathbf{x}+\mathbf{c}^{\prime} \mathbf{x}, \quad \mathbf{x} \in \mathbf{R}^{\mathbf{n}} \\
\text { sujeito a } & A \mathbf{x}=\mathbf{b} \\
\mathbf{x} \geq \mathbf{0}
\end{array}
$$

em que $Q$ é simétrica e semi definida positiva.

\subsection{Multiplicadores de Lagrange}

Nesta Seção, analisaremos o problema geral de minimizar uma função $f(x)$ sujeita a uma ou mais restrições de igualdade. A única exigência sobre $f(x)$ é que ela seja, no mínimo, duas vezes diferenciável. Consideremos, então, o seguinte problema de otimização:

$$
\begin{array}{cc}
\text { minimizar } & f(x) \\
\text { sujeito a } & g(x)=0 \\
& x \geq 0
\end{array}
$$

em que $g(x)=0$ corresponde a um conjunto de $m$ restrições.

O gradiente de $f(x)$, denotado por $\nabla f$, é um vetor que aponta na direção de maior crescimento de $f(x)$. Se o problema de otimização fosse irrestrito bastaria fazermos 
$\nabla f(x)=0$ para determinarmos os pontos críticos de $f(x)$ e, o mínimo, se existisse, deveria pertencer a este conjunto (de pontos críticos). Para o caso particular em que $m=1$, devido à presença da restrição isolada $g(x)=0$, o gradiente de $g(x)$ deve ser ortogonal ao conjunto de soluções factíveis $F=\{x / g(x)=0\}$, ou seja, $\nabla g(x) \cdot F=0$, para cada $x \in F$. Assim, para que um ponto $x^{*}$ seja um ponto crítico é necessário que ele pertença ao conjunto factível $F, g\left(x^{*}\right)=0$ e que $\nabla f\left(x^{*}\right)$ seja proporcional a $\nabla g\left(x^{*}\right)$, ou seja,

$$
\begin{aligned}
g\left(x^{*}\right) & =0 \\
\nabla f\left(x^{*}\right) & =\lambda \nabla g\left(x^{*}\right)
\end{aligned}
$$

em que $\lambda \in R$ é a constante de proporcionalidade conhecida como multiplicador de Lagrange.

No caso em que $m>1$ a região de factibilidade $F$ é a intersecção de $m$ hiperplanos e o sistema constituído pelas equações (3.7) e (3.8) deve ser reescrito como:

$$
\begin{aligned}
g\left(x^{*}\right) & =0 \\
\nabla f\left(x^{*}\right) & =\sum_{i=1}^{m} \lambda_{i} \nabla g\left(x^{*}\right) .
\end{aligned}
$$

Em termos formais é possível obter o sistema de equações anteriores introduzindo a seguinte função, conhecida como função Lagrangeana:

$$
L(x, \lambda)=f(x)-\sum_{i=1}^{m} \lambda_{i} g_{i}(x) .
$$

Derivando (3.11) parcialmente com relação a x e $\lambda_{i}$ e igualando a zero, temos:

$$
\begin{aligned}
\frac{\partial L}{\partial x_{j}} & =\frac{\partial f}{\partial x_{j}}-\sum_{i=1}^{m} \lambda_{i} \frac{\partial g_{i}}{\partial x_{j}}=0, \quad j=1,2, \ldots, n \\
\frac{\partial L}{\partial \lambda_{i}} & =-g_{i}(x)=0, \quad i=1,2, \ldots, m .
\end{aligned}
$$

Em notação vetorial observemos que as equações (3.12) e (3.13) correspondem exatamente às equações (3.9) e (3.10). Estas equações são conhecidas como condições de otimalidade de primeira ordem. Determinar se uma solução das equações (3.12) e (3.13) é um mínimo global do problema (3.6) pode ser algo muito difícil. Todavia, se as restrições forem todas lineares, o Teorema 3.1, enunciado a seguir e cuja demonstração análoga para o máximo 
local encontra-se em (VANDERBEI, 1996), indica um meio de calcular um mínimo local de $f(x)$.

Teorema 3.1 Se o conjunto de restrições for linear, um ponto crítico $x^{*}$ é um mímimo local se

$$
\xi^{\prime} H f\left(x^{*}\right) \xi>0
$$

para todo $\xi \neq 0$ satisfazendo

$$
\xi^{\prime} \nabla g_{i}\left(x^{*}\right)=0, \quad i=1,2, \ldots, m
$$

em que

$$
H f\left(x^{*}\right)=\left[\frac{\partial^{2} f}{\partial x_{i}^{*} \partial x_{j}^{*}}\right]
$$

é a matriz Hessiana de $f$ no ponto $x^{*}$.

\subsection{O Problema da Barreira Logarítmica}

Métodos de pontos interiores são intimamente relacionados com métodos de barreira, que foram desenvolvidos por Fiacco e McCormick no final da década de 60 (JENSEN; BARD, ). A idéia do método está baseada na resolução aproximada de uma sequência de subproblemas sem qualquer restrição de desigualdade. Sob certas condições, a sequência de soluções aproximadas converge para a solução do problema original.

Consideremos o seguinte problema de maximização com restrição:

$$
\begin{array}{cc}
\operatorname{maximizar} & f(x)=c^{\prime} x \\
\text { sujeito a } & A x+w=b \\
& x, w \geq 0 .
\end{array}
$$

Neste problema, podemos remover a restrição de não negatividade para cada variável adicionando-a à função objetivo $f(x)$ uma função que é escolhida de tal forma que se aproxima do infinito quando a variável em questão aproxima-se de zero. A função mais simples com esta propriedade é o logaritmo. Portanto, para cada variável, introduzimos um novo termo na função objetivo que corresponde a uma constante multiplicada pelo 
logaritmo da variável, ou seja:

$$
\begin{array}{cc}
\operatorname{maximizar} & f(x)+\mu \sum_{j} \log x_{j}+\mu \sum_{i} \log w_{i} \\
\text { sujeito a } & A x+w=b
\end{array}
$$

em que o parâmetro $\mu$ é maior que zero.

O problema (3.18) é chamado de problema barreira logaritmica associado com (3.17). Observemos que ele não é realmente um problema , mas uma família de problemas associada ao parâmetro $\mu$. Cada um destes problemas é um problema de programação não linear porque a função objetivo é não linear. Esta função não linear é conhecida como função barreira logarítmica.

Se o problema original for um problema de programação linear, então, a região factível $F$ é um poliedro, com cada face sendo caracterizada pela propriedade que, nela, cada uma das variáveis é nula. Portanto, a função barreira tende a menos infinito sobre cada face do poliedro. Além disto, ela é finita no interior do poliedro e se aproxima de menos infinito nas proximidades do contorno. Para cada $\mu>0$, o máximo é atingido em um ponto interior e quando $\mu$ está próximo de zero este ponto interior move-se na direção da solução ótima do problema original. Visto como uma função de $\mu$ o conjunto de soluções ótimas para o problema da barreira forma um caminho através do interior do poliedro que representa o conjunto das soluções factíveis. Este caminho é denominado caminho central. O objetivo das próximas seções é estudar este caminho central (VANDERBEI, 1996).

No que segue, mostraremos que para cada valor do parâmetro barreira $\mu$ existe uma única solução para o problema da barreira. Além disso, se $\mu \rightarrow 0$, a solução do problema tende para a solução do problema de programação linear original. O problema (3.18) é um problema de otimização com restrição de igualdade e assim, podemos associar a ele o seu Lagrangeano, ou seja,

$$
L(x, w, \lambda)=c^{t} x+\mu \sum_{j} \log x_{j}+\mu \sum_{i} \log w_{i}+\lambda^{t}(b-A x-w)
$$

Derivando (3.19) parcialmente com relação a cada variável, obtemos as seguintes condições de otimalidade: 


$$
\begin{aligned}
\frac{\partial L}{\partial x_{j}}=c_{j}+\mu \frac{1}{x_{j}}-\sum_{i} \lambda_{i} a_{i j} & =0, \quad j=1,2, \ldots, n \\
\frac{\partial L}{\partial w_{i}}=\mu \frac{1}{w_{i}}-\lambda_{i} & =0, \quad i=1,2, \ldots, m \\
\frac{\partial L}{\partial \lambda_{i}}=b_{i}-\sum_{j} a_{i j} x_{j}-w_{i} & =0, \quad i=1,2, \ldots, m .
\end{aligned}
$$

Na forma matricial, estas condições de otimalidade são dadas por

$$
\begin{aligned}
A^{\prime} \lambda-\mu X^{-1} e & =c \\
\lambda & =\mu W^{-1} e \\
A x+w & =b
\end{aligned}
$$

em que $X$ e $W$ são matrizes diagonais cujos elementos são, respectivamente, as componentes dos vetores $x$ e $w$ e $e$ denota o vetor que possui todos os elementos iguais a 1.

Definindo $z=\mu X^{-1} e$ (EL-BAKRY et al., 1996), as condições de otimalidade (3.20) podem ser reescritas como:

$$
\begin{aligned}
A x+w & =b \\
A^{\prime} \lambda-z & =c \\
z & =\mu X^{-1} e \\
\lambda & =\mu W^{-1} e .
\end{aligned}
$$

Multiplicando a terceira e quarta equações, respectivamente, por $X$ e $W$, obtemos finalmente a seguinte forma simétrica primal-dual das condições de otimalidade de primeira ordem:

$$
\begin{aligned}
A x+w & =b \\
A^{\prime} \lambda-z & =c \\
X Z e & =\mu e \\
\Lambda W e & =\mu e .
\end{aligned}
$$




\subsection{A Existência e Unicidade de Solução para o Pro- blema da Barreira}

Uma solução para o problema da barreira nem sempre existe. O Teorema 3.2 a seguir, cuja demonstração pode ser encontrada em (VANDERBEI, 1996), fornece uma condição necessária e suficiente para a existência de solução para este problema.

Teorema 3.2 Uma solução para o problema da barreira existe se, e somente se, as regiões factíveis para o primal e dual têm interior não vazio.

Para mostrar que se uma solução existe ela deve ser única, devemos analisar o comportamento da Hessiana da função barreira

$$
f(x, w)=c^{\prime} x+\mu \sum_{j} \log x_{j}+\mu \sum_{i} \log w_{i}
$$

que é dada por:

$$
H=\left[\begin{array}{rr}
-\frac{\mu}{x_{j}^{2}} & 0 \\
0 & -\frac{\mu}{w_{i}^{2}}
\end{array}\right], i=1,2, \ldots, m, \quad j=1,2, \ldots, m .
$$

Observemos que $H$ é diagonal com elementos estritamente negativos. Portanto, pelo Teorema 3.1 existe no máximo um ponto crítico e, se existe, é um máximo global.

O Corolário 3.1 a seguir resume estes resultados:

Corolário 3.1 Se um conjunto factível primal (ou seu dual) possui um interior não vazio e é limitado, então para cada $\mu>0$ existe uma única solução $\left(x_{\mu}, w_{\mu}, \lambda_{\mu}, z_{\mu}\right)$ para as condições de otimalidade de primeira ordem definidas em (3.21).

O caminho $\left\{\left(x_{\mu}, w_{\mu}, \lambda_{\mu}, z_{\mu}\right) / \mu>0\right\}$ é chamado caminho central primal-dual e desempenha um papel importante em métodos de pontos interiores para programação linear.

\subsection{Condições de Otimalidade para os Problemas Pri- mal e Dual}

Por conveniência, vamos reescrever o problema primal (3.2) e o problema dual associado (3.3). 


$$
\begin{array}{rlrl}
\operatorname{minimizar} & f(x) & =c^{\prime} x \\
\text { sujeito a } & A x & =b \\
& x \geq 0
\end{array}
$$

$$
\begin{array}{cc}
\operatorname{maximizar} & g(y)=b^{\prime} y \\
\text { sujeito a } & A^{\prime} y+z=c \\
& z \geq 0 .
\end{array}
$$

A teoria da dualidade explica a relação entre os dois problemas (3.22) e (3.23). De fato, o conjunto factível e o conjunto solução para o primal informam-nos um pouco sobre o dual e, reciprocamente. Por exemplo, dado qualquer vetor factível $x$ para $(3.22)$ e $(y, z)$ para (3.23), temos que

$$
g(y) \leq f(x)
$$

Em outras palavras, a função objetivo do dual fornece um limite inferior para a função objetivo do primal. As duas funções objetivos coincidirão para as soluções dos problemas, isto é, $g\left(y^{*}\right)=f\left(x^{*}\right)$, em que $x^{*}$ resolve o problema $(3.22)$ e $\left(y^{*}, z^{*}\right)$ resolve o problema (3.23).

As condições de otimalidade para (3.22) e (3.23) podem ser estabelecidas como casos particulares das condições de otimalidade para problemas gerais de otimização com restrição, conhecidas como condições de Karush-Kuhn-Tucker (KKT).

Podemos enunciar, então, as condições de otimalidade, como segue (WRIGHT, 1997):

O vetor $x^{*} \in R^{n}$ é uma solução de (3.22) se, e somente se, existem vetores $z^{*} \in R^{n}$ e $y^{*} \in R^{m}$ para os quais as seguintes condições são satisfeitas para $(x, y, z)=\left(x^{*}, y^{*}, z^{*}\right)$ : 


$$
\begin{aligned}
A^{\prime} y+z & =c \\
A x & =b \\
x_{i} z_{i} & =0, \quad i=1,2, \ldots, n, \\
(x, z) & \geq 0 .
\end{aligned}
$$

Por analogia com o problema geral de otimização com restrição, os vetores $y$ e z são multiplicadores de Lagrange para as restrições $A x=b$ e $x \geq 0$, respectivamente.

A condição (3.27) implica que para cada $i=1,2, \ldots, n$, uma das componentes $x_{i}$ ou $z_{i}$ deve ser zero. Esta condição é conhecida como condição de complementaridade.

Analisando as condições (3.25) a (3.28) do ponto de vista primal e dual, concluímos que um vetor $\left(x^{*}, y^{*}, z^{*}\right)$ resolve este sistema se, e somente se, $x^{*}$ resolve o problema primal (3.22) e $\left(y^{*}, z^{*}\right)$ resolve o problema dual (3.23). O vetor $\left(x^{*}, y^{*}, z^{*}\right)$ é chamado de solução primal-dual.

Os métodos de pontos interiores primais-duais que veremos a seguir, são obtidos por meio da aplicação do método de Newton (Apêndice A) a estas condições de otimalidade.

\subsection{Métodos Primais-Duais}

Vamos reescrever as condições de otimalidade definidas em (3.25) a (3.28) em uma forma ligeiramente diferente, por meio da aplicação $F: R^{2 n+m} \rightarrow R^{2 n+m}$ :

$$
\begin{gathered}
F(x, y, z)=\left[\begin{array}{r}
A^{\prime} y+z-c \\
A x-b \\
X Z e
\end{array}\right]=0 \\
(x, z) \geq 0
\end{gathered}
$$

em que

$$
X=\operatorname{diag}\left(x_{1}, x_{2}, \ldots, x_{n}\right), Z=\operatorname{diag}\left(z_{1}, z_{2}, \ldots, z_{n}\right) \text { e } e=(1,1, \ldots, 1)^{\prime} \text {. Observemos que }
$$

$F$ é linear com relação aos termos $A^{\prime} y+z-c$ e $A x-b$, permanecendo não linear o termo remanescente $X Z e$. 
Todos os métodos primais-duais geram uma seqüência $\left(x^{k}, y^{k}, z^{k}\right)$ que satisfaz, para cada iteração $k$, a desigualdade (3.29) estritamente, isto é, $x^{k}>0$ e $z^{k}>0$. Esta propriedade é que dá origem ao termo ponto interior (WRIGHT, 1997). Muitos métodos de pontos interiores exigem que a seqüência $\left(x^{k}, y^{k}, z^{k}\right)$ seja estritamente factível, ou seja, cada $\left(x^{k}, y^{k}, z^{k}\right)$ deve satisfazer as restrições de igualdade tanto para o problema primal quanto para o problema dual. Neste sentido, definimos, respectivamente, os espaços $S$ e $S^{0}$ de todas as soluções factíveis e estritamente factíveis, por:

$$
\begin{aligned}
S & =\left\{(x, y, z) / A x=b, \quad A^{\prime} y+z=c, \quad(x, z) \geq 0\right\} \\
S^{0} & =\left\{(x, y, z) / A x=b, \quad A^{\prime} y+z=c, \quad(x, z)>0\right\} .
\end{aligned}
$$

Os métodos primais-duais apresentam dois aspectos básicos em suas estruturas: um procedimento para determinar o passo e uma medida que define o grau de aceitação de cada ponto no espaço de pesquisa. O procedimento que fornece a direção de busca tem sua origem no método de Newton. Este constitui um modelo linear para a aplicação $F$ em torno do ponto atual e fornece a direção de busca $\left(\Delta x^{k}, \Delta y^{k}, \Delta z^{k}\right)$ por meio da solução do seguinte sistema de equações lineares:

$$
J\left(x^{k}, y^{k}, z^{k}\right)\left[\begin{array}{c}
\Delta x^{k} \\
\Delta y^{k} \\
\Delta z^{k}
\end{array}\right]=-F\left(x^{k}, y^{k}, z^{k}\right),
$$

em que $J$ é o Jacobiano de $F$. Se o ponto $\left(x^{k}, y^{k}, z^{k}\right) \in S^{0}$, as equações que definem o passo de Newton tornam-se

$$
\left[\begin{array}{rrr}
0 & A^{\prime} & I \\
A & 0 & 0 \\
Z & 0 & X
\end{array}\right]\left[\begin{array}{c}
\Delta x^{k} \\
\Delta y^{k} \\
\Delta z^{k}
\end{array}\right]=\left[\begin{array}{r}
0 \\
0 \\
-X Z e
\end{array}\right]
$$

Definimos, então, o novo ponto $\left(x^{k+1}, y^{k+1}, z^{k+1}\right)$, como segue:

$$
\left(x^{k+1}, y^{k+1}, z^{k+1}\right)=\left(x^{k}, y^{k}, z^{k}\right)+\alpha_{k}\left(\Delta x^{k}, \Delta y^{k}, \Delta z^{k}\right)
$$

em que o passo $\alpha_{k}$ é escolhido de tal forma que garanta que $\left(x^{k+1}, z^{k+1}\right)>0$. Isto pode ser feito tomando $\alpha_{k}$ no intervalo $(0,1]$. Este procedimento de Newton defi- 
nido pelo sistema acima não permite que se faça grandes progressos em direção à solução do problema. Os métodos primais-duais modificam este procedimento básico de Newton, aplicando-o não ao sistema definido anteriormente por $F(x, y, z)$, mas a uma versão perturbada deste sistema, conforme veremos a seguir.

\subsubsection{O Caminho Central}

O caminho central $C$ é um arco constituído por pontos estritamente factíveis, isto é, pertencentes a $S^{0}$, que desempenha um papel fundamental na teoria dos métodos primaisduais. Ele é parametrizado por um escalar $\tau>0$, e cada ponto $\left(x_{\tau}, y_{\tau}, z_{\tau}\right) \in C$ resolve o seguinte sistema (WRIGHT, 1997):

$$
\begin{aligned}
A^{\prime} y+z & =c \\
A x & =b \\
x_{i} z_{i} & =\tau, \quad i=1,2, \ldots, n, \\
(x, z) & >0 .
\end{aligned}
$$

Podemos, então, definir o caminho central como

$$
C=\left\{\left(x_{\tau}, y_{\tau}, z_{\tau}\right) / \tau>0\right\}
$$

As Equações (3.32) a (3.35) aproximam as condições de otimalidade definidas em (3.25) a (3.28), à medida que $\tau$ tende a zero. Assim, se $\tau \rightarrow 0, C$ deve convergir para uma solução primal-dual do problema linear. Portanto, o caminho central guia-nos para uma solução por meio de uma rota que é livre de soluções espúrias e mantém cada par $x_{i} z_{i}$ estritamente positivo e decaindo para zero a uma mesma taxa. A vantagem em se tomar passos de Newton em direção a pontos sobre o caminho central $C$, ao invés de passos puros de Newton para $F$, é que usualmente é possível obter passos maiores sem violar a condição de positividade de $(x, z)$. A fim de descrever a direção de pesquisa enviesada para o caminho central, introduzimos um parâmetro de centro $\sigma \in[0,1]$ e uma medida de dualidade $\mu$ definida por 


$$
\mu=\frac{1}{n} \sum_{i=1}^{n} x_{i} z_{i}=\frac{x^{\prime} z}{n},
$$

que corresponde a um valor médio dos produtos $x_{i} z_{i}$. Com este entendimento o sistema

$$
\left[\begin{array}{rrr}
0 & A^{\prime} & I \\
A & 0 & 0 \\
Z & 0 & X
\end{array}\right]\left[\begin{array}{c}
\Delta x^{k} \\
\Delta y^{k} \\
\Delta z^{k}
\end{array}\right]=\left[\begin{array}{r}
0 \\
0 \\
-X Z e
\end{array}\right]
$$

pode ser reescrito como

$$
\left[\begin{array}{rrr}
0 & A^{\prime} & I \\
A & 0 & 0 \\
Z & 0 & X
\end{array}\right]\left[\begin{array}{c}
\Delta x^{k} \\
\Delta y^{k} \\
\Delta z^{k}
\end{array}\right]=\left[\begin{array}{r}
0 \\
0 \\
-X Z e+\sigma_{k} \mu_{k} e
\end{array}\right] .
$$

Se $\sigma_{k}=1$, as equações acima definem uma direção de centragem, um passo de Newton em direção ao ponto $\left(x_{\mu}, y_{\mu}, z_{\mu}\right) \in C$, para o qual todos os produtos $x_{i} z_{i}$ são idênticos a $\mu_{k}$. As direções de centragem, usualmente são enviesadas em direção ao interior do espaço $S^{0}$ e fazem pouco progresso no sentido de reduzir $\mu_{k}$. Entretanto, se elas se movem próximas a $C$, podem favorecer um progresso substancial na redução de $\mu_{k}$, na próxima iteração. De fato, se a próxima iteração inicia nas proximidades de $C$, será possível tomar um passo relativamente grande sem sair do espaço $S^{0}$. Para o caso extremo em que $\sigma^{k}=0$, obtemos o passo padrão de Newton definido no sistema

$$
\left[\begin{array}{rrr}
0 & A^{\prime} & I \\
A & 0 & 0 \\
Z & 0 & X
\end{array}\right]\left[\begin{array}{c}
\Delta x^{k} \\
\Delta y^{k} \\
\Delta z^{k}
\end{array}\right]=\left[\begin{array}{r}
0 \\
0 \\
-X Z e
\end{array}\right]
$$

conhecido também como direção afim escala. Muitos métodos utilizam valores intermediários de $\sigma_{k} \in(0,1)$ com a dupla finalidade de reduzir $\mu_{k}$ e aperfeiçoar a centralidade (WRIGHT, 1997).

\subsubsection{Métodos de Trajetória Central}

Os métodos de trajetória central restringem a seqüência de pontos $\left(x^{k}, y^{k}, z^{k}\right)$ a uma vizinhança do caminho central $C$ e seguem este caminho em busca de uma solução para 
o problema linear.

A vizinhança exclui aqueles pontos $\left(x^{k}, z^{k}\right)$ que estão próximos à fronteira do espaço $S$ das soluções factíveis. Isto implica que as direções de pesquisa calculadas a partir de qualquer ponto pertencente à vizinhança fazem pequenos progressos na direção do conjunto solução (WRIGHT, 1997).

Nos métodos de trajetória central, a medida de dualidade $\mu_{k}$ é forçada a zero quando $k \rightarrow \infty$, de tal modo que a seqüência $\left(x^{k}, y^{k}, z^{k}\right)$ converge para um ponto que satisfaz as condições de KKT (3.25) a (3.28).

As duas vizinhanças mais interessantes de $C$ são as chamadas vizinhança norma 2 $N_{2}(\xi)$ e vizinhança lateral norma - $\infty$, definidas, respectivamente, por (WRIGHT, 1997):

$$
\begin{aligned}
N_{2}(\xi) & =\left\{(x, y, z) \in S^{0} /\|X Z e-\mu e\| \leq \xi \mu\right\}, \\
N_{-\infty}(\xi) & =\left\{(x, y, z) \in S^{0} / x_{i} z_{i} \geq \xi \mu, \forall i=1,2, \ldots, n\right\},
\end{aligned}
$$

para $\xi \in(0,1)$.

Mantendo a seqüência de pontos dentro de uma ou outra destas vizinhanças, os métodos de trajetória central reduzem todos os produtos $x_{i} z_{i}$ a zero, aproximadamente à mesma taxa.

O motivo pelo qual a sequiência de pontos $\left(x^{k}, y^{k}, z^{k}\right)$ desvia do caminho central (permanecem em uma vizinhança deste caminho) é porque, em geral, os produtos $x_{i} z_{i}$ não são idênticos, de tal modo que a condição (3.34) não é satisfeita exatamente. Isto sugere o cálculo de uma medida, conhecida como medida de proximidade, definida por (WRIGHT, July, 1999):

$$
\delta(\mu)=\left\|\frac{X Z e}{\mu}-e\right\|
$$

em que $\|$.$\| tanto pode corresponder à norma 2$ ou à norma- $\infty$. Em ambas as normas é possível assegurar que $x$ e $z$ são estritamente positivos exigindo que $\delta(\mu)<1$.

Se utilizarmos a norma 2 em (3.38) e forçando $\delta(\mu)$ a ser menor ou igual a uma constante $\xi \in[0,1]$, obtemos a vizinhança $N_{2}(\xi)$ definida em (3.36).

Observando que o objetivo principal é fazer com que os produtos $x_{i} z_{i}$ sejam mantidos maiores do que o valor médio $\mu$, podemos estabelecer a vizinhança $N_{-\infty}(\xi)$ já definida 
em (3.37). Com isto, evitamos que $x$ e $z$ se aproximem prematuramente da fronteira da região $(x, z) \geq 0$. Portanto, a vizinhança $N_{-\infty}(\xi)$ utiliza apenas uma região em relação a $x_{i} z_{i}$, em lugar de duas como ocorre em (3.36).

Assim, métodos de trajetória central selecionam uma vizinhança $\left(N_{2}\right.$ ou $\left.N_{-\infty}\right)$ e escolhem o parâmetro de centragem $\sigma$ e o passo $\alpha$ para garantir que cada termo da seqüência $\left(x^{k}, y^{k}, z^{k}\right)$ permaneça dentro da vizinhança escolhida.

A escolha do parâmetro $\sigma$ desempenha um papel importante tanto na teoria quanto na prática de métodos de pontos interiores primais-duais. De fato, se $\sigma$ for uma constante, independente de $n$, então, os métodos de trajetória central são conhecidos como métodos de passo longo. Por outro lado, se $\sigma$ depende de $n$, como por exemplo, $\sigma=\frac{1}{\sqrt{n}}$, então os métodos são conhecidos como métodos de passo curto (PENG; ROOS; TERLAKY, 2000).

É conhecido da literatura (PENG; TERLAKY, 2001; ROOS, June, 2005), que os métodos de passo curto têm complexidade computacional $O\left(\sqrt{n} \log \left(\frac{n}{\epsilon}\right)\right)$, enquanto que os de passo longo convergem em $O\left(n \log \left(\frac{n}{\epsilon}\right)\right)$. A razão para a pior complexidade dos métodos de passo longo, segundo (PENG; ROOS; TERLAKY, 2000) é que em ambos os métodos a mesma função de proximidade $\delta(\mu)$ é utilizada para controlar as iterações. Mas, contrariamente aos resultados teóricos, como afirmam ainda (PENG; ROOS; TERLAKY, 2000), os métodos de passo longo são mais eficientes, na prática, do que os métodos de passo curto.

\subsubsection{Método de Passo Curto}

Este método de passo curto (MPC) inicia com um ponto $\left(x^{0}, y^{0}, z^{0}\right) \in N_{2}(\xi)$ e utiliza valores constantes $\alpha_{k}=1$ e $\sigma_{k}=\sigma(n)$. Cada termo da seqüência $\left(x^{k}, y^{k}, z^{k}\right)$ permanece dentro da vizinhança $N_{2}(\xi)$ e a medida de dualidade $\mu_{k}$ converge linearmente para zero a uma taxa constante $1-\sigma$ (WRIGHT, 1997).

\section{Algoritmo MPC}

Dados: $\xi \in(0,1), \sigma(n) \in[0,1]$ e $\left(x^{0}, y^{0}, z^{0}\right) \in N_{2}(\xi)$.

Para $k=0,1,2, \ldots$

$\sigma_{k}=\sigma$,

Resolva (3.37) para obter $\left(x^{k}, y^{k}, z^{k}\right)$;

Faça $\left(x^{k+1}, y^{k+1}, z^{k+1}\right)=\left(x^{k}, y^{k}, z^{k}\right)+\left(\Delta x^{k}, \Delta y^{k}, \Delta z^{k}\right)$; 
A Figura 3.1 extraída de (WRIGHT, 1997) ilustra claramente o comportamento do algoritmo. Nela, os eixos horizontal e vertical representam, respectivamente, os pontos $x_{1} z_{1}$ e $x_{2} z_{2}$, tal que o caminho central é definido pela reta $x_{2} z_{2}=x_{1} z_{1}$. Neste espaço $(x z)$ não linear as direções de busca são curvas, ao invés de retas.

A solução é a origem e a finalidade é atingir este ponto enquanto mantém as condições de factibilidade em todas as iterações.

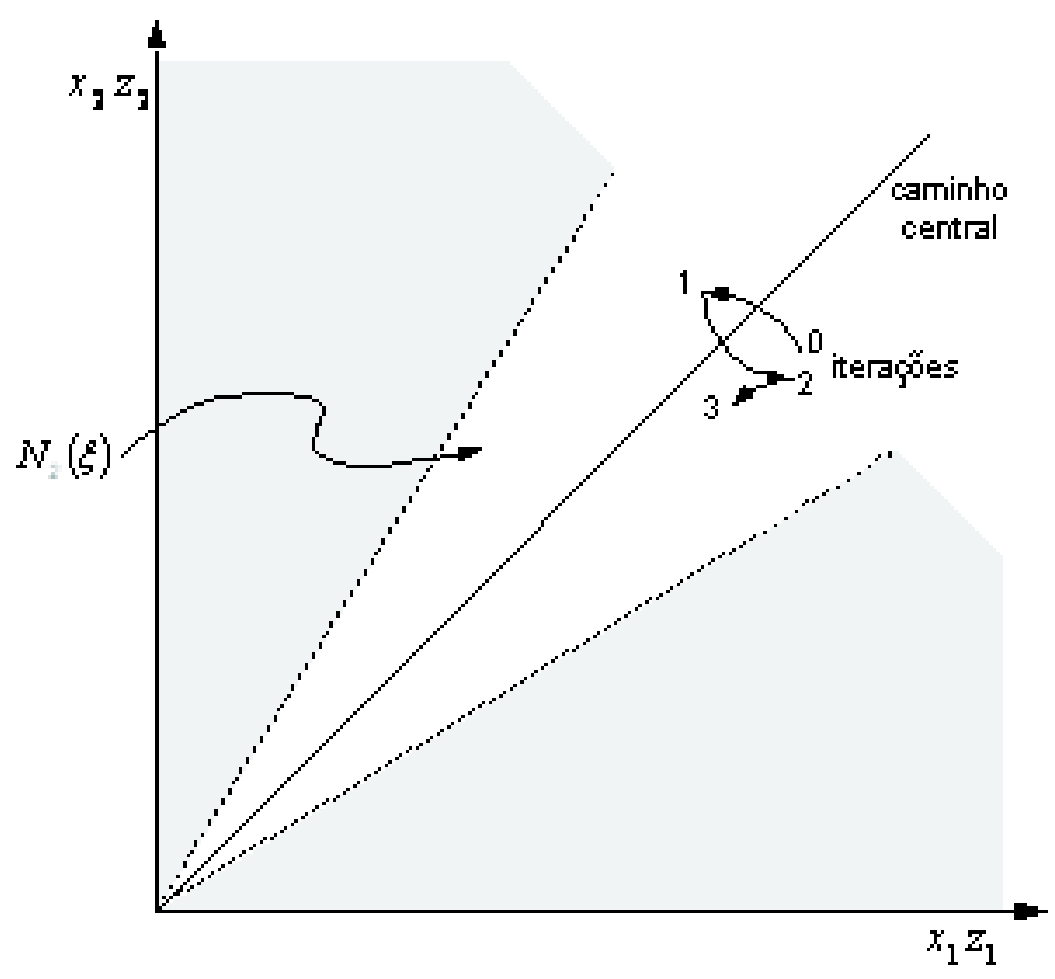

Figura 3.1: Iterações do Algoritmo MPC

Uma dificuldade na análise do algoritmo está em mostrar que cada termo da seqüência $\left(x^{k}, y^{k}, z^{k}\right)$ permanece na vizinhança $N_{2}(\xi)$. Os resultados que seguem, na forma de lemas e teorema, demonstrados por (WRIGHT, 1997), mostram como esta exigência pode ser satisfeita.

Lema 3.1 Se $(x, y, z) \in N_{2}(\xi)$, então,

$$
\|\Delta X \Delta Z e\| \leq \frac{\xi^{2}+n(1-\sigma)^{2}}{2 \sqrt{2}(1-\xi)} \mu .
$$

Este lema fornece um limite para os produtos $X Z e$. 


\subsubsection{Um Teorema de Complexidade Geral para Métodos de Trajetória Central}

A teoria da complexidade é a teoria de algoritmos computacionais. Ela tem desempenhado um papel importante na história da programação linear e métodos de pontos interiores.

A teoria da complexidade faz distinção fundamental entre duas espécies de algoritmos: algoritmos polinomiais, que garantem resolver um dado problema em um tempo que é polinomial com relação a alguma medida do problema; algoritmos exponenciais, que requerem uma quantidade de tempo que é exponencial com relação ao tamanho do problema. Por exemplo, em programação linear, o método simplex é um algoritmo exponencial no pior caso, embora requeira menos tempo computacional na prática do que o esperado pela teoria. O método dos elipsóides de Khachiyan é polinomial, mas surpreendentemente mais lento que o método simplex na prática. O método de Karmarkar e muitos outros métodos de pontos interiores são polinomiais e com desempenho prático muito bom (WRIGHT, 1997).

O teorema a seguir cuja demonstração encontra-se em (WRIGHT, 1997) mostra que se a redução em $\mu$ em cada iteração depende da dimensão $n$ e, se a medida de dualidade inicial $\mu_{0}$ não é muito grande, então o algoritmo tem complexidade polinomial.

Teorema 3.3 Seja $\epsilon \in(0,1)$ dado. Suponha que o algoritmo para resolver (3.25) a (3.28) gere uma seqüência que satisfaz

$$
\mu_{k+1} \leq\left(1-\frac{\xi}{n^{w}}\right) \mu_{k}, \quad k=0,1,2, \ldots
$$

para constantes positivas $\xi$ e w. Suponha, ainda, que o ponto inicial $\left(x^{0}, y^{0}, z^{0}\right)$ satisfaça

$$
\mu_{0} \leq \frac{1}{\epsilon^{\kappa}}
$$

para alguma constante positiva $\kappa$. Então, existe um índice $K$ com

$$
K=O\left(n^{w}|\log \epsilon|\right)
$$

tal que

$$
\mu_{k} \leq \epsilon \quad \forall k \geq K
$$


Grande parte do trabalho na análise de algoritmos está em mostrar que a condição crucial (3.40) é satisfeita.

Lema 3.2 Se $(x, y, z) \in N_{2}(\xi)$, então, temos:

$$
\begin{aligned}
\|X(\alpha) Z(\alpha) e-\mu(\alpha) e\| & \leq|1-\alpha|\|X Z e-\mu e\|+\alpha^{2}\|\Delta X \Delta Z e\| \\
& \leq|1-\alpha| \xi \mu+\alpha^{2}\left[\frac{\xi^{2}+n(1-\sigma)^{2}}{2 \sqrt{2}(1-\xi)}\right] \mu .
\end{aligned}
$$

Este lema, que é um corolário do Lema 3.1, responde à seguinte questão: Até que ponto o termo $(x(\alpha), y(\alpha), z(\alpha))$ distancia do caminho central na norma 2? A notação $(x(\alpha), y(\alpha), z(\alpha))$ significa:

$$
(x(\alpha), y(\alpha), z(\alpha))=\left(x^{k}, y^{k}, z^{k}\right)+\alpha\left(\Delta x^{k}, \Delta y^{k}, \Delta z^{k}\right) .
$$

Teorema 3.4 Sejam $\xi \in(0,1)$ e $\sigma \in(0,1)$ escolhidos de tal forma que satisfaçam

$$
\frac{\xi^{2}+n(1-\sigma)^{2}}{2 \sqrt{2}(1-\xi)} \leq \sigma \xi .
$$

Então, se $(x, y, z) \in N_{2}(\xi)$, temos que $(x(\alpha), y(\alpha), z(\alpha)) \in N_{2}(\xi)$ para todo $\alpha \in$ $[0,1]$

Este teorema define uma relação entre $\xi$ e $\sigma$ e mostra que mesmo um passo completo $(\alpha=1)$ na direção de busca, não fará com que $\left(x^{k+1}, y^{k+1}, z^{k+1}\right)$ saia fora da vizinhança $N_{2}(\xi)$. Assim, no algoritmo MPC, os parâmetros $\xi$ e $\sigma$ devem ser escolhidos de tal forma que satisfaçam a condição (3.43) do Teorema 3.4. A rigor, basta escolher adequadamente o parâmetro $\xi$ e definir $\sigma=1-\frac{\xi}{n^{w}}$ (ver Teorema 3.3) com $w$ uma contante positiva.

\subsection{Método de Passo Longo}

Este método de passo longo(MPL) permite que se combine flexibilidade na escolha do tamanho do passo $\alpha_{k}$ com a utilização de uma vizinhança mais ampla, a saber $N_{-\infty}(\xi)$. Ele gera uma seqüência na vizinhança $N_{-\infty}(\xi)$, que para pequenos valores de $\xi$ (da ordem de $10^{-3}$, por exemplo), praticamente todo o conjunto de pontos estritamente factíveis $S^{0}$ 
pode ser percorrido. Para cada termo da seqüência, o parâmetro de centro $\sigma_{k}$ pode ser escolhido entre dois limites fixos $\sigma_{\min }$ e $\sigma_{\max }$, em $0<\sigma_{\min }<\sigma_{\max }<1$. A direção de pesquisa é obtida pela resolução do sistema

$$
\left[\begin{array}{rrr}
0 & A^{\prime} & I \\
A & 0 & 0 \\
Z & 0 & X
\end{array}\right]\left[\begin{array}{r}
\Delta x^{k} \\
\Delta y^{k} \\
\Delta z^{k}
\end{array}\right]=\left[\begin{array}{r}
0 \\
0 \\
-X Z e+\sigma_{k} \mu_{k} e
\end{array}\right]
$$

e o passo $\alpha_{k}$ é tomado tão grande quanto possível, desde que permaneça dentro da vizinhança $N_{-\infty}(\xi)$.

\section{Algoritmo MPL}

Dados $\xi \in(0,1), \sigma_{\min }$ e $\sigma_{\max }$ tal que $0<\sigma_{\min }<\sigma_{\max }<1$ e $\left(x^{0}, y^{0}, z^{0}\right) \in N_{-\infty}(\xi)$;

Para $k=0,1,2, \ldots$

Escolha $\sigma_{k} \in\left[\sigma_{\min }, \sigma_{\max }\right]$;

Resolva o sistema acima para obter $\left(\Delta x^{k}, \Delta y^{k}, \Delta z^{k}\right)$;

Escolha $\alpha_{k}$ como o maior valor de $\alpha \in[0,1]$ tal que $\left(x^{k}(\alpha), y^{k}(\alpha), z^{k}(\alpha)\right) \in N_{-\infty}(\xi)$;

Faça $\left(x^{k+1}, y^{k+1}, z^{k+1}\right)=\left(x^{k}, y^{k}, z^{k}\right)+\alpha_{k}\left(\Delta x^{k}, \Delta y^{k}, \Delta z^{k}\right)$;

FIM.

A Figura 3.2, extraida de (WRIGHT, 1997) ilustra o comportamento deste método. Nela, vemos que a direção de pesquisa inicia no interior da vizinhança $N_{-\infty}(\xi)$, o que é garantido pelo limite inferior $\sigma_{m i n}$, e move-se em direção ao contorno da vizinhança, mas sempre no interior dela. Além disso, também está garantido que um certo passo mínimo pode ser tomado antes que o contorno de $N_{-\infty}(\xi)$ seja atingido. O lema e o teorema que seguem determinam um limite inferior para $\alpha_{k}$ e uma estimativa correspondente de como reduzir $\mu$ em cada iteração. O último teorema estabelece um resultado de complexidade polinomial. As demonstrações são encontradas em (WRIGHT, 1997).

Lema $3.3 \mathrm{Se}(x, y, z) \in N_{-\infty}(\xi)$, então

$$
\|\Delta X \Delta Z e\| \leq \frac{\left(1+\frac{1}{\xi}\right) n \mu}{2 \sqrt{2}} .
$$

Teorema 3.5 Dados os parâmetros $\xi, \sigma_{\min }$ e $\sigma_{\max }$ do algoritmo $S C P L$, existe uma constante $\psi$ independente de $n$ tal que 


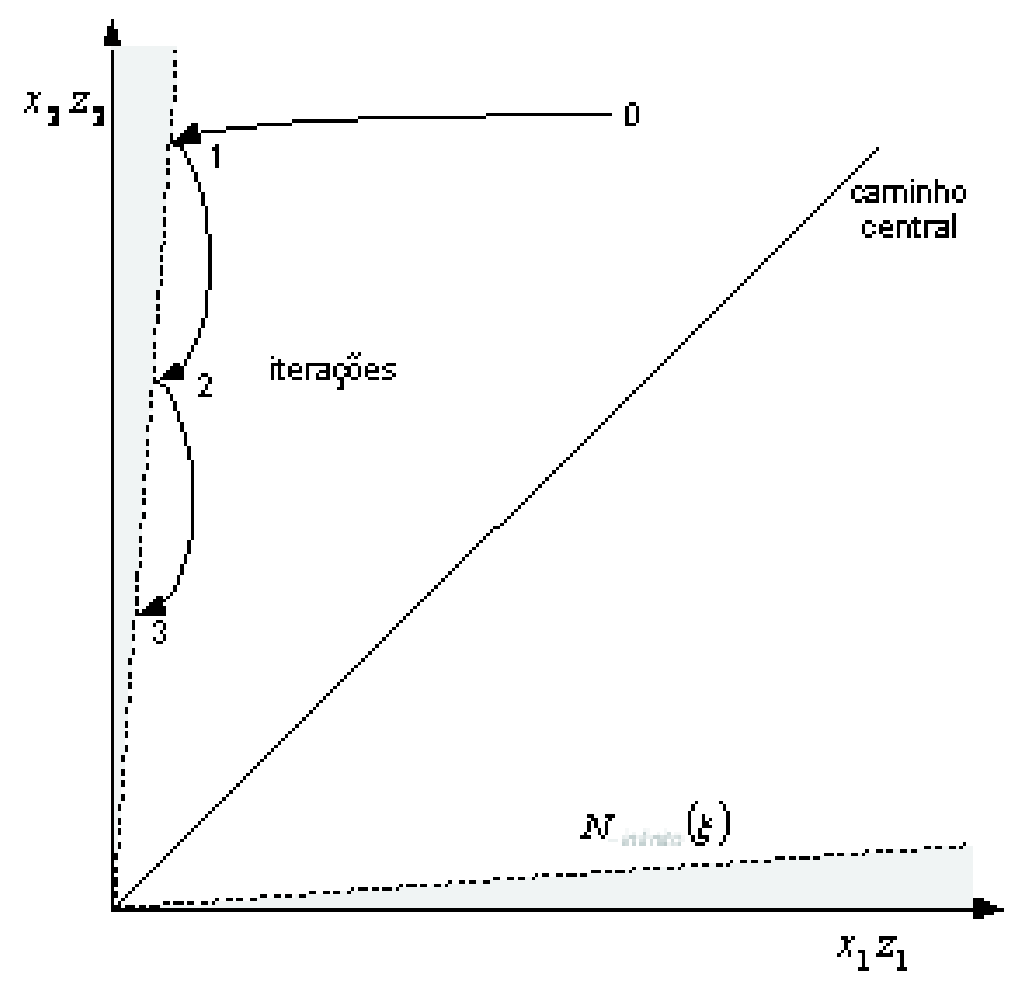

Figura 3.2: Iterações do Algoritmo MPL

$$
\mu_{k+1} \leq\left(1-\frac{\psi}{n}\right) \mu_{k}
$$

para todo $k \geq 0$.

Na demonstração deste teorema, (WRIGHT, 1997) deduz que o termo $\left(x^{k}(\alpha), y^{k}(\alpha), z^{k}(\alpha)\right)$ satisfaz a condição de proximidade para $N_{-\infty}(\xi)$, dada por $x_{i}^{k}(\alpha) z_{i}^{k}(\alpha) \geq \xi \mu_{k}(\alpha)$,se

$$
\alpha \in\left[0, \quad 2 \sqrt{2} \xi \frac{1-\xi \sigma_{k}}{1+\xi n}\right]
$$

Estabelece, ainda, que o parâmetro $\psi$ é definido por:

$$
\psi=2 \sqrt{2} \xi \frac{1-\xi}{1+\xi} \min \left\{\sigma_{\min }\left(1-\sigma_{\min }\right), \sigma_{\max }\left(1-\sigma_{\max }\right)\right\}
$$

Teorema 3.6 Dado $\epsilon>0$ e $\xi \in(0,1)$, suponha que o ponto inicial $\left(x^{0}, y^{0}, z^{0}\right) \in N_{-\infty}(\xi)$ no algoritmo MPL tenha 


$$
\mu_{0} \leq \frac{1}{\epsilon^{\kappa}}
$$

para alguma constante positiva $\kappa$. Então, existe um indice $K \operatorname{com} K=O\left(\operatorname{nlog}\left(\frac{1}{\epsilon}\right)\right)$ tal que $\mu_{k} \leq \epsilon$ para todo $k \geq K$.

\subsubsection{Métodos de Pontos Interiores Infactíveis}

Os algoritmos MPC e MPL exigem que o ponto inicial $\left(x^{0}, y^{0}, z^{0}\right)$ seja estritamente factível em alguma vizinhança do caminho central, isto é, $A x^{0}-b=0$ e $A^{\prime} y^{0}+z^{0}=c$. É possível obter novos métodos a partir da modificação destes de tal forma que não haja exigência de que o ponto inicial $\left(x^{0}, y^{0}, z^{0}\right)$ seja estritamente factível, embora a condição $\left(x^{0}, z^{0}\right)>0$ ainda tenha que ser mantida. Tais métodos são conhecidos como algoritmos de pontos interiores infactíveis(MPII). Neles, as direções de busca são geradas do mesmo modo que são nos algoritmos MPC e MPL e o passo $\alpha_{k}$ é escolhido de forma a garantir que os produtos $x_{i} z_{i}$ não vão prematuramente para zero.

Os passos no método infactível são obtidos considerando o seguinte procedimento: Definimos os resíduos

$$
r_{b}=b-A x, \quad r_{c}=c-A^{\prime} y-z,
$$

e resolvemos as equações de Newton modificadas, para valores do parâmetro de centro $\sigma$,

$$
\left[\begin{array}{rrr}
0 & A^{\prime} & I \\
A & 0 & 0 \\
Z & 0 & X
\end{array}\right]\left[\begin{array}{r}
\Delta x \\
\Delta y \\
\Delta z
\end{array}\right]=\left[\begin{array}{r}
r_{c} \\
r_{b} \\
-X Z e+\sigma \mu e
\end{array}\right] .
$$

Os vetores (3.48) são calculados no ponto $(x, y, z)=\left(x^{k}, y^{k}, z^{k}\right)$, serão denotados por $r_{b}^{k}$ e $r_{c}^{k}$. O método MPII trabalha com a vizinhança $N_{-\infty}(\xi, \beta)$ que é uma extensão de $N_{-\infty}(\xi)$ e inclui pontos infactíveis. Esta vizinhança é definida por (WRIGHT, 1997):

$$
N_{-\infty}(\xi, \beta)=\left\{(x, y, z) /\left\|\left(r_{b}, r_{c}\right)\right\| \leq \frac{\left[\left\|\left(r_{b}^{0}, r_{c}^{0}\right)\right\|\right]}{\mu_{0}} \beta \mu, \quad(x, z)>0, x_{i} z_{i} \geq \xi \mu, \quad i=1,2, \ldots, n\right\}
$$


em que $\xi \in(0,1)$ e $\beta \geq 1$ são parâmetros dados e $\left(r_{b}^{0}, r_{c}^{0}\right)$ e $\mu_{0}$ são avaliados no ponto inicial $\left(x^{0}, y^{0}, z^{0}\right)$. É importante observar que $\beta \geq 1$ para garantir que o ponto inicial $\left(x^{0}, y^{0}, z^{0}\right) \in N_{-\infty}(\xi, \beta)$.

\section{Método MPII}

Dados $\xi, \beta, \sigma_{\min }, \sigma_{\max }, \xi \in(0,1), \beta \geq 1$ e $0<\sigma_{\min }<\sigma_{\max } \geq 0.5$;

Escolha $\left(x^{0}, y^{0}, z^{0}\right) \operatorname{com}\left(x^{0}, z^{0}\right)>0$;

Para $k=0,1,2, \ldots$

Escolha $\sigma_{k} \in\left[\sigma_{\min }, \sigma_{\max }\right]$ e resolva

$$
\left[\begin{array}{rrr}
0 & A^{\prime} & I \\
A & 0 & 0 \\
Z^{k} & 0 & X^{k}
\end{array}\right]\left[\begin{array}{r}
\Delta x^{k} \\
\Delta y^{k} \\
\Delta z^{k}
\end{array}\right]=\left[\begin{array}{r}
r_{c}^{k} \\
r_{b}^{k} \\
-X^{k} Z^{k} e+\sigma_{k} \mu_{k} e
\end{array}\right]
$$

Escolha $\alpha_{\max }$ como sendo o maior valor de $\alpha \in[0,1]$ tal que $\left(x^{k+1}, y^{k+1}, z^{k+1}\right) \in$ $N_{-\infty}(\xi, \beta)$, isto é,

$\left(x^{k}, z^{k}\right)+\alpha\left(\Delta x^{k}, \Delta z^{k}\right) \geq 0$.

Seja $\alpha_{k}=\min \left(1,(1-\xi) \alpha_{\max }\right)$;

Faça $\left(x^{k+1}, y^{k+1}, z^{k+1}\right)=\left(x^{k}, y^{k}, z^{k}\right)+\alpha_{k}\left(\Delta x^{k}, \Delta y^{k}, \Delta z^{k}\right)$

FIM.

Os dois teoremas que seguem, demonstrados em (WRIGHT, 1997), fornecem, respectivamente, a convergência global e a complexidade do algoritmo MPII.

Teorema 3.7 A seqüência $\left\{\mu_{k}\right\}$ gerada pelo algoritmo MPII converge Q-linearmente para zero, e a seqüência formada pelas normas dos resíduos $\left\{\left\|\left(r_{b}^{k}, r_{c}^{k}\right)\right\|\right\}$ converge $R$ linearmente para zero.

Teorema 3.8 Seja $\epsilon>0$ dado. Suponha que estamos iniciando com o ponto $\left(x^{0}, y^{0}, z^{0}\right)=$

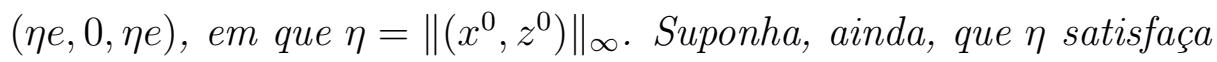

$$
\eta^{2} \leq \frac{C}{\epsilon^{\kappa}}
$$

para algumas constantes positivas $C$ e $\kappa$. Então, existe um índice $K$ com

$$
K=O\left(n^{2}|\log \epsilon|\right)
$$




$$
\mu_{k} \leq \epsilon, \quad \forall \quad k \geq K
$$

\subsubsection{Método Preditor-Corretor}

As implementações dos métodos de pontos interiores foram bastante aprimoradas no período entre 1988 e 1991. Entretanto, em 1992, Mehrotra (MEHROTRA, 1992) publicou um artigo que descreve uma implementação extremamente eficiente que, ainda hoje, é a base para muitas implementações atuais de pontos interiores. Seu método é semelhante ao algoritmo MPII, mas incorpora uma série de modificações e heurísticas que aperfeiçoam o desempenho do código para um grande número de problemas.

O principal custo da implementação do método de Mehrotra é proveniente da solução de dois sistemas:

Um sistema da forma

$$
\left[\begin{array}{rrr}
0 & A^{\prime} & I \\
A & 0 & 0 \\
Z & 0 & X
\end{array}\right]\left[\begin{array}{c}
\Delta x^{k} \\
\Delta y^{k} \\
\Delta z^{k}
\end{array}\right]=\left[\begin{array}{r}
0 \\
0 \\
-X Z e+\sigma_{k} \mu_{k} e
\end{array}\right]
$$

com $\sigma_{k}=0$, para obter o passo afim-escala, e um sistema da forma

$$
\left[\begin{array}{rrr}
0 & A^{\prime} & I \\
A & 0 & 0 \\
Z & 0 & X
\end{array}\right]\left[\begin{array}{c}
\Delta x^{k} \\
\Delta y^{k} \\
\Delta z^{k}
\end{array}\right]=\left[\begin{array}{r}
0 \\
0 \\
-X Z e+\sigma_{k} \mu_{k} e-\Delta X \Delta Z e
\end{array}\right]
$$

para obter a direção de busca.

As matrizes $\Delta X$ e $\Delta Z$ são diagonais a partir das componentes $x$ e $z$ da direção afim-escala já calculada.

Assim, em cada iteração, o método calcula primeiro a direção afim-escala fazendo $\sigma_{k}=0$ (preditor) e depois resolve o segundo sistema definindo $\mu=\frac{x^{\prime} z}{n}$ e $\sigma_{k}=1$ (corretor).

Um fato importante no algoritmo preditor-corretor é que ele utiliza duas vizinhanças, a saber $N_{2}\left(\xi_{1}\right)$ e $N_{2}\left(\xi_{2}\right)$ com $N_{2}\left(\xi_{1}\right) \subset N_{2}\left(\xi_{2}\right)$ (WRIGHT, 1997). O passo preditor reduz o valor de $\mu$ por um fator $(1-\alpha)$, em que $\alpha$ é o passo. O passo corretor deixa $\mu$ inal- 
terado, mas retorna à vizinhança $N_{2}\left(\xi_{1}\right)$, o que permite maior mobilidade para caminhar na próxima iteração.

A Figura 3.3 a seguir, baseada em (WRIGHT, 1997), mostra o procedimento do método de pontos interiores preditor-corretor (MPIPC).

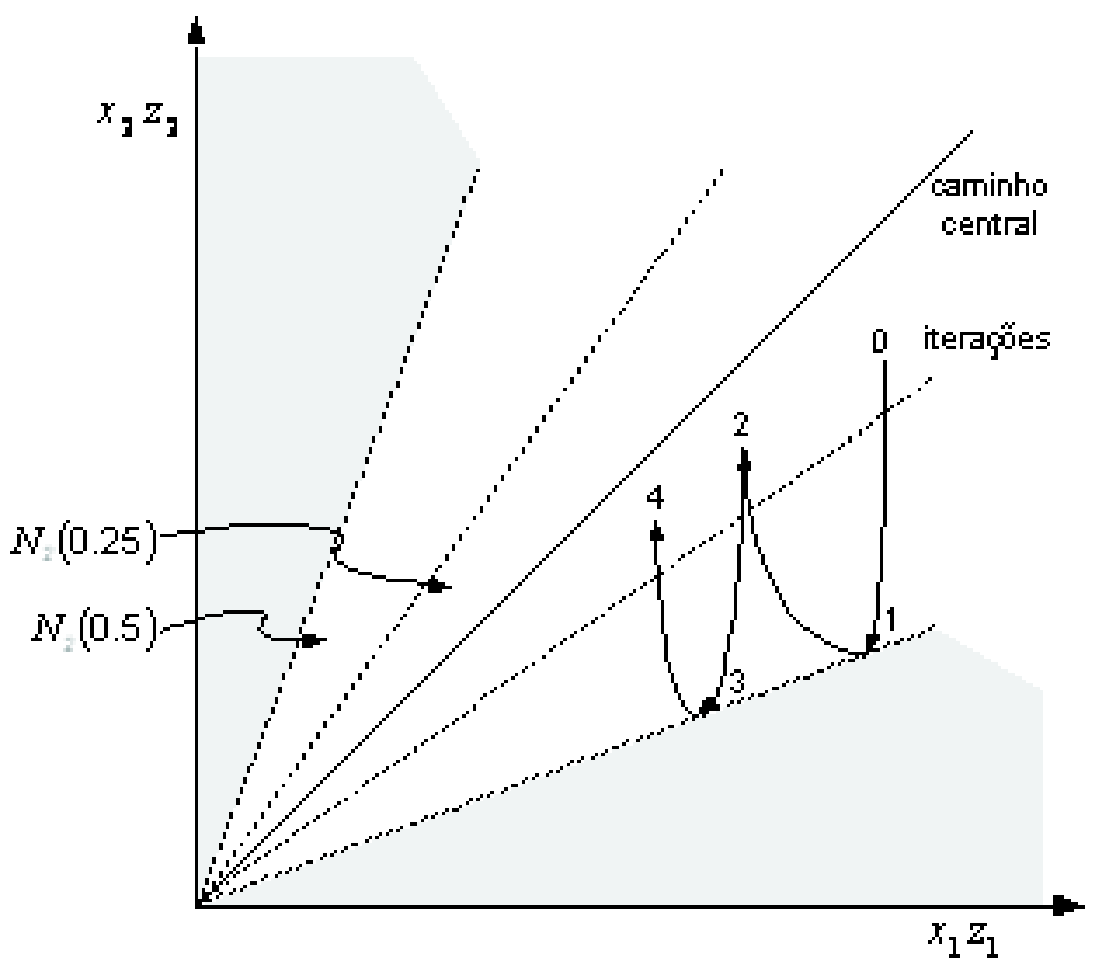

Figura 3.3: Iterações do Algoritmo MPIPC

\section{Método MPIPC}

Dados $\xi_{1}, \xi_{2},\left(x^{0}, y^{0}, z^{0}\right) \in N_{2}\left(\xi_{1}\right)$

Para $k=0,1,2, \ldots$

(Passo Preditor)

Faça $\sigma_{k}=0$ e resolva o sistema anterior para obter $\left(\Delta x^{k}, \Delta y^{k}, \Delta z^{k}\right)$;

Escolha $\alpha_{k} \in[0,1]$ tal que $\left(x^{k+1}, y^{k+1}, z^{k+1},\right) \in N_{2}\left(\xi_{2}\right)$

(Passo Corretor)

Faça $\sigma_{k}=1$ e resolva o sistema anterior para obter $\left(\Delta x^{k}, \Delta y^{k}, \Delta z^{k}\right)$;

Escolha $\alpha_{k} \in[0,1]$ tal que $\left(x^{k+1}, y^{k+1}, z^{k+1}\right)=\left(x^{k}, y^{k}, z^{k}\right)+\alpha_{k}\left(\Delta x^{k}, \Delta y^{k}, \Delta z^{k}\right)$;

FIM. 


\subsection{Conclusão}

Neste capítulo, fornecemos informações gerais sobre otimização, multiplicadores de Lagrange, barreira logarítmica e resultados sobre a existência e unicidade do problema da barreira. Estes conceitos são fundamentais no desenvolvimento e na construção de métodos de pontos interiores.

Organizamos um esboço da teoria dos métodos primais-duais e preditor-corretor, iniciando pelos métodos de trajetória central. Definimos os conceitos de caminho central e vizinhança, apresentamos um algoritmo de passo curto, um algoritmo de passo longo e um método preditor-corretor.

Considerando a natureza deste trabalho, um método de pontos interiores infactível também foi apresentado, bem como alguns resultados de convergência. 


\section{Capítulo 4}

\section{Métodos de Pontos Interiores para Problema de Fluxo de Potência Ótimo DC}

\subsection{Introdução}

A minimização de perdas no pré-despacho de um sistema de potência hidroelétrico é um problema de planejamento operacional de curto prazo. Nesta aplicação, curto prazo significa a operação horária durante um dia ou uma semana. Este problema pode ser modelado por meio da minimização de uma função quadrática com variáveis separáveis, representando as perdas no sistema hidráulico de geração e de transmissão no sistema de potência, sujeito às restrições lineares representando o fluxo de potência ativo. O conjunto de restrições lineares pode ser dividido em blocos que se repetem a cada intervalo de tempo, representando o sistema elétrico em cada estádio (hora). Estes blocos são acoplados por restrições adicionais (OLIVEIRA; NEPOMUCENO; SOARES, 2005). O objetivo deste capítulo é minimizar as perdas na geração e transmissão do sistema de potência, usando o método de pontos interiores (MPI). 


\subsection{O Modelo Dinâmico}

Consideremos, novamente, o modelo (1.7) descrito na Seção (1.2) do Capítulo 1, ou seja,

$$
\begin{array}{ccc}
\operatorname{minimizar} & \frac{\alpha}{2} \sum_{k=1}^{t}\left[\left(f^{k}\right)^{\prime} R f^{k}+r^{\prime} f^{k}\right]+\frac{\beta}{2} \sum_{k=1}^{t}\left[\left(p^{k}\right)^{\prime} Q p^{k}+c^{\prime} p^{k}\right] & \\
\text { sujeito a } & k=1, \cdots, t \\
A f^{k}=E p^{k}-l^{k}, & k=1, \cdots, t \\
T f^{k}=0, & k=1, \cdots, t \\
f^{\min } \leq f^{k} \leq f^{\max }, & k=1, \cdots, t \\
p^{\min } \leq p^{k} \leq p^{\max }, & \\
\sum_{k=1}^{t} p^{k}=q . &
\end{array}
$$

\subsection{Desenvolvimento do Método}

Para simplificar o desenvolvimento do método de ponto interior, faremos as seguintes mudanças de variáveis:

$$
\begin{aligned}
\tilde{f}^{k} & =f^{k}-f^{\text {min }} \\
\tilde{f}^{\text {max }} & =f^{\text {max }}-f^{\text {min }} \\
\tilde{p}^{k} & =p^{k}-p^{\text {min }} \\
\tilde{p}^{\text {max }} & =p^{\text {max }}-p^{\text {min }} .
\end{aligned}
$$

Com esta notação, as equações (4.4) e (4.5) têm seus limites inferiores transformados em zero, de tal forma que o modelo anterior pode ser reescrito como:

$$
\begin{aligned}
& \operatorname{minimizar} \frac{\alpha}{2} \sum_{k=1}^{t}\left[\left(\tilde{f}^{k}\right)^{\prime} R \tilde{f}^{k}+c_{\tilde{f}}^{\prime} \tilde{f}^{k}\right]+\frac{\beta}{2} \sum_{k=1}^{t}\left[\left(\tilde{p}^{k}\right)^{\prime} Q \tilde{p}^{k}+c_{\tilde{p}}^{\prime} \tilde{p}^{k}\right] \\
& \text { sujeito a } \\
& A \tilde{f}^{k}-E \tilde{p}^{k}=p^{m i n}-l^{k}-A f^{m i n}=\tilde{l}^{k}, \\
& k=1, \cdots, t \\
& T \tilde{f}^{k}=-T f^{\min }=\tilde{l}^{T}, \\
& k=1, \cdots, t \\
& 0 \leq \tilde{f}^{k} \leq \tilde{f}^{\text {max }}, \\
& k=1, \cdots, t \\
& 0 \leq \tilde{p}^{k} \leq \tilde{p}^{\max }, \\
& k=1, \cdots, t \\
& \sum_{k=1}^{t} \tilde{p}^{k}=q-\sum_{k=1}^{t} p^{\min }=\tilde{q}
\end{aligned}
$$


em que:

$$
\begin{aligned}
& c_{\tilde{f}}^{\prime}=2\left(f^{\text {min }}\right)^{\prime} R+r^{\prime} \\
& c_{\tilde{p}}^{\prime}=2\left(p^{\text {min }}\right)^{\prime} Q+c^{\prime}
\end{aligned}
$$

e

$$
\begin{aligned}
\left(f^{\text {min }}\right)^{\prime} R f^{\text {min }} & =\text { constante } \\
\left(p^{\text {min }}\right)^{\prime} Q p^{\text {min }}+c^{\prime} p^{\text {min }} & =\text { constante }
\end{aligned}
$$

Estas constantes podem ser desprezadas na solução do modelo, pois não alteram o problema de minimização.

Acrescentando as variáveis de folga $s_{\tilde{f}}^{k}$ e $s_{\tilde{p}}^{k}$ temos, finalmente, o modelo primal na forma padrão:

$$
\begin{array}{crl}
\operatorname{minimizar} & \frac{\alpha}{2} \sum_{k=1}^{t}\left[\left(\tilde{f}^{k}\right)^{\prime} R \tilde{f}^{k}+c_{\tilde{f}}^{\prime} \tilde{f}^{k}\right]+\frac{\beta}{2} \sum_{k=1}^{t}\left[\left(\tilde{p}^{k}\right)^{\prime} Q \tilde{p}^{k}+c_{\tilde{p}}^{\prime} \tilde{p}^{k}\right] & \\
\text { sujeito a } & k=1, \cdots, t \\
A \tilde{f}^{k}-E \tilde{p}^{k}=\tilde{l}^{k}, & k=1, \cdots, t \\
T \tilde{f}^{k}=\tilde{l}^{T}, & k=1, \cdots, t \\
\tilde{f}^{k}+s_{\tilde{f}}^{k}=\tilde{f}^{\max }, & k=1, \cdots, t \\
\tilde{p}^{k}+s_{\tilde{p}}^{k}=\tilde{p}^{\text {max }}, & \\
\sum_{k=1}^{t} \tilde{p}^{k}=\tilde{q} & \\
\left(\tilde{f}^{k}, s_{\tilde{f}}^{k}\right) \geq 0, \quad\left(\tilde{p}^{k}, s_{\tilde{p}}^{k}\right) \geq 0 . &
\end{array}
$$

As restrições (4.8) e (4.9) podem ser combinadas de tal forma que resultem em uma equação. De fato, reescrevendo-as na forma matricial, temos:

$\mathrm{Ou}$

$$
\left[\begin{array}{rr}
A & -E \\
T & 0
\end{array}\right]\left[\begin{array}{l}
\tilde{f}^{k} \\
\tilde{p}^{k}
\end{array}\right]=\left[\begin{array}{l}
\tilde{l}^{k} \\
\tilde{l}^{T}
\end{array}\right]
$$

$$
\left[\begin{array}{ll}
B & -\hat{E}
\end{array}\right]\left[\begin{array}{l}
\tilde{f}^{k} \\
\tilde{p}^{k}
\end{array}\right]=\left[\begin{array}{l}
\hat{l}^{k} \\
\hat{l}^{T}
\end{array}\right]
$$


em que a matriz $B$ é constituída pelas linhas justapostas de $A$ e $T$ e, portanto, de ordem $m+(n-m+1)=n+1$ por $n$. A matriz $\hat{E}$ tem $\operatorname{ordem}(n+1) \times g$, com as últimas $n-m+1$ linhas iguais a zero. Os vetores $\hat{l}^{k}$ e $\hat{l}^{T}$ são definidos por:

$$
\hat{l}^{k}=\left[\begin{array}{c}
\tilde{l}^{k} \\
0
\end{array}\right], \quad \hat{l}^{T}=\left[\begin{array}{c}
\tilde{l}^{T} \\
0
\end{array}\right] .
$$

Com estas notações, o problema de pré-despacho primal pode ser reescrito como:

$$
\begin{gathered}
\text { minimizar } \frac{\alpha}{2} \sum_{k=1}^{t}\left[\left(\tilde{f}^{k}\right)^{\prime} R \tilde{f}^{k}+c_{\tilde{f}}^{\prime} \tilde{f}^{k}\right]+\frac{\beta}{2} \sum_{k=1}^{t}\left[\left(\tilde{p}^{k}\right)^{\prime} Q \tilde{p}^{k}+c_{\tilde{p}}^{\prime} \tilde{p}^{k}\right] \\
\text { sujeito a } \tilde{f}^{k}-\hat{E} \tilde{p}^{k}=\hat{l}^{k} \\
\tilde{f}^{k}+s_{\tilde{f}}^{k}=\tilde{f}^{\max } \\
\tilde{p}^{k}+s_{\tilde{p}}^{k}=\tilde{p}^{\text {max }} \\
\sum_{k=1}^{t} \tilde{p}^{k}=\tilde{q} \\
\left(\tilde{f}^{k}, s_{\tilde{f}}^{k}, \tilde{p}^{k}, s_{\tilde{p}}^{k}\right) \geq 0 .
\end{gathered}
$$

\subsection{Problema de Pré-Despacho Dual}

O objetivo desta seção é escrever o problema de pré-despacho dual associado ao problema primal. Reescrevendo as restrições do problema primal, na forma matricial, temos:

$$
\left[\begin{array}{rrrrr}
D_{1} & 0 & 0 & \cdots & 0 \\
0 & D_{2} & 0 & \cdots & 0 \\
0 & 0 & D_{3} & \cdots & 0 \\
\vdots & \vdots & \ddots & \vdots & \vdots \\
0 & 0 & 0 & \cdots & D_{k} \\
I_{\tilde{p}^{1}} & I_{\tilde{p}^{2}} & I_{\tilde{p}^{3}} & \cdots & I_{\tilde{p}^{k}}
\end{array}\right]\left[\begin{array}{r}
v^{1} \\
v^{2} \\
v^{3} \\
\vdots \\
v^{k}
\end{array}\right]=\left[\begin{array}{r}
d^{1} \\
d^{2} \\
d^{3} \\
\vdots \\
d^{k}
\end{array}\right]
$$

em que, 


$$
\begin{gathered}
D_{k}=\left[\begin{array}{rrrr}
B & -\hat{E} & 0 & 0 \\
I & 0 & I & 0 \\
0 & I & 0 & I
\end{array}\right], \\
I_{\tilde{p}^{k}}=\left[\begin{array}{llll}
0 & I & 0 & 0
\end{array}\right], \\
\left(v^{k}\right)^{\prime}=\left[\begin{array}{llll}
\tilde{f}^{k} & \tilde{p}^{k} & s_{\tilde{f}}^{k} & s_{\tilde{p}}^{k}
\end{array}\right],
\end{gathered}
$$

e

$$
\left(d^{k}\right)^{\prime}=\left[\begin{array}{llll}
\hat{l}^{k} & \tilde{f}^{\max } & \tilde{p}^{\max } & \tilde{q} \cdot
\end{array}\right]
$$

Sejam:

$$
G=\left[\begin{array}{rrrrr}
D_{1} & 0 & 0 & \cdots & 0 \\
0 & D_{2} & 0 & \cdots & 0 \\
0 & 0 & D_{3} & \cdots & 0 \\
\vdots & \vdots & \ddots & \vdots & \vdots \\
0 & 0 & 0 & \cdots & D_{k} \\
I_{\tilde{p}^{1}} & I_{\tilde{p}^{2}} & I_{\tilde{p}^{3}} & \cdots & I_{\tilde{p}^{k}}
\end{array}\right]
$$

e

$$
b^{\prime}=\left[\begin{array}{llll}
d^{1} & d^{2} & \cdots & d^{k}
\end{array}\right] .
$$

Com esta notação, o problema dual é dado por (BAZARAA; JARVIS; SHERALI, 1990):

$$
\begin{gathered}
\operatorname{maximizar} \quad\left\{b^{\prime} y-\sum_{k=1}^{t}\left[\frac{\alpha}{2}\left(\tilde{f}^{k}\right)^{\prime} R \tilde{f}^{k}+\frac{\beta}{2}\left(\tilde{p}^{k}\right)^{\prime} Q \tilde{p}^{k}\right]\right\} \\
\text { sujeito a } \quad B^{\prime} y_{1}^{k}+y_{2}^{k} \leq c_{\tilde{f}}+R \tilde{f}^{k} \\
-\hat{E}^{\prime} y_{1}^{k}+y_{3}^{k}+y_{a} \leq c_{\tilde{p}}+Q \tilde{p}^{k} \\
y_{2}^{k} \leq 0 \\
y_{3}^{k} \leq 0 .
\end{gathered}
$$


- $y_{1}^{k}$ é de ordem $(n+1) \times 1, \quad \mathrm{k}=1,2, \ldots, \mathrm{t}$

- $y_{2}^{k}$ é de ordem $n \times 1, \quad \mathrm{k}=1,2, \ldots, \mathrm{t}$

- $y_{3}^{k}$ e $y_{a}$ são de ordem $g \times 1, \quad \mathrm{k}=1,2, \ldots, \mathrm{t}$.

Em termos matriciais o problema dual assume a forma:

$$
\begin{gathered}
\operatorname{maximizar} \quad\left\{b^{\prime} y-\sum_{k=1}^{t}\left[\frac{\alpha}{2}\left(\tilde{f}^{k}\right)^{\prime} R \tilde{f}^{k}+\frac{\beta}{2}\left(\tilde{p}^{k}\right)^{\prime} Q \tilde{p}^{k}\right]\right\} \\
\text { sujeito a } \\
G^{\prime} y \leq c \\
y \quad \text { irrestrito }
\end{gathered}
$$

em que

$$
y^{\prime}=\left[\begin{array}{llll}
y_{1}^{k} & y_{2}^{k} & y_{3}^{k} & y_{a}
\end{array}\right]
$$

e

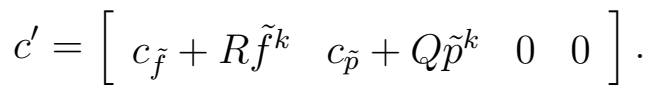

Substituindo as variáveis $y_{1}^{k}, y_{2}^{k}$ e $y_{3}^{k}$, respectivamente, por $y_{\tilde{f}}^{k},-w_{\tilde{f}}^{k}$ e $-w_{\tilde{p}}^{k}$, o problema pode ser reescrito na seguinte forma:

$$
\begin{gathered}
\operatorname{maximizar} \sum_{k=1}^{t}\left\{\left(\hat{l}^{k}\right)^{\prime} y_{\tilde{f}}^{k}-\left(\tilde{f}^{\max }\right)^{\prime} w_{\tilde{f}}^{k}-\left(\tilde{p}^{\max }\right)^{\prime} w_{\tilde{p}}^{k}+(\tilde{q})^{\prime} y_{a}\right. \\
\left.-\frac{\alpha}{2}\left[\left(\tilde{f}^{k}\right)^{\prime} R \tilde{f}^{k}\right]-\frac{\beta}{2}\left[\left(\tilde{p}^{k}\right)^{\prime} Q \tilde{p}^{k}\right]\right\} \\
\text { sujeito a } \quad B^{\prime} y_{\tilde{f}}^{k}-w_{\tilde{f}}^{k}-R \tilde{f}^{k} \leq c_{\tilde{f}} \\
-\hat{E}^{\prime} y_{\tilde{f}}^{k}-w_{\tilde{p}}^{k}+y_{a}-Q \tilde{p}^{1} \leq c_{\tilde{p}} \\
-w_{\tilde{f}}^{k} \leq 0 \\
-w_{\tilde{p}}^{k} \leq 0 .
\end{gathered}
$$


Acrescentando as variáveis de folga $\left(z_{\tilde{f}}^{k}, z_{\tilde{p}}^{k}\right)$, temos, finalmente, o problema dual de fluxo em redes:

$$
\begin{gathered}
\operatorname{maximizar} \sum_{k=1}^{t}\left\{\left(\hat{l}^{k}\right)^{\prime} y_{\tilde{f}}^{k}-\left(\tilde{f}^{\max }\right)^{\prime} w_{\tilde{f}}^{k}-\left(\tilde{p}^{\max }\right)^{\prime} w_{\tilde{p}}^{k}+(\tilde{q})^{\prime} y_{a}\right. \\
\left.-\frac{\alpha}{2}\left[\left(\tilde{f}^{k}\right)^{\prime} R \tilde{f}^{k}\right]-\frac{\beta}{2}\left[\left(\tilde{p}^{k}\right)^{\prime} Q \tilde{p}^{k}\right]\right\} \\
\text { sujeito a } \quad B^{\prime} y_{\tilde{f}}^{k}-w_{\tilde{f}}^{k}-R \tilde{f}^{k}+z_{\tilde{f}}^{k}=c_{\tilde{f}} \\
-\hat{E}^{\prime} y_{\tilde{f}}^{k}-w_{\tilde{p}}^{k}+y_{a}-Q \tilde{p}^{k}+z_{\tilde{p}}^{k}=c_{\tilde{p}} \\
\left(z_{\tilde{f}}^{k}, w_{\tilde{f}}^{k}, z_{\tilde{p}}^{k}, w_{\tilde{p}}^{k}\right) \geq 0, \quad y_{\tilde{f}}^{k}, \quad y_{a} \quad \text { livres. }
\end{gathered}
$$

\subsection{Condições de Otimalidade}

As condições de otimalidade para os problemas primal e dual são dadas pela factibilidade primal e dual e pelas condições de complementaridade.

1. Factibilidade Primal

$$
\begin{gathered}
B \tilde{f}^{k}-\hat{E} \tilde{p}^{k}=\hat{l}^{k} \\
\tilde{f}^{k}+s_{\tilde{f}}^{k}=\tilde{f}^{\max } \\
\tilde{p}^{k}+s_{\tilde{p}}^{k}=\tilde{p}^{\max } \\
\sum_{k=1}^{t} \tilde{p}^{k}=\tilde{q} \\
\left(\tilde{f}^{k}, s_{\tilde{f}}^{k}, \tilde{p}^{k}, s_{\tilde{p}}^{k}\right) \geq 0 .
\end{gathered}
$$

2. Factibilidade Dual

$$
\begin{gathered}
B^{\prime} y_{\tilde{f}}^{k}-w_{\tilde{f}}^{k}-R \tilde{f}^{k}+z_{\tilde{f}}^{k}=c_{\tilde{f}} \\
-\hat{E}^{\prime} y_{\tilde{f}}^{k}-w_{\tilde{p}}^{k}+y_{a}-Q \tilde{p}^{k}+z_{\tilde{p}}^{k}=c_{\tilde{p}} \\
\left(z_{\tilde{f}}^{k}, w_{\tilde{f}}^{k}, z_{\tilde{p}}^{k}, w_{\tilde{p}}^{k}\right) \geq 0, \quad y_{\tilde{f}}^{k}, \quad y_{a} \quad \text { livres } .
\end{gathered}
$$


3. Condições de Complementaridade

$$
\begin{aligned}
\tilde{F}^{k} Z_{\tilde{f}}^{k} e & =0 \\
S_{\tilde{f}}^{k} W_{\tilde{f}}^{k} e & =0 \\
\tilde{P}^{k} Z_{\tilde{p}}^{k} e & =0 \\
S_{\tilde{p}}^{k} W_{\tilde{p}}^{k} e & =0 .
\end{aligned}
$$

\subsection{Método de Newton}

Aplicando o método de Newton (Apêndice A) às condições de otimalidade (4.20) a (4.29), obtemos o seguinte sistema linear:

$$
J d=r
$$

em que $J$ representa a matriz Jacobiana dada por:

$$
\left[\begin{array}{rrrrr}
M_{1} & 0 & 0 & \cdots & 0 \\
0 & M_{2} & 0 & \cdots & 0 \\
0 & 0 & M_{3} & \cdots & 0 \\
\vdots & \vdots & \ddots & \vdots & \vdots \\
0 & 0 & 0 & \cdots & M_{k} \\
I_{\tilde{p}^{1}} & I_{\tilde{p}^{2}} & I_{\tilde{p}^{3}} & \cdots & I_{\tilde{p}^{k}}
\end{array}\right]
$$

em que cada $M_{k}$ corresponde à submatriz

$$
\left[\begin{array}{rrrrrrrrrr}
B & -\hat{E} & 0 & 0 & 0 & 0 & 0 & 0 & 0 & 0 \\
I & 0 & I & 0 & 0 & 0 & 0 & 0 & 0 & 0 \\
0 & I & 0 & I & 0 & 0 & 0 & 0 & 0 & 0 \\
-R & 0 & 0 & 0 & B^{\prime} & -I & I & 0 & 0 & 0 \\
0 & -Q & 0 & 0 & -\hat{E}^{\prime} & 0 & 0 & -I & I & I \\
Z_{\tilde{f}}^{1} & 0 & 0 & 0 & 0 & 0 & \tilde{F}^{1} & 0 & 0 & 0 \\
0 & 0 & W_{\tilde{f}}^{1} & 0 & 0 & S_{\tilde{f}}^{1} & 0 & 0 & 0 & 0 \\
0 & Z_{\tilde{p}}^{1} & 0 & 0 & 0 & 0 & 0 & 0 & \tilde{P}^{1} & 0 \\
0 & 0 & 0 & W_{\tilde{p}}^{1} & 0 & 0 & 0 & S_{\tilde{p}}^{1} & 0 & 0
\end{array}\right],
$$

cada $I_{\tilde{p}^{k}}$ corresponde ao vetor 


$$
I_{\tilde{p}^{k}}=\left[\begin{array}{llllllllll}
0 & I & 0 & 0 & 0 & 0 & 0 & 0 & 0 & 0
\end{array}\right],
$$

$d$ representa as direções de Newton associadas às variáveis do problema:

$$
d^{\prime}=\left[\begin{array}{llllllllll}
d \tilde{f}^{k} & d \tilde{p}^{k} & d s_{\tilde{f}}^{k} & d s_{\tilde{p}}^{k} & d y_{\tilde{f}}^{k} & d w_{\tilde{f}}^{k} & d z_{\tilde{f}}^{k} & d w_{\tilde{p}}^{k} & d z_{\tilde{p}}^{k} & d y_{a}
\end{array}\right]
$$

e $r$ representa o vetor dos resíduos, cujas componentes são dadas por:

$$
\begin{aligned}
r_{l} & =\hat{l}^{k}-B \tilde{f}^{k}+\hat{E} \tilde{p}^{k} \\
r_{f} & =\tilde{f}^{m a x}-\tilde{f}^{k}-s_{\tilde{f}}^{k} \\
r_{p} & =\tilde{p}^{m a x}-\tilde{p}^{k}-s_{\tilde{p}}^{k} \\
r_{y} & =c_{\tilde{f}}-B^{\prime} y_{\tilde{f}}^{k}+w_{\tilde{f}}^{k}+R \tilde{f}^{k}-z_{\tilde{f}}^{k} \\
r_{g} & =c_{\tilde{p}}+\hat{E}^{\prime} y_{\tilde{f}}^{k}+w_{\tilde{p}}^{k}-y_{a}+Q \tilde{p}^{k}-z_{\tilde{p}}^{k} \\
r_{z f} & =\mu e-\tilde{F}^{k} Z_{\tilde{f}}^{k} e \\
r_{w f} & =\mu e-S_{\tilde{f}}^{k} W_{\tilde{f}}^{k} e \\
r_{z p} & =\mu e-\tilde{P}^{k} Z_{\tilde{p}}^{k} e \\
r_{w p} & =\mu e-S_{\tilde{p}}^{k} W_{\tilde{p}}^{k} e \\
r_{m} & =\tilde{q}-\sum_{k=1}^{t} \tilde{p}^{k} .
\end{aligned}
$$

Portanto, as direções de Newton são dadas pelo seguinte sistema linear: 


$$
\begin{aligned}
B d \tilde{f}^{k}-\hat{E} d \tilde{p}^{k} & =r_{l} \\
d \tilde{f}^{k}+d s_{\tilde{f}}^{k} & =r_{f} \\
d \tilde{p}^{k}+d s_{\tilde{p}}^{k} & =r_{p} \\
B^{\prime} d y_{\tilde{f}}^{k}-d w_{\tilde{f}}^{k}-R d \tilde{f}^{k}+d z_{\tilde{f}}^{k} & =r_{y} \\
-\hat{E}^{\prime} d y_{\tilde{f}}^{k}-d w_{\tilde{p}}^{k}+d y_{a}-Q d \tilde{p}^{k}+d z_{\tilde{p}}^{k} & =r_{g} \\
\tilde{F}^{k} d z_{\tilde{f}}^{k}+Z_{\tilde{f}}^{k} d \tilde{f}^{k} & =r_{z f} \\
S_{\tilde{f}}^{k} d w_{\tilde{f}}^{k}+W_{\tilde{f}}^{k} d s_{\tilde{f}}^{k} & =r_{w f} \\
\tilde{P}^{k} d z_{\tilde{p}}^{k}+Z_{\tilde{p}}^{k} d \tilde{p}^{k} & =r_{z p} \\
S_{\tilde{p}}^{k} d w_{\tilde{p}}^{k}+W_{\tilde{p}}^{k} d s_{\tilde{p}}^{k} & =r_{w p} \\
\sum_{k=1}^{t} d \tilde{p}^{k} & =r_{m} .
\end{aligned}
$$

Nosso próximo passo consiste em resolver o sistema constituído pelas Equações (4.30) a (4.39). Para isto, vamos utilizar o conceito de substituição de variáveis com o objetivo de reduzir a dimensão do sistema linear.

\subsection{Redução do Sistema Linear}

Eliminando as variáveis $d z_{\tilde{f}}^{k}, d w_{\tilde{f}}^{k}, d z_{\tilde{p}}^{k}, d w_{\tilde{p}}^{k}$, presentes, respectivamente, nas Equações (4.35), (4.36), (4.37) e (4.38), temos:

$$
\begin{aligned}
d z_{\tilde{f}}^{k} & =\left(\tilde{F}^{k}\right)^{-1}\left[r_{z f}-Z_{\tilde{f}}^{k} d \tilde{f}^{k}\right] \\
d w_{\tilde{f}}^{k} & =\left(S_{\tilde{f}}^{k}\right)^{-1}\left[r_{w f}-W_{\tilde{f}}^{k} d s_{\tilde{f}}^{k}\right] \\
d z_{\tilde{p}}^{k} & =\left(\tilde{P}^{k}\right)^{-1}\left[r_{z p}-Z_{\tilde{p}}^{k} d \tilde{p}^{k}\right] \\
d w_{\tilde{p}}^{k} & =\left(S_{\tilde{p}}^{k}\right)^{-1}\left[r_{w p}-W_{\tilde{p}}^{k} d s_{\tilde{p}}^{k}\right] .
\end{aligned}
$$

Substituindo os valores das variáveis explicitadas em (4.40) a (4.43), nas variáveis correspondentes em (4.33) e (4.34), temos:

$$
\begin{aligned}
B^{\prime} d y_{\tilde{f}}^{k}-\left[R+\left(\tilde{F}^{k}\right)^{-1} Z_{\tilde{f}}^{k}\right] d \tilde{f}^{k}+\left(S_{\tilde{f}}^{k}\right)^{-1} W_{\tilde{f}}^{k} d s_{\tilde{f}}^{k} & =r_{y}+\left(S_{\tilde{f}}^{k}\right)^{-1} r_{w f}-\left(\tilde{F}^{k}\right)^{-1} r_{z f} \\
-\hat{E}^{\prime} d y_{\tilde{f}}^{k}-\left[Q+\left(\tilde{P}^{k}\right)^{-1} Z_{\tilde{p}}^{k}\right] d \tilde{p}^{k}+\left(S_{\tilde{p}}^{k}\right)^{-1} W_{\tilde{p}}^{k} d s_{\tilde{p}}^{k}+d y_{a} & =r_{g}+\left(S_{\tilde{p}}^{k}\right)^{-1} r_{w p}-\left(\tilde{P}^{k}\right)^{-1} r_{z p} .
\end{aligned}
$$


Eliminando as variáveis $d s_{\tilde{f}}^{k}$ e $d s_{\tilde{p}}^{k}$, respectivamente, nas equações (4.31) e (4.32) e substituindo nas equações (4.44) e (4.45), obtemos:

$$
\begin{aligned}
B^{\prime} d y_{\tilde{f}}^{k}-\left[R+\left(\tilde{F}^{k}\right)^{-1} Z_{\tilde{f}}^{k}+\left(S_{\tilde{f}}^{k}\right)^{-1} W_{\tilde{f}}^{k}\right] d \tilde{f}^{k} & =r_{y}+\left(S_{\tilde{f}}^{k}\right)^{-1} r_{w f}-\left(\tilde{F}^{k}\right)^{-1} r_{z f}-\left(S_{\tilde{f}}^{k}\right)^{-1} W_{\tilde{f}}^{k} r_{f} \\
-\hat{E}^{\prime} d y_{\tilde{f}}^{k}-\left[Q+\left(\tilde{P}^{k}\right)^{-1} Z_{\tilde{p}}^{k}+\left(S_{\tilde{p}}^{k}\right)^{-1} W_{\tilde{p}}^{k}\right] d \tilde{p}^{k}+d y_{a} & =r_{g}+\left(S_{\tilde{p}}^{k}\right)^{-1} r_{w p}-\left(\tilde{P}^{k}\right)^{-1} r_{z p}-\left(S_{\tilde{p}}^{k}\right)^{-1} W_{\tilde{p}}^{k} r_{p} .
\end{aligned}
$$

Com estas substituições, o conjunto de Equações (4.30) a (4.38) fica reduzido à:

$$
\begin{aligned}
B d \tilde{f}^{k}-\hat{E} d \tilde{p}^{k} & =r_{l} \\
B^{\prime} d y_{\tilde{f}}^{k}-\left[R+\left(\tilde{F}^{k}\right)^{-1} Z_{\tilde{f}}^{k}+\left(S_{\tilde{f}}^{k}\right)^{-1} W_{\tilde{f}}^{k}\right] d \tilde{f}^{k} & =r_{y}+\left(S_{\tilde{f}}^{k}\right)^{-1} r_{w f}-\left(\tilde{F}^{k}\right)^{-1} r_{z f}-\left(S_{\tilde{f}}^{k}\right)^{-1} W_{\tilde{f}}^{k} r_{f} \\
\text { ou } & \\
y_{\tilde{f}}^{k}-\left[Q+\left(\tilde{P}^{k}\right)^{-1} Z_{\tilde{p}}^{k}+\left(S_{\tilde{p}}^{k}\right)^{-1} W_{\tilde{p}}^{k}\right] d \tilde{p}^{k}+d y_{a} & =r_{g}+\left(S_{\tilde{p}}^{k}\right)^{-1} r_{w p}-\left(\tilde{P}^{k}\right)^{-1} r_{z p}-\left(S_{\tilde{p}}^{k}\right)^{-1} W_{\tilde{p}}^{k} r_{p} \\
B d \tilde{f}^{k}-\hat{E} d \tilde{p}^{k} & =r_{l} \\
B^{\prime} d y_{\tilde{f}}^{k}-D_{\tilde{f}}^{k} d \tilde{f}^{k} & =r_{a} \\
-\hat{E}^{\prime} d y_{\tilde{f}}^{k}-D_{\tilde{p}}^{k} d \tilde{p}^{k}+d y_{a} & =r_{b}
\end{aligned}
$$$$
-\hat{E}^{\prime} d y_{\tilde{f}}^{k}-\left[Q+\left(\tilde{P}^{k}\right)^{-1} Z_{\tilde{p}}^{k}+\left(S_{\tilde{p}}^{k}\right)^{-1} W_{\tilde{p}}^{k}\right] d \tilde{p}^{k}+d y_{a}=r_{g}+\left(S_{\tilde{p}}^{k}\right)^{-1} r_{w p}-\left(\tilde{P}^{k}\right)^{-1} r_{z p}-\left(S_{\tilde{p}}^{k}\right)^{-1} W_{\tilde{p}}^{k} r_{p},
$$

em que

$$
\begin{aligned}
D_{\tilde{f}}^{k} & =R+\left(\tilde{F}^{k}\right)^{-1} Z_{\tilde{f}}^{k}+\left(S_{\tilde{f}}^{k}\right)^{-1} W_{\tilde{f}}^{k} \\
D_{\tilde{p}}^{k} & =Q+\left(\tilde{P}^{k}\right)^{-1} Z_{\tilde{p}}^{k}+\left(S_{\tilde{p}}^{k}\right)^{-1} W_{\tilde{p}}^{k} \\
r_{a} & =r_{y}+\left(S_{\tilde{f}}^{k}\right)^{-1} r_{w f}-\left(\tilde{F}^{k}\right)^{-1} r_{z f}-\left(S_{\tilde{f}}^{k}\right)^{-1} W_{\tilde{f}}^{k} r_{f} \\
r_{b} & =r_{g}+\left(S_{\tilde{p}}^{k}\right)^{-1} r_{w p}-\left(\tilde{P}^{k}\right)^{-1} r_{z p}-\left(S_{\tilde{p}}^{k}\right)^{-1} W_{\tilde{p}}^{k} r_{p} .
\end{aligned}
$$

Eliminando $d \tilde{f}^{k}$ e $d \tilde{p}^{k}$, respectivamente, nas Equações (4.47) e (4.48), obtemos:

$$
\begin{aligned}
d \tilde{f}^{k} & =\left(D_{\tilde{f}}^{k}\right)^{-1}\left[-r_{a}+B^{\prime} d y_{\tilde{f}}^{k}\right] \\
d \tilde{p}^{k} & =\left(D_{\tilde{p}}^{k}\right)^{-1}\left[-r_{b}-E^{\prime} d y_{\tilde{f}}^{k}+d y_{a}\right] .
\end{aligned}
$$

Substituindo em (4.46), temos: 


$$
\left[B\left(D_{\tilde{f}}^{k}\right)^{-1} B^{\prime}+\hat{E}\left(D_{\tilde{p}}^{k}\right)^{-1} \hat{E}^{\prime}\right] d y_{\tilde{f}}^{k}-\hat{E}\left(D_{\tilde{p}}^{k}\right)^{-1} d y_{a}=r_{l}+B\left(D_{\tilde{f}}^{k}\right)^{-1} r_{a}-\hat{E}\left(D_{\tilde{p}}^{k}\right)^{-1} r_{b}
$$

O sistema linear composto pelas equações (4.30) a (4.39) fica reduzido, então, às seguintes equações:

$$
\begin{aligned}
{\left[B\left(D_{\tilde{f}}^{k}\right)^{-1} B^{\prime}+\hat{E}\left(D_{\tilde{p}}^{k}\right)^{-1} \hat{E}^{\prime}\right] d y_{\tilde{f}}^{k}-\hat{E}\left(D_{\tilde{p}}^{k}\right)^{-1} d y_{a} } & =r \\
\sum_{k=1}^{t} d \tilde{p}^{k} & =r_{m}
\end{aligned}
$$

em que

$$
r=r_{l}+B\left(D_{\tilde{f}}^{k}\right)^{-1} r_{a}-\hat{E}\left(D_{\tilde{p}}^{k}\right)^{-1} r_{b}
$$

Substituindo a equação (4.50) na equação de acoplamento (4.52), temos:

$$
\sum_{k=1}^{t}\left(D_{\tilde{p}}^{k}\right)^{-1}\left[d y_{a}-\hat{E}^{\prime} d y_{\tilde{f}}^{k}\right]=r_{m}+\sum_{k=1}^{2}\left(D_{\tilde{p}}^{k}\right)^{-1} r_{b}
$$

O próximo passo é resolver o sistema formado pelas equações (4.51) e (4.54).

\subsection{Estudo da Estrutura Matricial}

Consideremos as equações (4.51) e (4.54) escritas na seguinte forma:

$$
\begin{aligned}
{\left[B\left(D_{\tilde{f}}^{k}\right)^{-1} B^{\prime}+D^{k}\right] d y_{\tilde{f}}^{k}-\hat{E}\left(D_{\tilde{p}}^{k}\right)^{-1} d y_{a} } & =r \\
\sum_{k=1}^{t}\left(D_{\tilde{p}}^{k}\right)^{-1}\left[d y_{a}-\hat{E}^{\prime} d y_{\tilde{f}}^{k}\right] & =r_{m}+\sum_{k=1}^{t}\left(D_{\tilde{p}}^{k}\right)^{-1} r_{b}
\end{aligned}
$$

em que

$D^{k}=\hat{E}\left(D_{\tilde{p}}^{k}\right)^{-1} \hat{E}^{\prime}$ tem ordem $(n+1) \times(n+1)$ e é tal que suas $m$ primeiras linhas e $m$ primeiras colunas constitui uma matriz diagonal que é exatamente a matriz $\left(D_{\tilde{p}}^{k}\right)^{-1}$, enquanto que o restante de suas entradas é zero (OLIVEIRA; NEPOMUCENO; SOARES, 2005). Isto significa que esta matriz é singular. Eliminando $d y_{\tilde{f}}^{k}$ em (4.55) e substituindo em (4.56), obtemos o sistema: 
$\sum_{k=1}^{t}\left\{\left(D_{\tilde{p}}^{k}\right)^{-1}-\left(D_{\tilde{p}}^{k}\right)^{-1} \hat{E}^{\prime} M^{-1} \hat{E}\left(D_{\tilde{p}}^{k}\right)^{-1}\right\} d y_{a}=r_{m}+\sum_{k=1}^{t}\left(D_{\tilde{p}}^{k}\right)^{-1}\left[r_{b}+\hat{E}^{\prime} M^{-1} r\right]$

em que

$$
M=B\left(D_{\tilde{f}}^{k}\right)^{-1} B^{\prime}+D^{k} .
$$

A resolução direta do sistema (4.57) exige muito esforço computacional, pois, a matriz $M$ tem dimensão do número de linhas, enquanto que $d y_{a}$ tem dimensão do número de geradores. Para contornar este problema, procederemos da seguinte forma:

1. Formamos a matriz quadrada $\tilde{B}$ a partir de $B$ acrescentando a esta uma coluna correspondente a um vetor canônico $e^{\prime}=\left[\begin{array}{lllll}0 & 0 & \ldots & 0 & 1\end{array}\right]$, isto é, $\tilde{B}=\left[\begin{array}{ll}B & e\end{array}\right]$. Este fato é crucial, pois, a matriz $\tilde{B}$ resultante é quadrada e não singular. Além disso, devemos acrescentar à matriz $\left(D_{\tilde{f}}^{k}\right)^{-1}$ uma linha e uma coluna para ajustar a dimensão do produto envolvido. Para isto, retiramos da matriz $D^{k}$ a j-ésima linha e a j-ésima coluna, $j=1, \ldots, m$, cuja intersecção seja diferente de zero. Isto equivale a eliminar uma barra de geração da matriz $D^{k}$. Esta, por sua vez, permanece com a mesma dimensão, mas com zeros no lugar correspondente à j-ésima linha e j-ésima coluna retiradas. Com estas modificações, vamos chamar a matriz $\left(D_{\tilde{f}}^{k}\right)^{-1}$ de $\tilde{D}_{\tilde{f}}^{k}$ e a matriz $D^{k}$ de $\tilde{D}^{k}$. Conseqüentemente, a matriz $M$ e o vetor $r$ assumem a forma:

$$
\tilde{M}=\tilde{B} \tilde{D}_{\tilde{f}}^{k} \tilde{B}^{\prime}+\tilde{D}^{k}
$$

com dimensão $(n+1) \times(n+1)$

$$
\tilde{r}=r_{l}+\tilde{B} \tilde{D}_{\tilde{f}}^{k} r_{a}-\hat{E}\left(\tilde{D}_{\tilde{p}}^{k}\right)^{-1} r_{b}
$$

Assim, a Equação (4.57) pode ser reescrita como:

$$
\sum_{k=1}^{t}\left\{\left(D_{\tilde{p}}^{k}\right)^{-1}-\left(D_{\tilde{p}}^{k}\right)^{-1} \hat{E}^{\prime} \tilde{M}^{-1} \hat{E}\left(D_{\tilde{p}}^{k}\right)^{-1}\right\} d y_{a}=r_{m}+\sum_{k=1}^{t}\left(D_{\tilde{p}}^{k}\right)^{-1}\left[r_{b}+\hat{E}^{\prime} \tilde{M}^{-1} r\right]
$$




$$
\left[\tilde{B} \tilde{D}_{\tilde{f}}^{k} \tilde{B}^{\prime}+\tilde{D}^{k}\right] d \hat{y}_{\tilde{f}}^{k}=\tilde{r}
$$

podemos resolver (4.59) em duas etapas. Na primeira, um sistema linear contendo apenas a matriz $\tilde{B} \tilde{D}_{\tilde{f}}^{k} \tilde{B}^{\prime}$ será resolvido. Neste caso, haverá a necessidade de calcular as inversas de $\tilde{B}$ e $\tilde{B}^{\prime}$. Como estas matrizes não variam com o tempo, apenas uma decomposição das mesmas será necessária e pode ser feita antes da aplicação do método iterativo. Na segunda etapa a fórmula de Sherman-Morrison-Woodbury (DUFF; ERISMAN; REID, 1986) será aplicada para a obtenção de $d \hat{y}_{\tilde{f}}^{k}$.

- Primeira Etapa

Definindo,

$$
\left[\tilde{B} \tilde{D}_{\tilde{f}}^{k} \tilde{B}^{\prime}\right] d \hat{\hat{y}}_{\tilde{f}}^{k}=\tilde{r}, \quad k=1,2, \ldots, t
$$

podemos calcular $d \hat{\hat{y}}_{\tilde{f}}^{k}$ como segue:

$$
d \hat{\hat{y}}_{\tilde{f}}^{k}=\left[\tilde{B} \tilde{D}_{\tilde{f}}^{k} \tilde{B}^{\prime}\right]^{-1} \tilde{r}, \quad k=1,2, \ldots, t
$$

ou

$$
d \hat{\hat{y}}_{\tilde{f}}^{k}=\left[\left(\tilde{B}^{\prime}\right)^{-1}\left(\tilde{D}_{\tilde{f}}^{k}\right)^{-1} \tilde{B}^{-1}\right] \tilde{r}, \quad k=1,2, \ldots, t
$$

- Segunda Etapa

A fórmula de Sherman-Morrison-Woodbury expressa em termos de inversa de matriz tem a forma (DUFF; ERISMAN; REID, 1986):

$$
\left(C+U S V^{\prime}\right)^{-1}=C^{-1}-C^{-1} U\left(S^{-1}+V^{\prime} C^{-1} U\right)^{-1} V^{\prime} C^{-1}
$$

em que, nesta fórmula, $U$ e $V$ são matrizes de ordem $p \times q$ e $S$ é uma matriz de ordem $q \times q$. A fim de utilizar a fórmula (4.62) para resolver o sistema (4.59), sejam: 


$$
\begin{aligned}
C & =\tilde{B} \tilde{D}_{\tilde{f}}^{k} \tilde{B}^{\prime} \\
U S V^{\prime} & =\tilde{D}^{k}
\end{aligned}
$$

em que:

- $U$ é tal que suas colunas são as colunas da matriz identidade e de dimensão $(n+1) \times(g)$. Portanto, $U=\hat{E}$.

- $S$ é diagonal, de dimensão $g \times g$, cujos elementos da diagonal são exatamente os elementos não nulos da diagonal de $\tilde{D}^{k}$.

- $V^{\prime}=U^{\prime}$

Por exemplo, seja

$$
\tilde{D}^{k}=\left[\begin{array}{rrrrrr}
\frac{1}{d_{1}} & 0 & 0 & 0 & 0 & 0 \\
0 & 0 & 0 & 0 & 0 & 0 \\
0 & 0 & \frac{1}{d_{3}} & 0 & 0 & 0 \\
0 & 0 & 0 & 0 & 0 & 0 \\
0 & 0 & 0 & 0 & 0 & 0 \\
0 & 0 & 0 & 0 & 0 & 0
\end{array}\right]
$$

em que a segunda linha e segunda coluna foram retiradas para compor $\tilde{D}_{\tilde{f}}^{k}$. Observando que $m=3$ e $n=5$, então as matrizes $U$ e $S$ são definidas por:

$$
\begin{gathered}
U=\left[\begin{array}{ll}
1 & 0 \\
0 & 0 \\
0 & 1 \\
0 & 0 \\
0 & 0 \\
0 & 0
\end{array}\right] \\
S=\left[\begin{array}{cc}
\frac{1}{d_{1}} & 0 \\
0 & \frac{1}{d_{3}}
\end{array}\right]
\end{gathered}
$$


Nosso objetivo é resolver o sistema (4.59),isto é,

$$
d \hat{y}_{\tilde{f}}^{k}=\left[\tilde{B} \tilde{D}_{\tilde{f}}^{k} \tilde{B}^{\prime}+\tilde{D}^{k}\right]^{-1} \tilde{r}, \quad k=1,2, \ldots, t .
$$

Para isto vamos calcular a inversa da matriz $\tilde{M}$ que aparece no lado direito da equação (4.65). Utilizando a fórmula (4.62) e a notação dada nas equações (4.63) e (4.64), temos:

$$
\left[\tilde{B} \tilde{D}_{\tilde{f}}^{k} \tilde{B}^{\prime}+\tilde{D}^{k}\right]^{-1}=\left(\tilde{B} \tilde{D}_{\tilde{f}}^{k} \tilde{B}^{\prime}\right)^{-1}-\left(\tilde{B} \tilde{D}_{\tilde{f}}^{k} \tilde{B}^{\prime}\right)^{-1} \hat{E} Z^{-1} \hat{E}^{\prime}\left(\tilde{B} \tilde{D}_{\tilde{f}}^{k} \tilde{B}^{\prime}\right)^{-1}
$$

em que

$$
Z=S^{-1}+\hat{E}^{\prime}\left(\tilde{B} \tilde{D}_{\tilde{f}}^{k} \tilde{B}^{\prime}\right)^{-1} \hat{E}
$$

é uma matriz simétrica, positiva definida, tem dimensão do número de geradores e pode ser decomposta pelo método de Cholesky (GOLUB; LOAN, 1996). Assim, o esforço computacional para calcular sua inversa em cada tempo não é significativo. Multiplicando ambos os membros da Equação (4.66) por $\tilde{r}$ e utilizando as Equações (4.61) e (4.65), obtemos:

$$
d \hat{y}_{\tilde{f}}^{k}=d \hat{\hat{y}}_{\tilde{f}}^{k}-\left(\tilde{B}^{\prime}\right)^{-1}\left(\tilde{D}_{\tilde{f}}^{k}\right)^{-1} \tilde{B}^{-1} \hat{E} Z^{-1} \hat{E}^{\prime} d \hat{\hat{y}}_{\tilde{f}}^{k}
$$

Definindo $W=\tilde{B}^{-1} \hat{E}$, podemos reescrever a equação anterior na forma,

$$
d \hat{y}_{\tilde{f}}^{k}=d \hat{\hat{y}}_{\tilde{f}}^{k}-\left(\tilde{B}^{\prime}\right)^{-1}\left(\tilde{D}_{\tilde{f}}^{k}\right)^{-1} W Z^{-1} \hat{E}^{\prime} d \hat{\hat{y}}_{\tilde{f}}^{k}
$$

3. Para Calcular $d y_{a}$, substituímos (4.66) em (4.58) e utilizamos a Equação (4.67). Reorganizando os termos, obtemos:

$$
\sum_{k=1}^{t}\left\{\left(D_{\tilde{p}}^{k}\right)^{-1}-\left(D_{\tilde{p}}^{k}\right)^{-1} N\left(D_{\tilde{p}}^{k}\right)^{-1}\right\} d y_{a}=r_{m}+\sum_{k=1}^{t}\left(D_{\tilde{p}}^{k}\right)^{-1}\left[r_{b}+\hat{E}^{\prime} d \hat{y}_{\tilde{f}}^{k}\right]
$$

em que 


$$
N=W^{\prime}\left(D_{\tilde{p}}^{k}\right)^{-1} W-W^{\prime}\left(D_{\tilde{p}}^{k}\right)^{-1} W Z^{-1} W^{\prime}\left(D_{\tilde{p}}^{k}\right)^{-1} W
$$

\subsection{Métodos Primal-Dual e Preditor-Corretor}

Definindo os vetores $x=\left(\tilde{f}^{k}, s_{\tilde{f}}^{k}, \tilde{p}^{k}, s_{\tilde{p}}^{k}\right), y=\left(y_{\tilde{f}}^{k}\right)$ e $z=\left(z_{\tilde{f}}^{k}, w_{\tilde{f}}^{k}, z_{\tilde{p}}^{k}, w_{\tilde{p}}^{k}\right)$, a aplicação do método de Newton às condições de otimalidade resulta nos métodos Primal-Dual via Fluxo de Potência Ótimo DC (PDFPO) e Preditor-Corretor via Fluxo de Potência Ótimo DC (PCFPO):

\section{Método PDFPO}

Dados $\xi, \quad \xi \in(0,1)$ e $\sigma=\frac{1}{\sqrt{n}}, \quad n=2 t(n+g)$;

Escolha $\left(x^{0}, y^{0}, z^{0}\right) \in N_{-\infty}(\xi), \operatorname{com}\left(x^{0}, z^{0}\right)>0, \quad y^{0}=0 \quad$ e $\quad y_{a}^{0}=0$.

Para $k=0,1,2, \ldots$

Escolha $\sigma^{k}=\sigma$;

Faça $\mu^{k}=\sigma^{k}\left(\frac{\gamma^{k}}{n^{2} \sqrt{n}}\right)$, se $\gamma^{k} \geq 1$ e $\mu^{k}=\sigma^{k}\left(\frac{\left(\gamma^{k}\right)^{2}}{n}\right)$, se $\gamma<1, \gamma^{k}=\left(x^{k}\right)^{\prime} z^{k}$.

Calcule as direções de Newton $\Delta x^{k}, \Delta y^{k}, \Delta z^{k}$ e $\Delta y_{a}^{k}$.

Calcule $\alpha_{p}^{k}=\frac{-\tau}{\min _{i}\left(\frac{\Delta x_{i}^{k}}{x_{i}^{k}}\right)}$ e $\alpha_{d}^{k}=\frac{-\tau}{\min _{i}\left(\frac{\Delta z_{i}^{k}}{z_{i}^{k}}\right)}, \tau \in(0,1)$.

Faça $\alpha^{k}=\min \left(1, \alpha_{p}^{k}, \alpha_{d}^{k}\right)$,tal que $\left(x^{k}, y^{k}, z^{k}, y_{a}^{k}\right) \in N_{-\infty}(\xi)$;

Faça $\left(x^{k+1}, y^{k+1}, z^{k+1}, y_{a}^{k+1}\right)=\left(x^{k}, y^{k}, z^{k}, y_{a}^{k}\right)+\alpha^{k}\left(\Delta x^{k}, \Delta y^{k}, \Delta z^{k}, \Delta y_{a}^{k}\right)$

FIM.

\section{Método PCFPO}

Dados $\xi \in(0,1), \quad n=2 t(n+g)$;

Escolha $\left(x^{0}, y^{0}, z^{0}\right) \in N_{-\infty}(\xi), \operatorname{com}\left(x^{0}, z^{0}\right)>0, \quad y^{0}=0 \quad$ e $\quad y_{a}^{0}=0$.

Para $k=0,1,2, \ldots$

(Passo Preditor)

Faça $\sigma^{k}=0$; e calcule as direções de Newton $\Delta x^{k}, \Delta y^{k}, \Delta z^{k}$ e $\Delta y_{a}^{k}$.

Calcule $\alpha_{p}^{k}=\frac{-\tau}{\min _{i}\left(\frac{\Delta x_{i}^{k}}{x_{i}^{k}}\right)}$ e $\alpha_{d}^{k}=\frac{-\tau}{\min _{i}\left(\frac{\Delta z_{i}^{k}}{z_{i}^{k}}\right)}, \tau \in(0,1)$.

Faça $\alpha^{k}=\min \left(1, \alpha_{p}^{k}, \alpha_{d}^{k}\right)$, talque $\left(x^{k}, y^{k}, z^{k}, y_{a}^{k}\right) \in N_{-\infty}(\xi)$;

(Passo Corretor)

Faça $\sigma^{k}=1$; e calcule as direções de Newton $\Delta x^{k}, \Delta y^{k}, \Delta z^{k}$ e $\Delta y_{a}^{k}$. 
Calcule $\alpha_{p}^{k}=\frac{-\tau}{\min _{i}\left(\frac{\Delta x_{i}^{k}}{x_{i}^{k}}\right)}$ e $\alpha_{d}^{k}=\frac{-\tau}{\min _{i}\left(\frac{\Delta z_{i}^{k}}{z_{i}^{k}}\right)}, \tau \in(0,1)$

Faça $\alpha^{k}=\min \left(1, \alpha_{p}^{k}, \alpha_{d}^{k}\right)$;

Faça $\left(x^{k+1}, y^{k+1}, z^{k+1}, y_{a}^{k+1}\right)=\left(x^{k}, y^{k}, z^{k}, y_{a}^{k}\right)+\alpha^{k}\left(\Delta x^{k}, \Delta y^{k}, \Delta z^{k}, \Delta y_{a}^{k}\right)$

FIM.

\subsection{Conclusão}

Neste capítulo, reescrevemos o modelo dinâmico para o problema de minimização de perdas no pré-despacho de um sistema de potência hidroelétrico, introduzimos simplificações por meio de mudanças de variáveis e acrescentamos variáveis de folga para obter o modelo primal na forma padrão. Escrevemos o problema dual associado, bem como as condições de otimalidade, visando aplicar o método de Newton para a solução do problema em questão, pelo método primal-dual e preditor corretor. Fizemos a redução do sistema linear resultante da aplicação do método primal-dual e preditor corretor e, a fim de otimizar a implementação computacional, estudamos a estrutura matricial resultante, objetivando reduzir o tamanho do sistema para a ordem do número de geradores $(g \times g)$. Apresentamos, ainda, os métodos PDFPO e PCFPO que serviram de base para a implementação, respectivamente, dos métodos de pontos interiores para os problemas primal-dual e preditor-corretor, associados ao problema de fluxo de potência ótimo DC. 


\title{
Capítulo 5
}

\section{Métodos de Pontos Interiores para}

\author{
Problema de Fluxo de Potência
}

\section{Ótimo DC usando o Princípio do}

\section{Mínimo Esforço}

\subsection{Introdução}

O despacho ótimo de potência ativa utilizando o modelo DC foi formulado, como visto no Capítulo 4, como um modelo de fluxos em redes com restrições adicionais. Uma vantagem desta abordagem é que, com representação independente das leis de Kirchhoff, os fluxos de potência são representados explicitamente permitindo acesso direto tanto às restrições de capacidade como às perdas no sistema de transmissão (ALMEIDA, 87; OLIVEIRA; NEPOMUCENO; SOARES, 2005). Neste capítulo, os Métodos de Pontos Interiores Primal-Dual foram desenvolvidos para o problema de minimização das perdas na geração e transmissão do pré-despacho DC de um sistema de potência hidroelétrico com base no princípio do mínimo esforço. Além disso, a estrutura matricial resultante da aplicação do Método de Pontos Interiores Primal-Dual foi explorada no sentido de se obter uma implementação computacional eficiente. Finalmente, foi proposto uma nova heurística com base em um vetor de perturbação, cujo método primal-dual resultante mostrou-se eficiente na prática, principalmente porque produziu uma redução significativa no número 


\subsection{O Modelo de Fluxo em Redes}

Consideremos, novamente, a formulação matemática do modelo de otimização do problema de perdas no pré-despacho de um sistema de potência hidrelétrico, como visto no Capítulo 4.

$$
\begin{array}{ccc}
\text { minimizar } \frac{\alpha}{2} \sum_{k=1}^{t}\left[\left(f^{k}\right)^{\prime} R f^{k}+r^{\prime} f^{k}\right]+\frac{\beta}{2} \sum_{k=1}^{t}\left[\left(p^{k}\right)^{\prime} Q p^{k}+c^{\prime} p^{k}\right] & \\
\text { sujeito a } & k=1, \cdots, t \\
A f^{k}=E p^{k}-l^{k}, & k=1, \cdots, t \\
T f^{k}=0, & k=1, \cdots, t \\
f^{\text {min }} \leq f^{k} \leq f^{\max }, & k=1, \cdots, t \\
p^{\text {min }} \leq p^{k} \leq p^{\max }, & \\
\sum_{k=1}^{t} p^{k}=q . &
\end{array}
$$

Nosso próximo passo é aplicar o princípio do mínimo esforço a este modelo.

\subsection{Princípio do Mínimo Esforço}

O princípio do mínimo esforço consiste em simplificar o modelo de fluxo em redes por meio da aproximação

$$
f^{k}=X^{-1} A^{\prime} \theta^{k}=U \theta^{k}, \quad U=X^{-1} A^{\prime}, \quad k=1,2, \ldots, t,
$$

em que $X$ corresponde à matriz diagonal das reatâncias das linhas $\left(X \in R^{n \times n}\right)$ e $\theta$ o vetor dos ângulos das tensões (ALMEIDA, 87).

Com a notação dada pela Equação (5.7), algumas expressões do modelo de fluxo em redes podem ser alteradas, isto é:

- Na Equação (5.1), para $k=1, \ldots, t$,

$$
\left(f^{k}\right)^{\prime} R f^{k}+r^{\prime} f^{k}=\left(U \theta^{k}\right)^{\prime} R\left(U \theta^{k}\right)+r^{\prime} U \theta^{k}=\left(\theta^{k}\right)^{\prime} U^{\prime} R U \theta^{k}+r^{\prime} U \theta^{k}=\left(\theta^{k}\right)^{\prime} \tilde{B} \theta^{k}+c_{\theta}^{\prime} \theta^{k}
$$


em que $\tilde{B}=U^{\prime} R U$ e $c_{\theta}^{\prime}=r^{\prime} U$.

- Na Equação (5.2):

$$
A f^{k}=A U \theta^{k}=B \theta^{k}, \quad k=1,2, \ldots, t
$$

em que $B=A U$.

- Na Equação (5.3):

$$
T f^{k}=T U \theta^{k}=\tilde{T} \theta^{k}, \quad k=1,2, \ldots, t
$$

em que $\tilde{T}=T U$.

- Na Equação (5.4):

$$
f^{\min } \leq f^{k} \leq f^{\max } \Rightarrow f^{\min } \leq U \theta^{k} \leq f^{\max } .
$$

Em virtude da introdução do Princípio do Mínimo Esforço (PME) no modelo de fluxo em redes e o fato de supormos que não existem linhas de transmissão nos limites, a Equação (5.3) passa a ser redundante e pode ser desprezada (OLIVEIRA; SOARES, 2000). Portanto, o modelo de fluxo em redes com a inclusão do PME assume a forma simplificada:

$$
\begin{array}{rlrl}
\operatorname{minimizar} & \frac{\alpha}{2} \sum_{k=1}^{t}\left[\left(\theta^{k}\right)^{\prime} \tilde{B} \theta^{k}+c_{\theta}^{\prime} \theta^{k}\right]+\frac{\beta}{2} \sum_{k=1}^{t}\left[\left(p^{k}\right)^{\prime} Q p^{k}+c^{\prime} p^{k}\right] & \\
\text { sujeito a } & B \theta^{k}-E p^{k}=-l^{k}, & k=1, \cdots, t \\
f^{\text {min }} \leq U \theta^{k} \leq f^{\max }, & k=1, \cdots, t \\
p^{\text {min }} \leq p^{k} \leq p^{\max }, & & \\
\sum_{k=1}^{t} p^{k} & =q . &
\end{array}
$$

Na restrição de desigualdade (5.11) devemos transformar o limite inferior em zero, obtendo: 


$$
0 \leq \tilde{p}^{k} \leq \tilde{p}^{\max }, \quad k=1,2, \cdots, t
$$

em que, $\tilde{p}^{k}=p^{k}-p^{\min }$ e $\tilde{p}^{\max }=p^{\max }-p^{\min }$.

Observando que $\theta^{k}$ é uma variável livre, a restrição (5.10) corresponde às seguintes duas desigualdades:

$$
\begin{gathered}
U \theta^{k} \leq f^{\max }, \quad k=1,2, \cdots, t \\
U \theta^{k} \geq f^{\text {min }}, \quad k=1,2, \cdots, t .
\end{gathered}
$$

Introduzindo estas modificações ao problema (5.8) a (5.12), podemos reescrevê-lo como:

$$
\begin{array}{crl}
\operatorname{minimizar} & \frac{\alpha}{2} \sum_{k=1}^{t}\left[\left(\theta^{k}\right)^{\prime} \tilde{B} \theta^{k}+c_{\theta}^{\prime} \theta^{k}\right]+\frac{\beta}{2} \sum_{k=1}^{t}\left[\left(\tilde{p}^{k}\right)^{\prime} Q \tilde{p}^{k}+c_{p}^{\prime} \tilde{p}^{k}+\text { const }\right] & \\
\text { sujeito a } & & k=1, \cdots(5.16) \\
B \theta^{k}-E \tilde{p}^{k}=E p^{\text {min }} p^{\text {min }}-l^{k}=\tilde{l}^{k}, & k=1, \cdots(5 . t 18) \\
U \theta^{k} \leq f^{\max }, & k=1, \cdots(5 . t 19) \\
U \theta^{k} \geq f^{\text {min }}, & k=1, \cdots(5 . t 20) \\
\tilde{p}^{k} \leq \tilde{p}^{\text {max }}, & \\
\sum_{k=1}^{t} \tilde{p}^{k}=q-\sum_{k=1}^{t} p^{\text {min }}=\tilde{q} & (5.21)
\end{array}
$$

em que,

$$
\begin{array}{r}
c_{p}^{\prime}=2\left(p^{m i n}\right)^{\prime} Q+c^{\prime} \quad e \\
\text { const }=\left(p^{m i n}\right)^{\prime} Q p^{m i n}+c^{\prime} p^{m i n} .
\end{array}
$$

A constante definida em (5.23) pode ser desprezada na solução do modelo, pois não altera o problema de minimização.

Acrescentando as variáveis de folga $s_{\tilde{p}}^{k}, s_{\theta_{1}}^{k}$ e $s_{\theta_{2}}^{k}$, podemos escrever, finalmente, o problema primal de fluxo de potência ótimo DC utilizando o princípio do mínimo esforço: 


$$
\begin{array}{crl}
\operatorname{minimizar} \frac{\alpha}{2} \sum_{k=1}^{t}\left[\left(\theta^{k}\right)^{\prime} \tilde{B} \theta^{k}+c_{\theta}^{\prime} \theta^{k}\right]+\frac{\beta}{2} \sum_{k=1}^{t}\left[\left(\tilde{p}^{k}\right)^{\prime} Q \tilde{p}^{k}+c_{p}^{\prime} \tilde{p}^{k}\right] & \\
\text { sujeito a } & k=1, \cdots, t \\
B \theta^{k}-E \tilde{p}^{k}=\tilde{l}^{k}, & k=1, \cdots, t \\
U \theta^{k}+s_{\theta_{1}}^{k}=f^{\text {max }}, & k=1, \cdots, t \\
U \theta^{k}-s_{\theta_{2}}^{k}=f^{\text {min }}, & k=1, \cdots, t \\
\tilde{p}^{k}+s_{\tilde{p}}^{k}=\tilde{p}^{\text {max }}, & \\
\sum_{k=1}^{t} \tilde{p}^{k}=\tilde{q} & \\
\left(s_{\theta_{1}}^{k}, s_{\theta_{2}}^{k}, s_{\tilde{p}}^{k}, \tilde{p}^{k},\right) \geq 0, \quad \theta^{k} & \text { livre. }
\end{array}
$$

\subsection{Problema de Pré-Despacho Dual}

Se no problema de pré-despacho via princípio do mínimo esforço supormos que o vetor de injeções líquidas de potência ativa nas barras é livre, podemos associar à este vetor uma função a ser maximizada que incluirá o índice de mínimo esforço (OLIVEIRA; NEPOMUCENO; SOARES, 2003, 2005; OLIVEIRA; SOARES, 2000).

O objetivo desta seção é escrever o problema dual associado ao problema primal (5.24) a (5.30). Para isto, procederemos como segue:

1. Multiplicamos cada restrição (5.25) a (5.29), respectivamente, por $\left(y_{1}^{k}\right)^{\prime},\left(y_{2}^{k}\right)^{\prime},\left(y_{3}^{k}\right)^{\prime},\left(y_{4}^{k}\right)^{\prime}$ e $\left(y_{a}\right)^{\prime}$ e somamos membro a membro. A seguir, fatoramos a expressão do lado esquerdo em função de $\theta^{k}, \tilde{p}^{k}, s_{\theta_{1}}^{k}, s_{\theta_{2}}^{k}$ e $s_{\tilde{p}}^{k}$, obtendo:

$$
\begin{gathered}
{\left[\left(y_{1}^{k}\right)^{\prime} B+\left(y_{2}^{k}\right)^{\prime} U+\left(y_{3}^{k}\right)^{\prime} U\right] \theta^{k}+\left[-\left(y_{1}^{k}\right)^{\prime} E+\left(y_{4}^{k}\right)^{\prime}+\left(y_{a}\right)^{\prime}\right] \tilde{p}^{k}+\left(y_{2}^{k}\right)^{\prime} s_{\theta_{1}}^{k}-\left(y_{3}^{k}\right)^{\prime} s_{\theta_{2}}^{k}+\left(y_{4}^{k}\right)^{\prime} s_{\tilde{p}}^{k}=} \\
=\left(y_{1}^{k}\right)^{\prime} \tilde{l}^{k}+\left(y_{2}^{k}\right)^{\prime} f^{m a x}+\left(y_{3}^{k}\right)^{\prime} f^{m i n}+\left(y_{4}^{k}\right)^{\prime} p^{\max }+\left(y_{a}\right)^{\prime} \tilde{q} .
\end{gathered}
$$

Deste modo, o problema dual pode ser escrito como: 
$\operatorname{maximizar} \sum_{k=1}^{t}\left\{\left(\tilde{l}^{k}\right)^{\prime} y_{1}^{k}+\left(f^{\max }\right)^{\prime} y_{2}^{k}+\left(f^{\min }\right)^{\prime} y_{3}^{k}+\left(p^{\max }\right)^{\prime} y_{4}^{k}+(\tilde{q})^{\prime} y_{a}-\sum_{k=1}^{t}\left[\frac{\alpha}{2}\left(\theta^{k}\right)^{\prime} \tilde{B} \theta^{k}-\frac{\beta}{2}\left(\tilde{p}^{k}\right)^{\prime} Q \tilde{p}^{k}\right]\right\}$

sujeito a

$$
\begin{gathered}
B^{\prime} y_{1}^{k}+U^{\prime} y_{2}^{k}+U^{\prime} y_{3}^{k}=\tilde{B} \theta^{k}+c_{\theta}, \quad k=1,2, \cdots, t \\
-E^{\prime} y_{1}^{k}+y_{4}^{k}+y_{a} \leq Q \tilde{p}^{k}+c_{p}, \quad k=1,2, \cdots, t \\
y_{2}^{k} \leq 0, \quad k=1,2, \cdots, t \\
-y_{3}^{k} \leq 0, \quad k=1,2, \cdots, t \\
y_{4}^{k} \leq 0, \quad k=1,2, \cdots, t
\end{gathered}
$$

em que $\tilde{q}=q-\sum_{k=1}^{t} p^{\text {min }}$

2. Substituindo as variáveis $y_{1}^{k}, y_{2}^{k}, y_{3}^{k}$ e $y_{4}^{k}$, respectivamente, por $y_{\theta \tilde{p}}^{k},-w_{\theta_{1}}^{k}, w_{\theta_{2}}^{k}$ e $-w_{\tilde{p}}^{k}$, o problema anterior pode ser reescrito na seguinte forma:

$$
\begin{gathered}
\operatorname{maximizar} \sum_{k=1}^{t}\left\{\left(\tilde{l}^{k}\right)^{\prime} y_{\theta \tilde{p}}^{k}-\left(f^{\max }\right)^{\prime} w_{\theta_{1}}^{k}+\left(f^{m i n}\right)^{\prime} w_{\theta_{2}}^{k}-\left(\tilde{p}^{\max }\right)^{\prime} w_{\tilde{p}}^{k}+(\tilde{q})^{\prime} y_{a}-\frac{\alpha}{2}\left[\left(\theta^{k}\right)^{\prime} \tilde{B} \theta^{k}\right]-\frac{\beta}{2}\left[\left(\tilde{p}^{k}\right)^{\prime} Q \tilde{p}^{k}\right]\right\} \\
\qquad \begin{array}{c}
B^{\prime} y_{\theta \tilde{p}}^{k}-U^{\prime} w_{\theta_{1}}^{k}+U^{\prime} w_{\theta_{2}}^{k}-\tilde{B} \theta^{k}=c_{\theta} \\
-E^{\prime} y_{\theta \tilde{p}}^{k}-w_{\tilde{p}}^{k}+y_{a}-Q \tilde{p}^{k} \leq c_{p} \\
\text { sujeito a } w_{\theta_{1}}^{k} \geq 0 \\
w_{\theta_{2}}^{k} \geq 0 \\
w_{\tilde{p}}^{k} \geq 0 .
\end{array}
\end{gathered}
$$

Acrescentando a variável de folga $z_{\tilde{p}}^{k}$, obtemos o problema dual de fluxo em redes via princípio do mínimo esforço:

$$
\begin{gathered}
\operatorname{maximizar} \sum_{k=1}^{t}\left\{\left(\tilde{l}^{k}\right)^{\prime} y_{\theta \tilde{p}}^{k}-\left(f^{\max }\right)^{\prime} w_{\theta_{1}}^{k}+\left(f^{m i n}\right)^{\prime} w_{\theta_{2}}^{k}-\left(\tilde{p}^{\text {max }}\right)^{\prime} w_{\tilde{p}}^{k}+(\tilde{q})^{\prime} y_{a}-\right. \\
\left.\frac{\alpha}{2}\left[\left(\theta^{k}\right)^{\prime} \tilde{B} \theta^{k}\right]-\frac{\beta}{2}\left[\left(\tilde{p}^{k}\right)^{\prime} Q \tilde{p}^{k}\right]\right\} \\
\text { sujeito a } \quad B^{\prime} y_{\theta \tilde{p}}^{k}-U^{\prime} w_{\theta_{1}}^{k}+U^{\prime} w_{\theta_{2}}^{k}-\tilde{B} \theta^{k}=c_{\theta} \\
-E^{\prime} y_{\theta \tilde{p}}^{k}-w_{\tilde{p}}^{k}+y_{a}-Q \tilde{p}^{k}+z_{\tilde{p}}^{k}=c_{p} \\
\left(w_{\theta_{1}}^{k}, w_{\theta_{2}}^{k}, w_{\tilde{p}}^{k}, z_{\tilde{p}}^{k}\right) \geq 0, \quad y_{\theta \tilde{p}}^{k} \quad e \quad y_{a} \text { livres. }
\end{gathered}
$$




\subsection{Condições de Otimalidade}

As condições de otimalidade para os problemas primal e dual são dadas pelas factibilidades primal e dual e pelas condições de complementaridade (ALMEIDA, 87).

1. Factibilidade Primal

$$
\begin{array}{rlrl}
B \theta^{k}-E \tilde{p}^{k}=\tilde{l}^{k}, & k=1, \cdots, t \\
U \theta^{k}+s_{\theta_{1}}^{k}=f^{\text {max }}, & k=1, \cdots, t \\
U \theta^{k}-s_{\theta_{2}}^{k}=f^{\text {min }}, & k=1, \cdots, t \\
\tilde{p}^{k}+s_{\tilde{p}}^{k}=\tilde{p}^{\text {max }}, & k=1, \cdots, t \\
\sum_{k=1}^{t} \tilde{p}^{k}=\tilde{q} & & \\
\left(s_{\theta_{1}}^{k}, s_{\theta_{2}}^{k}, s_{\tilde{p}}^{k}, \tilde{p}^{k},\right) \geq 0, \quad \theta^{k} & \text { livre. } &
\end{array}
$$

2. Factibilidade Dual

$$
\begin{gathered}
B^{\prime} y_{\theta \tilde{p}}^{k}-U^{\prime} w_{\theta_{1}}^{k}+U^{\prime} w_{\theta_{2}}^{k}-\tilde{B} \theta^{k}=c_{\theta} \\
-E^{\prime} y_{\theta \tilde{p}}^{k}-w_{\tilde{p}}^{k}+y_{a}-Q \tilde{p}^{k}+z_{\tilde{p}}^{k}=c_{p} \\
\left(w_{\theta_{1}}^{k}, w_{\theta_{2}}^{k}, w_{\tilde{p}}^{k}, z_{\tilde{p}}^{k}\right) \geq 0, \quad y_{\theta \tilde{p}}^{k} \quad e \quad y_{a} \quad \text { livres. }
\end{gathered}
$$

3. Condições de Complementaridade

$$
\begin{aligned}
S_{\theta_{1}}^{k} W_{\theta_{1}}^{k} e & =0 \\
S_{\theta_{2}}^{k} W_{\theta_{2}}^{k} e & =0 \\
S_{\tilde{p}}^{k} W_{\tilde{p}}^{k} e & =0 \\
\tilde{P}^{k} Z_{\tilde{p}}^{k} e & =0 .
\end{aligned}
$$

\subsection{Método de Newton}

Aplicando o método de Newton (Apêndice A) às condições de otimalidade (5.36) a (5.46), obtemos o seguinte sistema linear:

$$
J d=r
$$


em que $J$ representa a matriz Jacobiana dada por:

$$
\left[\begin{array}{rrrrr}
M_{1} & 0 & 0 & \cdots & 0 \\
0 & M_{2} & 0 & \cdots & 0 \\
0 & 0 & M_{3} & \cdots & 0 \\
\vdots & \vdots & \ddots & \vdots & \vdots \\
0 & 0 & 0 & \cdots & M_{k} \\
I_{\tilde{p}^{1}} & I_{\tilde{p}^{2}} & I_{\tilde{p}^{3}} & \cdots & I_{\tilde{p}^{k}}
\end{array}\right]
$$

em que cada $M_{k}$ corresponde à submatriz

$$
\left[\begin{array}{rrrrrrrrrrr}
B & -E & 0 & 0 & 0 & 0 & 0 & 0 & 0 & 0 & 0 \\
U & 0 & I & 0 & 0 & 0 & 0 & 0 & 0 & 0 & 0 \\
U & 0 & 0 & -I & 0 & 0 & 0 & 0 & 0 & 0 & 0 \\
0 & I & 0 & 0 & I & 0 & 0 & 0 & 0 & 0 & 0 \\
-B & 0 & 0 & 0 & 0 & B^{\prime} & -U^{\prime} & U^{\prime} & 0 & 0 & 0 \\
0 & -Q & 0 & 0 & 0 & -E^{\prime} & 0 & 0 & -I & I & I \\
0 & 0 & W_{\theta_{1}}^{k} & 0 & 0 & 0 & S_{\theta_{1}}^{k} & 0 & 0 & 0 & 0 \\
0 & 0 & 0 & W_{\theta_{2}}^{k} & 0 & 0 & 0 & S_{\theta_{2}}^{k} & 0 & 0 & 0 \\
0 & 0 & 0 & 0 & W_{\tilde{p}}^{k} & 0 & 0 & 0 & S_{\tilde{p}}^{k} & 0 & 0 \\
0 & Z_{\tilde{p}}^{k} & 0 & 0 & 0 & 0 & 0 & 0 & 0 & \tilde{P}^{k} & 0
\end{array}\right],
$$

cada $I_{\tilde{p}^{k}}$ corresponde à matriz

$$
I_{\tilde{p}^{k}}^{\prime}=\left[\begin{array}{lllllllllll}
0 & I & 0 & 0 & 0 & 0 & 0 & 0 & 0 & 0 & 0
\end{array}\right],
$$

$d$ representa as direções de Newton associadas às variáveis do problema:

$$
d^{\prime}=\left[\begin{array}{lllllllllll}
d \theta^{k} & d \tilde{p}^{k} & d s_{\theta_{1}}^{k} & d s_{\theta_{2}}^{k} & d s_{\tilde{p}}^{k} & d y_{\theta \tilde{p}}^{k} & d w_{\theta_{1}}^{k} & d w_{\theta_{2}}^{k} & d w_{\tilde{p}}^{k} & d z_{\tilde{p}}^{k} & d y_{a}
\end{array}\right]
$$

e $r$ representa o vetor dos resíduos, cujas componentes são dadas por: 


$$
\begin{aligned}
r_{l} & =\tilde{l}^{k}-B \theta^{k}+E \tilde{p}^{k} \\
r_{t 1} & =f^{m a x}-U \theta^{k}-s_{\theta_{1}}^{k} \\
r_{t 2} & =f^{m i n}-U \theta^{k}+s_{\theta_{2}}^{k} \\
r_{p} & =\tilde{p}^{m a x}-\tilde{p}^{k}-s_{\tilde{p}}^{k} \\
r_{y} & =c_{\theta}-B^{\prime} y_{\theta \tilde{p}}^{k}+U^{\prime} w_{\theta_{1}}^{k}-U^{\prime} w_{\theta_{2}}^{k}+\tilde{B} \theta^{k} \\
r_{g} & =c_{p}+E^{\prime} y_{\theta \tilde{p}}^{k}+w_{\tilde{p}}^{k}-y_{a}-z_{\tilde{p}}^{k}+Q \tilde{p}^{k} \\
r_{z p} & =\mu e-\tilde{P}^{k} Z_{\tilde{p}}^{k} e \\
r_{w p} & =\mu e-S_{\tilde{p}}^{k} W_{\tilde{p}}^{k} e \\
r_{s 1} & =\mu e-S_{\theta_{1}}^{k} W_{\theta_{1}}^{k} e \\
r_{s 2} & =\mu e-S_{\theta_{2}}^{k} W_{\theta_{2}}^{k} e \\
r_{m} & =\tilde{q}-\sum_{k=1}^{t} \tilde{p}^{k} .
\end{aligned}
$$

Portanto, as direções de Newton são dadas pelo seguinte sistema linear:

$$
\begin{aligned}
B d \theta^{k}-E d \tilde{p}^{k} & =r_{l} \\
U d \theta^{k}+d s_{\theta_{1}}^{k} & =r_{t 1} \\
U d \theta^{k}-d s_{\theta_{2}}^{k} & =r_{t 2} \\
d \tilde{p}^{k}+d s_{\tilde{p}}^{k} & =r_{p} \\
B^{\prime} d y_{\theta \tilde{p}}^{k}-U^{\prime} d w_{\theta_{1}}^{k}+U^{\prime} d w_{\theta_{2}}^{k}-\tilde{B} d \theta^{k} & =r_{y} \\
-E^{\prime} d y_{\theta \tilde{p}}^{k}-d w_{\tilde{p}}^{k}+d y_{a}+d z_{\tilde{p}}^{k}-Q d \tilde{p}^{k} & =r_{g} \\
Z_{\tilde{p}}^{k} d \tilde{p}^{k}+\tilde{P}^{k} d z_{\tilde{p}}^{k} & =r_{z p} \\
W_{\tilde{p}}^{k} d s_{\tilde{p}}^{k}+S_{\tilde{p}}^{k} d w_{\tilde{p}}^{k} & =r_{w p} \\
W_{\theta_{1}}^{k} d s_{\theta_{1}}^{k}+S_{\theta_{1}}^{k} d w_{\theta_{1}}^{k} & =r_{s 1} \\
W_{\theta_{2}}^{k} d s_{\theta_{2}}^{k}+S_{\theta_{2}}^{k} d w_{\theta_{1}}^{k} & =r_{s 2} \\
\sum_{k=1}^{t} d \tilde{p}^{k} & =r_{m} .
\end{aligned}
$$

Nosso próximo passo consistirá em resolver o sistema constituído pelas Equações 
(5.47) a (5.57). Para isto, utilizaremos o conceito de substituição de variáveis com o objetivo de reduzir a dimensão do sistema linear.

\subsection{Redução do Sistema Linear}

Eliminando as variáveis $d z_{\tilde{p}}^{k}, d w_{\tilde{p}}^{k}, d w_{\theta_{1}}^{k}, d w_{\theta_{2}}^{k}, d s_{\theta_{1}}^{k}, d s_{\theta_{2}}^{k}$ e $d s_{\tilde{p}}^{k}$, respectivamente, nas Equações (5.53), (5.54), (5.55), (5.56), (5.48), (5.49) e (5.50), temos:

$$
\begin{aligned}
d z_{\tilde{p}}^{k} & =\left(\tilde{P}^{k}\right)^{-1}\left[r_{z p}-Z_{\tilde{p}}^{k} d \tilde{p}^{k}\right] \\
d w_{\tilde{p}}^{k} & =\left(S_{\tilde{p}}^{k}\right)^{-1}\left[r_{w p}-W_{\tilde{p}}^{k} d s_{\tilde{p}}^{k}\right] \\
d w_{\theta_{1}}^{k} & =\left(S_{\theta_{1}}^{k}\right)^{-1}\left[r_{s 1}-W_{\theta_{1}}^{k} d s_{\theta_{1}}^{k}\right] \\
d w_{\theta_{2}}^{k} & =\left(S_{\theta_{2}}^{k}\right)^{-1}\left[r_{s 2}-W_{\theta_{2}}^{k} d s_{\theta_{2}}^{k}\right] \\
d s_{\theta_{1}}^{k} & =r_{t 1}-U d \theta^{k} \\
d s_{\theta_{2}}^{k} & =-r_{t 2}+U d \theta^{k} \\
d s_{\tilde{p}}^{k} & =r_{p}-d \tilde{p}^{k} .
\end{aligned}
$$

Substituindo (5.62) e (5.63), respectivamente, em (5.60) e (5.61), obtemos:

$$
\begin{aligned}
& d w_{\theta_{1}}^{k}=\left(S_{\theta_{1}}^{k}\right)^{-1}\left[r_{s 1}-W_{\theta_{1}}^{k} r_{t 1}+W_{\theta_{1}}^{k} U d \theta^{k}\right] \\
& d w_{\theta_{2}}^{k}=\left(S_{\theta_{2}}^{k}\right)^{-1}\left[r_{s 2}+W_{\theta_{2}}^{k} r_{t 2}-W_{\theta_{2}}^{k} U d \theta^{k}\right]
\end{aligned}
$$

Substituindo (5.65) e (5.66) em (5.51) e reorganizando os termos, temos:

$$
B^{\prime} d y_{\theta \tilde{p}}^{k}-\hat{B}^{k} d \theta^{k}=r_{a}
$$

em que

$$
\begin{aligned}
\hat{B}^{k} & =U^{\prime} D_{\theta_{1}}^{k} U+U^{\prime} D_{\theta_{2}}^{k} U+\tilde{B} \\
D_{\theta_{1}}^{k} & =\left(S_{\theta_{1}}^{k}\right)^{-1} W_{\theta_{1}}^{k} \\
D_{\theta_{2}}^{k} & =\left(S_{\theta_{2}}^{k}\right)^{-1} W_{\theta_{2}}^{k} \\
r_{a} & =r_{y}+U^{\prime}\left(S_{\theta_{1}}^{k}\right)^{-1} r_{s 1}-U^{\prime} D_{\theta_{1}}^{k} r_{t 1}-U^{\prime}\left(S_{\theta_{2}}^{k}\right)^{-1} r_{s 2}-U^{\prime} D_{\theta_{2}}^{k} r_{t 2} .
\end{aligned}
$$


Substituindo (5.64) em (5.59), obtemos:

$$
d w_{\tilde{p}}^{k}=\left(S_{\tilde{p}}^{k}\right)^{-1}\left[r_{w p}-W_{\tilde{p}}^{k} r_{p}+W_{\tilde{p}}^{k} d \tilde{p}^{k}\right]
$$

Substituindo (5.68) e (5.58) na Equação (5.52) e reorganizando os termos, temos:

$$
-E^{\prime} d y_{\theta \tilde{p}}^{k}-D_{\tilde{p}}^{k} d \tilde{p}^{k}+d y_{a}=r_{b}
$$

em que

$$
\begin{aligned}
D_{\tilde{p}}^{k} & =\left(S_{\tilde{p}}^{k}\right)^{-1} W_{\tilde{p}}^{k}+\left(\tilde{P}^{k}\right)^{-1} Z_{\tilde{p}}^{k}+Q \\
r_{b} & =r_{g}+\left(S_{\tilde{p}}^{k}\right)^{-1} r_{w p}-\left(S_{\tilde{p}}^{k}\right)^{-1} W_{\tilde{p}}^{k} r_{p}-\left(\tilde{P}^{k}\right)^{-1} r_{z p}
\end{aligned}
$$

Com estas simplificações, o sistema original (Equações (5.47) a (5.57)) fica reduzido ao seguinte conjunto de equações:

$$
\begin{aligned}
B d \theta^{k}-E d \tilde{p}^{k} & =r_{l} \\
B^{\prime} d y_{\theta \tilde{p}}^{k}-\hat{B}^{k} d \theta^{k} & =r_{a} \\
-E^{\prime} d y_{\theta \tilde{p}}^{k}-D_{\tilde{p}}^{k} d \tilde{p}^{k}+d y_{a} & =r_{b} \\
\sum_{k=1}^{t} d \tilde{p}^{k} & =r_{m} .
\end{aligned}
$$

Observemos que $D_{\tilde{p}}^{k}$ é uma matriz diagonal de orgem $g$, enquanto que a matriz $\hat{B}^{k}$, possui a seguinte estrutura:

$\hat{B}^{k}=U^{\prime} D_{\theta_{1}}^{k} U+U^{\prime} D_{\theta_{2}}^{k} U+\tilde{B}=U^{\prime} D_{\theta_{1}}^{k} U+U^{\prime} D_{\theta_{2}}^{k} U+U^{\prime} R U=U^{\prime}\left(D_{\theta_{1}}^{k}+D_{\theta_{2}}^{k}+R\right) U=U^{\prime} D U$

Portanto, a matriz $\hat{B}^{k}$ que aparece na Equação (5.71) não é diagonal, tem dimensão $m \times m$, enquanto que $D$ tem dimensão $n \times n$.

Vimos na Seção (2.4) que a matriz $\tilde{B}=U^{\prime} R U$ que aparece na função objetivo do problema primal e a matriz $B=A U$ são singulares e só dependem dos parâmetros da rede. No Apêncide B mostramos que se eliminarmos uma linha e uma coluna destas matrizes, as matrizes resultantes serão invertíveis. Na Equação (5.71), a matriz $\hat{B}^{k}$ possui, 
por construção, as mesmas propriedades que as matrizes $B$ e $\tilde{B}$. Assim, ela também é singular.

Para continuar com a redução do sistema, vamos eliminar uma barra $j$ do sistema adotando-a como referência angular $\left(\theta_{j}=0\right)$. Isto é equivalente a eliminar a j-ésima linha e a j-ésima coluna das matrizes $B$ e $\hat{B}^{k}$.

Assim, considerando que o sistema composto pelas Equações (5.70) a (5.73) está redimensionado conforme as observações anteriores, podemos considerar, a partir de agora, duas formas de eliminação de variáveis, com a finalidade de verificar se há alguma vantagem da implementação de uma delas em relação à implementação da outra.

1. Eliminação de variáveis 1 (E1)

Eliminando $d \theta^{k}$ na Equação (5.70) e $d y_{\theta \tilde{p}}^{k}$ na Equação (5.71), temos:

$$
\begin{aligned}
d \theta^{k} & =B^{-1}\left[r_{l}+E d \tilde{p}^{k}\right] \\
d y_{\theta \tilde{p}}^{k} & =\left(B^{\prime}\right)^{-1}\left[r_{a}+\hat{B}^{k} d \theta^{k}\right] .
\end{aligned}
$$

Substituindo (5.74) em (5.75) e reorganizando os termos, podemos reescrever $d y_{\theta \tilde{p}}^{k}$ na forma:

$$
d y_{\theta \tilde{p}}^{k}=\left[\left(B^{\prime}\right)^{-1} \hat{B}^{k} B^{-1} E\right] d \tilde{p}^{k}+\left(B^{\prime}\right)^{-1}\left[r_{a}+\hat{B}^{k} B^{-1} r_{l}\right]
$$

Substituindo (5.76) na Equação (5.72) e reorganizando os termos, obtemos:

$$
-\left[W^{\prime} \hat{B}^{k} W+D_{\tilde{p}}^{k}\right] d \tilde{p}^{k}+d y_{a}=r_{b}+W^{\prime} r_{a}+W^{\prime} \hat{B}^{k} B^{-1} r_{l}
$$

em que estamos definindo

$$
W=B^{-1} E .
$$

Lembrando que $\hat{B}^{k}=U^{\prime} D U$ e definindo

$$
Z=W^{\prime} U^{\prime}
$$


a Equação (5.77) pode ser reescrita como:

$$
-\left[Z D Z^{\prime}+D_{\tilde{p}}^{k}\right] d \tilde{p}^{k}+d y_{a}=r
$$

em que

$$
r=r_{b}+W^{\prime} r_{a}+Z D B^{-1} r_{l}
$$

Portanto, o conjunto de Equações (5.70) a (5.73) fica reduzido às seguintes equações:

$$
\begin{aligned}
-\left[Z D Z^{\prime}+D_{\tilde{p}}^{k}\right] d \tilde{p}^{k}+d y_{a} & =r \\
\sum_{k=1}^{t} d \tilde{p}^{k} & =r_{m}
\end{aligned}
$$

Eliminando $d \tilde{p}^{k}$ na Equação (5.79), temos:

$$
d \tilde{p}^{k}=\left[Z D Z^{\prime}+D_{\tilde{p}}^{k}\right]^{-1}\left[-r+d y_{a}\right]
$$

Substituindo (5.81) em (5.80) e reorganizando os termos, obtemos finalmente:

$$
\sum_{k=1}^{t}\left[Z D Z^{\prime}+D_{\tilde{p}}^{k}\right]^{-1} d y_{a}=r_{m}+\sum_{k=1}^{t}\left[Z D Z^{\prime}+D_{\tilde{p}}^{k}\right]^{-1} r
$$

Nesta equação, a matriz $M=Z D Z^{\prime}+D_{\tilde{p}}^{k}$ tem dimensão correspondente ao número de geradores do sistema.

A Equação (5.82) foi implementada no Matlab, versão 5.3, utilizando a decomposição de Cholesky para calcular a inversa de $M$, como uma função intrínseca ao código.

Uma implementação alternativa foi feita sem utilizar a função intrínseca do Matlab, para calcular a inversa de $M$.

Neste caso, utilizamos a fórmula de Shermann-Morrison-Woodbury (DUFF; ERISMAN; REID, 1986), cuja forma é: 


$$
\left(C+\bar{U} S V^{\prime}\right)^{-1}=C^{-1}-C^{-1} \bar{U}\left(S^{-1}+V^{\prime} C^{-1} \bar{U}\right)^{-1} V^{\prime} C^{-1}
$$

em que, $\bar{U}$ e $V^{\prime}$ são matrizes de ordem $p \times q$ e $S$ é uma matriz de ordem $q \times q$, geralmente com $p<<q$.

Para aplicar esta fórmula no cálculo da inversa de $M$, sejam

$$
\bar{U} \equiv Z, \quad V^{\prime} \equiv \bar{U}^{\prime}, \quad S \equiv D \quad \text { e } \quad C \equiv D_{\tilde{p}}^{k}
$$

Com esta notação e tendo em vista a Equação (5.83) a inversa de $M$ é dada por:

$$
\begin{aligned}
{\left[Z D Z^{\prime}+D_{\tilde{p}}^{k}\right]^{-1} } & =\left(D_{\tilde{p}}^{k}\right)^{-1}-\left(D_{\tilde{p}}^{k}\right)^{-1} Z\left[(D I A G)^{-1}+Z^{\prime}\left(D_{\tilde{p}}^{k}\right)^{-1} Z\right]^{-1} Z^{\prime}\left(D_{\tilde{p}}^{k}\right)^{-1} \\
& =\left(D_{\tilde{p}}^{k}\right)^{-1}-\left(D_{\tilde{p}}^{k}\right)^{-1} Z H^{-1} Z^{\prime}\left(D_{\tilde{p}}^{k}\right)^{-1}
\end{aligned}
$$

em que

$$
H=(D)^{-1}+Z^{\prime}\left(D_{\tilde{p}}^{k}\right)^{-1} Z
$$

Substituindo (5.84) em (5.81), temos:

$$
d \tilde{p}^{k}=\left\{\left(D_{\tilde{p}}^{k}\right)^{-1}-\left(D_{\tilde{p}}^{k}\right)^{-1} Z H^{-1} Z^{\prime}\left(D_{\tilde{p}}^{k}\right)^{-1}\right\}\left[-r+d y_{a}\right]
$$

Agora, substituindo (5.85) em (5.80) e reorganizando os termos, obtemos:

$$
\sum_{k=1}^{t}\left[\left(D_{\tilde{p}}^{k}\right)^{-1}-\left(D_{\tilde{p}}^{k}\right)^{-1} Z H^{-1} Z^{\prime}\left(D_{\tilde{p}}^{k}\right)^{-1}\right] d y_{a}=r_{m}+\sum_{k=1}^{t}\left[\left(D_{\tilde{p}}^{k}\right)^{-1}-\left(D_{\tilde{p}}^{k}\right)^{-1} Z H^{-1} Z^{\prime}\left(D_{\tilde{p}}^{k}\right)^{-1}\right] r
$$

2. Eliminação de variáveis 2 (E2)

Eliminando $d \theta^{k}$ na Equação (5.71) e $d \tilde{p}^{k}$ na Equação (5.72), temos:

$$
\begin{aligned}
d \theta^{k} & =\left(\hat{B}^{k}\right)^{-1}\left[-r_{a}+B^{\prime} d y_{\theta \tilde{p}}^{k}\right] \\
d \tilde{p}^{k} & =\left(D_{\tilde{p}}^{k}\right)^{-1}\left[-r_{b}-E^{\prime} d y_{\theta \tilde{p}}^{k}+d y_{a}\right]
\end{aligned}
$$


Substituindo estas duas equações em (5.70) e reorganizando os termos, obtemos:

$$
\left[B\left(\hat{B}^{k}\right)^{-1} B^{\prime}+E\left(D_{\tilde{p}}^{k}\right)^{-1} E^{\prime}\right] d y_{\theta \tilde{p}}^{k}-E\left(D_{\tilde{p}}^{k}\right)^{-1} d y_{a}=r
$$

em que

$$
r=r_{l}+B\left(\hat{B}^{k}\right)^{-1} r_{a}-E\left(D_{\tilde{p}}^{k}\right)^{-1} r_{b}
$$

Portanto, o conjunto de Equações (5.70) a (5.73) fica reduzido às seguintes equações:

$$
\begin{aligned}
{\left[B\left(\hat{B}^{k}\right)^{-1} B^{\prime}+E\left(D_{\tilde{p}}^{k}\right)^{-1} E^{\prime}\right] d y_{\theta \tilde{p}}^{k}-E\left(D_{\tilde{p}}^{k}\right)^{-1} d y_{a} } & =r \\
\sum_{k=1}^{t}\left(D_{\tilde{p}}^{k}\right)^{-1}\left[-r_{b}-E^{\prime} d y_{\theta \tilde{p}}^{k}+d y_{a}\right] & =r_{m}
\end{aligned}
$$

em que a Equação (5.88) foi utilizada para escrever (5.91).

Eliminando $d y_{\theta \tilde{p}}^{k}$ em (5.90), temos:

$$
d y_{\theta \tilde{p}}^{k}=\left[B\left(\hat{B}^{k}\right)^{-1} B^{\prime}+E\left(D_{\tilde{p}}^{k}\right)^{-1} E^{\prime}\right]^{-1}\left[r+E\left(D_{\tilde{p}}^{k}\right)^{-1} d y_{a}\right]
$$

Substituindo esta última equação em (5.91) e reorganizando os termos, obtemos, finalmente:

$$
\sum_{k=1}^{t}\left[\left(D_{\tilde{p}}^{k}\right)^{-1}-\left(D_{\tilde{p}}^{k}\right)^{-1} E^{\prime} M^{-1} E\left(D_{\tilde{p}}^{k}\right)^{-1}\right] d y_{a}=r_{m}+\sum_{k=1}^{t}\left(D_{\tilde{p}}^{k}\right)^{-1}\left[r_{b}+E^{\prime} M^{1} r\right]
$$

em que

$$
M=B\left(\hat{B}^{k}\right)^{-1} B^{\prime}+E\left(D_{\tilde{p}}^{k}\right)^{-1} E^{\prime}
$$

Na Equação (5.93) temos que calcular inicialmente e para cada tempo, a inversa da matriz $M$ que tem dimensão equivalente ao número de barras do sistema. Isto leva a um esforço computacional considerável para sistemas de grande porte. 
A despeito disso, fizemos a implementação computacional desta equação no Matlab, e utilizando sua função intrínseca para o cálculo das inversas das matrizes.

Alternativamente, fizemos também uma implementação da Equação (5.93), mas calculando a inversa de $M$ utilizando a fórmula (5.84) de SMW.

Definindo,

$$
\bar{U}=E, \quad S=\left(D_{\tilde{p}}^{k}\right)^{-1}, \quad V^{\prime}=E \quad \text { e } \quad C=B\left(\hat{B}^{k}\right)^{-1} B^{\prime},
$$

a inversa de $M$ é dada por:

$$
\left[B\left(\hat{B}^{k}\right)^{-1} B^{\prime}+E\left(D_{\tilde{p}}^{k}\right)^{-1} E^{\prime}\right]^{-1}=\left(B^{\prime}\right)^{-1} \hat{B}^{k} B^{-1}-\left(B^{\prime}\right)^{-1} \hat{B}^{k} W\left(D_{\tilde{p}}^{k}+W^{\prime} \hat{B}^{k} W\right)^{-1} W^{\prime} \hat{B}^{k} B^{-1}
$$

em que utilizamos a definição anterior de $W=B^{-1} E$.

Substituindo (5.94) em (5.93), obtemos finalmente:

$$
\sum_{k=1}^{t}\left[\left(D_{\tilde{p}}^{k}\right)^{-1}-\left(D_{\tilde{p}}^{k}\right)^{-1} E^{\prime} N E\left(D_{\tilde{p}}^{k}\right)^{-1}\right] d y_{a}=r_{m}+\sum_{k=1}^{t}\left(D_{\tilde{p}}^{k}\right)^{-1}\left[r_{b}+E^{\prime} N r\right]
$$

em que

$$
\begin{aligned}
N & =\left(B^{\prime}\right)^{-1} \hat{B}^{k} B^{-1}-\left(B^{\prime}\right)^{-1} \hat{B}^{k} W G^{-1} W^{\prime} \hat{B}^{k} B^{-1}, \\
G & =D_{\tilde{p}}^{k}+W^{\prime} \hat{B}^{k} W .
\end{aligned}
$$

\subsection{Métodos Primal-Dual e Preditor-Corretor}

Definindo os vetores $x=\cup_{i=1}^{t}\left(s_{\theta_{1}}^{k}, s_{\theta_{2}}^{k}, s_{\tilde{p}}^{k}, \tilde{p}^{k}\right), y=\cup_{k=1}^{t}\left(\theta^{k}, y_{\theta \tilde{p}}^{k}\right)$ e $z=\cup_{k=1}^{t}\left(w_{\theta_{1}}^{k}, w_{\theta_{2}}^{k}, w_{\tilde{p}}^{k}, z_{\tilde{p}}^{k}\right)$, a aplicação do método de Newton às condições de otimalidade resulta nos métodos, Primal-Dual via Princípio do Mínimo Esforço (PDPME) e Preditor-Corretor via Princípio do Mínimo Esforço (PCPME):

\section{Método PDPME}

Dados $\xi \in(0,1), \sigma=\frac{1}{n p \sqrt{n p}}, \quad n p=2 t(n+g)$; 
Escolha $\left(x^{0}, y^{0}, z^{0}\right) \in N_{-\infty}(\xi), \operatorname{com}\left(x^{0}, z^{0}\right)>0, \theta^{0}=0, y^{0}=0 \quad e \quad y_{a}^{0}=0$

Para $k=0,1,2, \ldots$

Escolha $\sigma^{k}=\sigma$;

Faça $\mu^{k}=\sigma^{k}\left(\frac{\gamma^{k}}{n p^{2} \sqrt{n p}}\right)$, se $\gamma^{k} \geq 1$ e $\mu^{k}=\sigma^{k}\left(\frac{\left(\gamma^{k}\right)^{2}}{n p}\right)$, se $\gamma^{k}<1, \gamma^{k}=\left(x^{k}\right)^{\prime} z^{k}$.

Calcule as direções de Newton $\Delta x^{k}, \Delta y^{k}, \Delta z^{k}$ e $\Delta y_{a}^{k}$.

Calcule $\alpha_{p}^{k}=\frac{-\tau}{\min _{i}\left(\frac{\Delta x_{i}^{k}}{x^{k}}\right)}$ e $\alpha_{d}^{k}=\frac{-\tau}{\min _{i}\left(\frac{\Delta z_{i}^{k}}{z_{i}^{k}}\right)}, \tau \in(0,1)$.

Faça $\alpha^{k}=\min \left(1, \alpha_{p}^{k}, \alpha_{d}^{k}\right)$, tal que $\left(x^{k}, y^{k}, z^{k}, y_{a}^{k}\right) \in N_{-\infty}(\xi)$;

Faça $\left(x^{k+1}, y^{k+1}, z^{k+1}, y_{a}^{k+1}\right)=\left(x^{k}, y^{k}, z^{k}, y_{a}^{k}\right)+\alpha^{k}\left(\Delta x^{k}, \Delta y^{k}, \Delta z^{k}, \Delta y_{a}^{k}\right)$

FIM.

\section{Método PCPME}

Dados $\xi \in(0,1), \quad n=2 t(n+g)$;

Escolha $\left(x^{0}, y^{0}, z^{0}\right) \in N_{-\infty}(\xi), \operatorname{com}\left(x^{0}, z^{0}\right)>0, \theta^{0}=0, y^{0}=0 \quad e \quad y_{a}^{0}=0$.

Para $k=0,1,2, \ldots$

(Passo Preditor)

Faça $\sigma^{k}=0$; e calcule as direções de Newton $\Delta x^{k}, \Delta y^{k}, \Delta z^{k}$ e $\Delta y_{a}^{k}$.

Calcule $\alpha_{p}^{k}=\frac{-\tau}{\min _{i}\left(\frac{\Delta x_{i}^{k}}{x_{i}^{k}}\right)}$ e $\alpha_{d}^{k}=\frac{-\tau}{\min _{i}\left(\frac{\Delta z_{i}^{k}}{z_{i}^{k}}\right)}, \tau \in(0,1)$.

Faça $\alpha^{k}=\min \left(1, \alpha_{p}^{k}, \alpha_{d}^{k}\right)$ tal que $\left(x^{k}, y^{k}, z^{k}, y_{a}^{k}\right) \in N_{-\infty}(\xi)$;

(Passo Corretor)

Faça $\sigma^{k}=1$; e calcule as direções de Newton $\Delta x^{k}, \Delta y^{k}, \Delta z^{k}$ e $\Delta y_{a}^{k}$.

Calcule $\alpha_{p}^{k}=\frac{-\tau}{\min _{i}\left(\frac{\Delta x_{i}^{k}}{x_{i}^{k}}\right)}$ e $\alpha_{d}^{k}=\frac{-\tau}{\min _{i}\left(\frac{\Delta z_{i}^{k}}{z_{i}^{k}}\right)}, \tau \in(0,1)$.

Faça $\alpha^{k}=\min \left(1, \alpha_{p}^{k}, \alpha_{d}^{k}\right)$;

Faça $\left(x^{k+1}, y^{k+1}, z^{k+1}, y_{a}^{k+1}\right)=\left(x^{k}, y^{k}, z^{k}, y_{a}^{k}\right)+\alpha^{k}\left(\Delta x^{k}, \Delta y^{k}, \Delta z^{k}, \Delta y_{a}^{k}\right)$

FIM.

\subsection{Modificação no Método PDPME}

É possível reorganizar o sistema (5.47) a (5.56) de tal modo que se possa reescrevê-lo na forma: 


$$
\begin{aligned}
A \Delta x & =r_{p} \\
Y \Delta y+B \Delta z & =r_{d} \\
S \Delta x+Z \Delta z & =\mu e-Z S e .
\end{aligned}
$$

em que $S$ e $Z$ são matrizes diagonais com elementos positivos na diagonal.

Em um método de ponto interior primal-dual a principal operação a ser realizada em cada iteração consiste na solução de um sistema semelhante a este. Em métodos como PCPME (tipo Mehrotra), precisamos resolver um sistema desta forma mais de uma vez em cada iteração, com a mesma matriz, mas com o lado direito diferente. A solução eficiente deste sistema é crítica, pois, em geral, sua matriz é de grande porte e esparsa exigindo técnicas sofisticadas para sua redução.

Felizmente, o Matlab incorpora excelentes pacotes para solução de sistemas com este tipo de matriz, tal que é possível resolver um problema como o proposto neste trabalho, por meio de um método de ponto interior, fazendo a redução do sistema como mostrado na Seção 5.7 e utilizando os comandos do Matlab.

Nesta Seção apresentamos uma modificação do Método PDPME, denominada versão perturbada do método primal-dual (VPMPD) cuja descrição é dada a seguir.

Consideremos a seguinte forma modificada do sistema não linear (5.96),

$$
\begin{aligned}
A \Delta x & =r_{p} \\
Y \Delta y+B \Delta z & =r_{d} \\
S \Delta x+Z \Delta z & =\sigma \mu e-Z S e+v e
\end{aligned}
$$

em que $\sigma$ é o parâmetro de centragem e $v$ é um parâmetro de perturbação, escolhido com a finalidade de aperfeiçoar a proximidade, em cada iteração $k$, da seqüência de soluções $\left(x^{k}, y^{k}, z^{k}\right)$ ao caminho central (POTRA; WRIGHT, 2000).

Sabemos que a grande diferença entre os métodos primais-duais está na escolha do ponto inicial, do parâmetro de centragem $\sigma$ e do tamanho do passo $\alpha$. Neste caso, o parâmetro de perturbação $v$ também é um fator determinante nesta diferença. 
Portanto, a forma como definiremos este parâmetro de perturbação $v$ influenciará o desempenho do método. O nosso objetivo é melhorar este desempenho com relação ao número de iterações. Assim, a nossa escolha para $v$ deve ser tal que permita um aperfeiçoamento, em cada iteração $k$, da proximidade da seqüência de soluções $\left(x^{k}, y^{k}, z^{k}\right)$ ao caminho central $C$ (POTRA; WRIGHT, 2000).

A nossa proposta, então, é que $v$ seja escolhido como o produto do parâmetro de centro $\sigma$ e a complementaridade $Z S$, isto é,

$$
v^{k}=\sigma^{k} Z^{k} S^{k} e, \quad k=0,1,2, \cdots
$$

em que o parâmetro de centro $\sigma$ pode ser definido de forma adaptativa como:

$$
\sigma^{k}=\frac{\mu^{k+1}}{\mu^{k}} .
$$

Assim, com uma escolha conveniente de um ponto inicial infactível, apresentamos a estrutura básica do método primal-dual vetor de perturbação (VPMPD) como segue:

\section{Método VPMPD}

Dados $\xi \in(0,1), \sigma^{0}=\frac{1}{n p \sqrt{n p}}, n p=2 t(n+g)$ e $\phi=\sigma^{0}$;

Escolha $\left(x^{0}, y^{0}, z^{0}\right) \in N_{-\infty}(\xi),\left(x^{0}, z^{0}\right)>0, \theta^{0}=0, \quad y_{a}^{0}=0 ;$

Faça $\gamma^{0}=\left(x^{0}\right)^{\prime} z^{0}, \mu^{0}=\frac{\gamma^{0}}{n p}$;

Para $k=0,1,2, \cdots$

Faça $\mu^{k+1}=\gamma^{k} \phi^{2}$, se $\gamma^{k} \geq 1$ ou $\mu^{k+1}=n p\left(\gamma^{k}\right)^{2} \phi^{2}$, se $\gamma^{k}<1$;

Faça $v^{k}=\sigma^{k} x^{k} z^{k}$

Faça $\sigma^{k+1}=\frac{\mu^{k+1}}{\mu^{k}}$;

Resolva (5.97) para obter $\left(\Delta x^{k}, \Delta y^{k}, \Delta z^{k}\right)$;

Calcule $\alpha_{p}^{k}=\frac{-\tau}{\min _{i}\left(\frac{\Delta x_{i}^{k}}{x_{i}^{k}}\right)}$ e $\alpha_{d}^{k}=\frac{-\tau}{\min _{i}\left(\frac{\Delta z_{i}^{k}}{z_{i}^{k}}\right)}, \tau \in(0,1)$;

Faça $\alpha^{k}=\min \left(1, \alpha_{p}^{k}, \alpha_{d}^{k}\right)$, tal que

$\left(x^{k}, y^{k}, z^{k}\right) \in N_{-\infty}(\xi)$

Faça $\left(x^{k+1}, y^{k+1}, z^{k+1}\right)=\left(x^{k}, y^{k}, z^{k}\right)+\alpha^{k}\left(\Delta x^{k}, \Delta y^{k}, \Delta z^{k}\right)$;

FIM. 
Este método tem um desempenho prático excelente como mostram os resultados apresentados no capítulo 6. Não fizemos uma análise da convergência do mesmo nem tão pouco de sua complexidade computacional, que serão objetos de proposta futura.

\subsection{Conclusão}

Neste capítulo, desenvolvemos os métodos de pontos interiores primal-dual para o problema de minimização das perdas de transmissão e custo de geração do pré-despacho DC de um sistema de potência hidroelétrico com base no princípio do mínimo esforço. Escrevemos o problema dual associado ao problema primal do pré-despacho, as condições de otimalidade e aplicamos o método de Newton à estas condições. Resolvemos o sistema resultante da aplicação do método de pontos interiores primal-dual e fizemos um estudo da estrutura matricial, apresentando algumas formas de eliminação de variáveis visando minimizar o esforço computacional. Tal estudo possibilitou reduzir a dimensão da matriz do sistema resultante à ordem do número de geradores. Apresentamos, ainda, os métodos PDPME e PCPME que serviram de base para a implementação, respectivamente, dos métodos de pontos interiores para os problemas primal-dual e preditor-corretor, associados ao problema de fluxo de potência ótimo DC via princípio do mínimo esforço. Finalmente, propomos um método de ponto interior primal-dual utizando uma heurística, relacionada a um parâmetro de perturbação (VPMPD), que se mostrou eficiente na prática, reduzindo significativamente o número de iterações. 


\section{Capítulo 6}

\section{Experimentos Numéricos}

\subsection{Introdução}

Neste capítulo, apresentamos os resultados obtidos pela aplicação dos métodos de pontos interiores primal-dual (PDPME, VPMPD) e preditor-corretor (PCPME), ao problema do pré-despacho de um sistema hidroelétrico usando o princípio do mínimo esforço. Os resultados são comparados, em termos de tempo computacional, quantidade de operações de ponto flutuante e número de iterações, com os obtidos por meio de uma implementação para o modelo de fluxos em redes desenvolvida por (OLIVEIRA; NEPOMUCENO; SOARES, 2005), denominada PDFPO, para o método primal-dual e PCFPO para o método preditorcorretor.

As implementações, tanto para o problema de fluxo em redes, quanto para o modelo que inclui o princípio do mínimo esforço, foram feitas no software Matlab 5.3, sendo que a execução dos programas ocorreu em um Pentium 4 com 2.8 Mhz e 512 Kb de memória RAM.

Foram considerados como testes os sistemas IEEE30 e IEEE118 representando o meio oeste americano, SSE1654 e SSE1732 representando o sul-sudeste centro oeste brasileiro e um equivalente ao sistema interligado nacional (Brasil) com 1993 barras.

O carregamento para cada tempo é dada pelo carregamento básico do sistema sob consideração multiplicado por um fator peso, definido conforme mostra a Tabela 6.1.

A Figura 6.1 mostrada na sequência expressa graficamente a relação entre o intervalo de tempo de 24 horas e o fator peso correspondente a cada hora. 
Tabela 6.1: Fatores de Carga

\begin{tabular}{|c|c|c|c|c|c|}
\hline Hora & Fator & Hora & Fator & Hora & Fator \\
\hline $1: 00$ & 0.7948 & $2: 00$ & 0.7425 & $3: 00$ & 0.7255 \\
$4: 00$ & 0.7222 & $5: 00$ & 0.7345 & $6: 00$ & 0.7816 \\
$7: 00$ & 0.9012 & $8: 00$ & 0.9832 & $9: 00$ & 1.0535 \\
$10: 00$ & 1.0896 & $11: 00$ & 1.0976 & $12: 00$ & 1.0888 \\
$13: 00$ & 1.0557 & $14: 00$ & 1.0823 & $15: 00$ & 1.0814 \\
$16: 00$ & 1.0846 & $17: 00$ & 1.1134 & $18: 00$ & 1.1714 \\
$19: 00$ & 1.2998 & $20: 00$ & 1.2393 & $21: 00$ & 1.1658 \\
$22: 00$ & 1.1089 & $23: 00$ & 1.0000 & $24: 00$ & 0.8828 \\
\hline
\end{tabular}

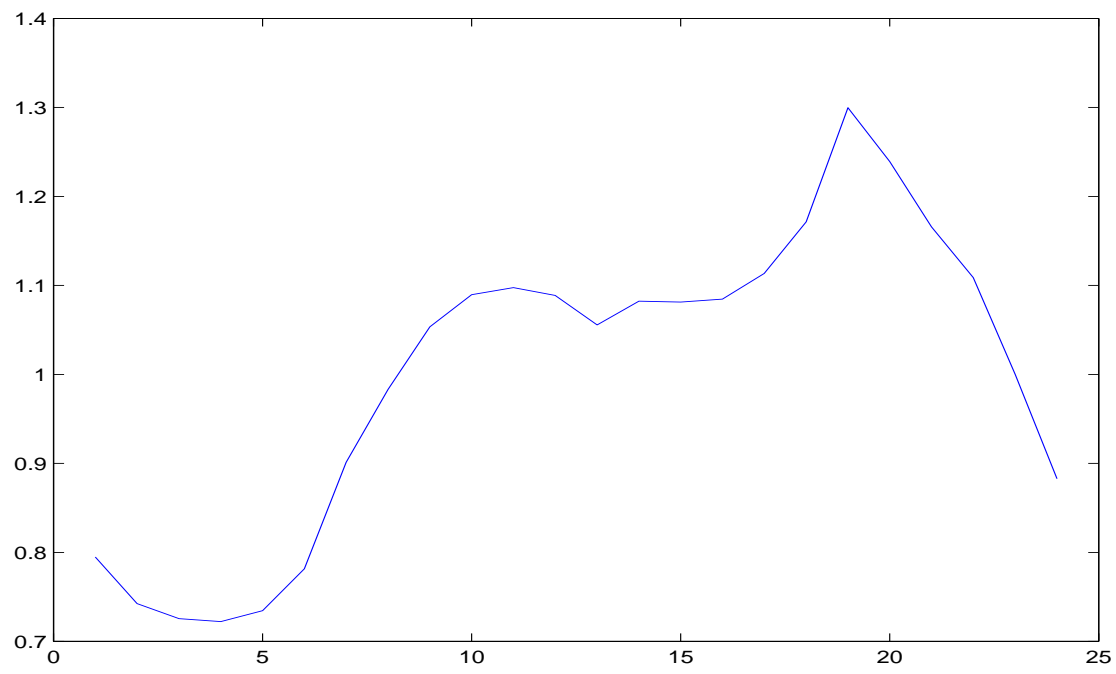

Figura 6.1: Tempo versus Fator peso

Foram adotados os valores $c_{p}=0$ e $c_{\theta}=0$ (funções quadráticas puras) e os custos de geração são os mesmos para todos os geradores. Os limites de geração foram escolhidos de tal forma que não existam restrições de capacidade ativa e as ponderações foram definidas como $\alpha=\beta=1$. Os resultados são apresentados para diferentes intervalos de tempo: $\mathrm{t}=24$ horas, $\mathrm{t}=48$ horas e $\mathrm{t}=72$ horas.

Também, dois tipos de eliminação de variáveis foram considerados resultando em implementações distintas. Para cada eliminação de variáveis consideramos duas formas 
diferentes de calcular as inversas das matrizes que surgem do processo de eliminação de variáveis: uma, utilizando os comandos internos do Matlab e, outra, pelo método de Shermann-Morrisson-Woodbury (DUFF; ERISMAN; REID, 1986).

\subsection{Dados de Entrada}

Os dados definidos a seguir foram utilizados em todas as implementações relacionadas com o PME. O parâmetro $\eta$ que aparece multiplicando cada ponto inicial infactível tem a finalidade de buscar uma convergência em um número menor de iterações. Os valores $\eta=4$ para o primal-dual e $\eta=0.5$ para o preditor-corretor foram satisfatórios no sentido de atender esta condição.

- Dimensão das variáveis primais e duais: $n p=2 t(n+g)$, em que $t=24,48$ ou 72 horas, $n=$ número de linhas e $g=$ número de geradores.

- $\tau=0.9995$

- Parâmetro de centro: $\sigma_{0}=\frac{1}{n p \sqrt{n p}}$

- Critério de parada: $\epsilon=10^{-5}$

- Ponto inicial infactível: Método Primal-Dual

$\eta=4$

Para $i=1,2, \cdots, t$ 


$$
\begin{aligned}
\tilde{p}^{0}(:, i) & =\eta p^{\max } \\
s_{\tilde{p}}^{0}(:, i) & =\tilde{p}^{0}(:, i) \\
w_{\tilde{p}}^{0}(:, i) & =\eta e \\
z_{\tilde{p}}^{0}(:, i) & =w_{\tilde{p}}^{0}(:, i) \\
y_{\tilde{p}}^{0}(:, i) & =0 \\
\theta^{0}(:, i) & =0 \\
s_{\theta_{1}}^{0}(:, i) & =\eta f^{\max } \\
s_{\theta_{2}}^{0}(:, i) & =s_{\theta_{1}}^{0}(:, i) \\
w_{\theta_{1}}^{0}(:, i) & =\eta e^{0} \\
w_{\theta_{2}}^{0}(:, i) & =w_{\theta_{1}}^{0}(:, i)
\end{aligned}
$$

\section{FIM}

\section{Método Preditor-Corretor}

$\eta=0.5$

Para $i=1,2, \cdots, t$

$$
\begin{aligned}
z_{\tilde{p}}^{0}(:, i) & =\eta e \\
w_{\tilde{p}}^{0}(:, i) & =\eta\left(e+z_{\tilde{p}}^{0}(:, i)\right) \\
s_{\tilde{p}}^{0}(:, i) & =\eta\left(p^{\max }-Q^{-1}\left(w_{\tilde{p}}^{0}(:, i)-z_{\tilde{p}}^{0}(:, i)\right)\right) \\
\tilde{p}^{0}(:, i) & =\eta\left(p^{\max }-s_{\tilde{p}}^{0}(:, i)\right) \\
y_{\theta_{\tilde{p}}}^{0}(:, i) & =0 \\
\theta^{0}(:, i) & =0 \\
s_{\theta_{1}}^{0}(:, i) & =\eta f^{\max } \\
s_{\theta_{2}}^{0}(:, i) & =s_{\theta_{1}}^{0}(:, i) \\
w_{\theta_{1}}^{0}(:, i) & =\eta e^{0} \\
w_{\theta_{2}}^{0}(:, i) & =w_{\theta_{1}}^{0}(:, i) \\
F I M &
\end{aligned}
$$


- Critério de convergência Para cada iteração $k$ e em cada $i=1,2, \cdots$, $t$, o critério de convergência é calculado como:

$$
\left[\begin{array}{c}
\frac{\left\|r_{i}\right\|}{1+\left\|\tilde{l}_{i}^{k}\right\|} \\
\frac{\left\|r_{p}\right\|}{1+\left\|p^{\text {max }}\right\|} \\
\frac{\left\|r_{g}\right\|}{1+\left\|c_{p}\right\|} \\
\frac{\left\|r_{t 1}\right\|}{1+\left\|f^{\text {max }}\right\|} \\
\frac{\left\|r_{t 2}\right\|}{1+\left\|f^{m i n}\right\|} \\
\left\|r_{y}\right\|
\end{array}\right] \leq \epsilon
$$

\subsection{Resultados Obtidos}

\subsubsection{Eliminação de Variáveis 1}

Como desenvolvido no capítulo 5, neste processo de redução do sistema (5.47) a (5.57), obtivemos o sistema final:

$$
\sum_{k=1}^{t}\left[Z D Z^{\prime}+D_{\tilde{p}}^{k}\right]^{-1} d y_{a}=r_{m}+\sum_{k=1}^{t}\left[Z D Z^{\prime}+D_{\tilde{p}}^{k}\right]^{-1} r
$$

A matriz $M=Z D Z^{\prime}+D_{\tilde{p}}^{k}$ tem dimensão correspondente ao número de geradores do sistema sob consideração. Ela é simétrica, definida positiva e utilizamos o comando interno $\operatorname{Chol}(M)$ do Matlab para calcular sua inversa em cada tempo. Estes comando faz a decomposição da matriz pelo método de Cholesky. Neste caso, a decomposição de Cholesky foi eficiente e produziu uma ligeira redução no tempo computacional e no número de operações em ponto flutuante, do que se tivéssemos utilizado o operador matricial de divisão à esquerda \, cuja notação é $M \backslash e y e(g)$, em que eye $(g)$ representa a matriz identidade de ordem $g$. Os resultados da implementação com esta forma de calcular a inversa da matriz M, aparecem nas colunas das Tabelas 6.2 a 6.19, com a denominação E1, E1-VPMPD para o primal-dual e E1 para o preditor corretor. Uma alternativa descrita no capítulo 5 para esta forma de eliminação de variáveis é utilizar a fórmula de ShermannMorrisson-Woodbury. Aplicamos esta fórmula uma primeira vez à matriz M, resultando em: 


$$
M^{-1}=\left(D_{\tilde{p}}^{k}\right)^{-1}-\left(D_{\tilde{p}}^{k}\right)^{-1} Z H^{-1} Z^{\prime}\left(D_{\tilde{p}}^{k}\right)^{-1}
$$

em que $H=(D)^{-1}+Z^{\prime}\left(D_{\tilde{p}}^{k}\right)^{-1} Z$.

Os resultados da implementação com esta forma de calcular a inversa, aparecem nas colunas das tabelas 6.2 a 6.19, com denominação E1-SMW tanto para o método primal-dual quanto para o método preditor-corretor.

\subsubsection{Eliminação de Variáveis 2}

No processo de redução do sistema (5.47) a (5.57), obtivemos o sistema final:

$$
\sum_{k=1}^{t}\left[\left(D_{\tilde{p}}^{k}\right)^{-1}-\left(D_{\tilde{p}}^{k}\right)^{-1} E^{\prime} M^{-1} E\left(D_{\tilde{p}}^{k}\right)^{-1}\right] d y_{a}=r_{m}+\sum_{k=1}^{t}\left(D_{\tilde{p}}^{k}\right)^{-1}\left[r_{b}+E^{\prime} M^{1} r\right]
$$

em que

$$
M=B\left(\hat{B}^{k}\right)^{-1} B^{\prime}+E\left(D_{\tilde{p}}^{k}\right)^{-1} E^{\prime}
$$

A matriz $M$ tem dimensão correspondente ao número de barras do sistema sob consideração e é simétrica, definida positiva e não singular.

A matriz $\hat{B}^{k}$ que aparece na formação de $M$ também é simétrica, definida positiva e não singular.

Observamos que, neste caso, utilizando o operador matricial de divisão à esquerda

$\backslash$, para calcular a inversa destas matrizes, os resultados em termos de tempo computacional e número de operações de ponto flutuante, eram melhores do que se tivéssemos utilizado a decomposição de Cholesky. Fizemos, também, neste tipo de redução do sistema, uma implementação considerando o cálculo da inversa da matriz $M$, pela fórmula de Shermann-Morrisson-Woodbury. Para este caso, o método primal-dual não convergiu para o sistema de 1654 barras. A partir da décima primeira iteração o erro começou a crescer de tal forma em iterações posteriores tornou-se estacionário. Não temos uma explicação plausível para este fato. Outra ocorrência inusitada é que para o sistema de 1993 barras (Brasil) a convergência do primal-dual ocorreu em um tempo computacional surpreendentemente menor do que o esperado. Tomando por base os resultados de E2 
para o período de 48 horas, deixamos de apresentar os resultados de E2 para o período de 72 horas, bem como os resultados de E2-SMW para os períodos de 48 e 72 horas, por supôr que o tempo computacional para estes casos excede 24 horas.

Os resultados são apresentados nas colunas das Tabelas 6.2 a 6.19, denotadas por E2 e E2-SMW, tanto para o método primal-dual quanto para o método preditor-corretor.

\subsubsection{Análise dos Resultados}

Observando os resultados mostrados nas Tabelas 6.3, 6.5, 6.9, 6.11, 6.15 e 6.17, verificamos que:

- A forma de eliminação de variáveis E1-SMW foi superior a todas as outras formas de implementação que utilizam o princípio do mínimo esforço, para todos os sistemas.

- A implementação para o modelo de fluxo em redes (PCFPO) foi superior à forma de Eliminação de Variáveis E1-SMW em todos os sistemas.

Assim, considerando o tempo computacional e o número de operações em ponto flutuante (Flops), o Método Preditor-Corretor implementado via fluxo em redes é superior a qualquer uma das implementações via princípio do Mínimo Esforço, qualquer que seja o sistema utilizado.

De maneira análoga, os resultados mostrados nas Tabelas 6.2, 6.4, 6.8, 6.10, 6.14 e 6.16, demonstram que, exceto para o sistema de 30 barras, a forma de eliminação de variáveis E1-SMW foi superior a todas as outras formas de implementação utilizando o princípio do mínimo esforço. Novamente, a implementação para o modelo de fluxo em redes $(\mathrm{PDFPO})$ foi superior à forma de Eliminação de Variáveis E1-SMW para todos os sistemas.

Assim, considerando o tempo computacional e o número de operações em ponto flutuante (Flops), o Método Primal Dual implementado via fluxo em redes é superior a qualquer uma das implementações via Princípio do Mínimo Esforço, qualquer que seja o sistema utilizado.

Com relação ao número de iterações verificamos que: 
- Para o Método Preditor Corretor as implementações utilizando o Princípio do Mínimo Esforço convergiram para o mesmo número de iterações, para cada sistema teste.

Estas implementações convergiram em menos iterações nos períodos de 48 horas e 72 horas, qualquer que fosse o sistema teste, do que a implementação via fluxo em redes (PCFPO). Para o período de 24 horas, as implementações via Princípio do Mínimo Esforço convergiram, na pior das hipóteses, com o mesmo número de iterações que a implementação utilizando fluxo em redes. Estes resultados podem ser vistos nas Tabelas 6.7, 6.13 e 6.19 .

- Para o Método Primal Dual as implementações utilizando o princípio do mínimo esforço foram superiores à implementação via fluxo em redes (PDFPO) para todos os sistemas testes e períodos de tempo. Entretanto, é importante ressaltar que o Método VPMPD proposto (Seção 5.9) foi o que obteve melhor desempenho entre todos, principalmente para os sistemas maiores ou iguais a 1654 barras. Estes resultados podem ser vistos nas Tabelas 6.6, 6.12 e 6.18. 
Tabela 6.2: Método Primal-Dual - Tempo Computacional - 24h

\begin{tabular}{|c|c|c|c|c|c|c|}
\hline SISTEMAS & PDFPO & E1 & E1-SMW & E1-VPMPD & E2 & E2-SMW \\
\hline IEEE30 & 0.2820 & 0.0310 & 0.0460 & 0.0790 & 0.1250 & 0.0790 \\
\hline IEEE118 & 3.9380 & 4.0000 & 3.2030 & 2.5750 & 10.7340 & 7.0780 \\
\hline SSE1654 & 301.078 & 1343.468 & 844.375 & 685.672 & 16152.422 & - \\
\hline SSE1732 & 316.234 & 1440.775 & 879.703 & 508.906 & 18354.469 & 20838.671 \\
\hline BRASIL1993 & 410.640 & 2276.359 & 1616.641 & 838.406 & 28448.360 & 1488.578 \\
\hline
\end{tabular}

Tabela 6.3: Método Preditor-Corretor- Tempo Computacional - 24h

\begin{tabular}{|c|c|c|c|c|c|}
\hline SISTEMAS & PCFPO & E1 & E1-SMW & E2 & E2-SMW \\
\hline IEEE30 & 0.2500 & 0.0620 & 0.0470 & 0.2030 & 0.1250 \\
\hline IEEE118 & 2.6710 & 4.5000 & 2.8430 & 9.9070 & 6.4840 \\
\hline SSE1654 & 213.453 & 1838.203 & 1019.703 & 27748.891 & 15066.641 \\
\hline SSE1732 & 224.032 & 1470.891 & 1075.359 & 28162.485 & 16175.016 \\
\hline BRASIL1993 & 272.813 & 2782.156 & 1702.875 & 47599.625 & 1759.687 \\
\hline
\end{tabular}


Tabela 6.4: Método Primal-Dual - Flops - 24h

\begin{tabular}{|c|c|c|c|c|c|c|}
\hline SISTEMAS & PDFPO & E1 & E1-SMW & E1-VPMPD & E2 & E2-SMW \\
\hline IEEE30 & 1525090 & 886512 & 646512 & 908637 & 4189212 & 3614148 \\
\hline IEEE118 & 25660470 & 54279363 & 41600764 & 32575754 & 95234631 & 104914409 \\
\hline SSE1654 & $1.348 \mathrm{e}+10$ & $2.975 \mathrm{e}+11$ & $1.591 \mathrm{e}+11$ & $1.352 \mathrm{e}+11$ & $2.713 \mathrm{e}+12$ & - \\
\hline SSE1732 & $1.203 \mathrm{e}+10$ & $2.950 \mathrm{e}+11$ & $1.567 \mathrm{e}+11$ & $1.072 \mathrm{e}+11$ & $3.106 \mathrm{e}+12$ & $5.451 \mathrm{e}+12$ \\
\hline BRASIL1993 & $2.258 \mathrm{e}+10$ & $5.172 \mathrm{e}+11$ & $2.762 \mathrm{e}+11$ & $1.880 \mathrm{e}+11$ & $4.772 \mathrm{e}+12$ & $2.759 \mathrm{e}+11$ \\
\hline
\end{tabular}

Tabela 6.5: Método Preditor-Corretor - Flops - 24h

\begin{tabular}{|c|c|c|c|c|c|}
\hline SISTEMAS & PCFPO & E1 & E1-SMW & E2 & E2-SMW \\
\hline IEEE30 & 1505018 & 1732526 & 1198238 & 6553466 & 4627826 \\
\hline IEEE118 & 151725123 & 558990928 & 363375624 & 785847419 & 675385783 \\
\hline SSE1654 & $8.157 \mathrm{e}+09$ & $3.697 \mathrm{e}+11$ & $1.921 \mathrm{e}+11$ & $4.321 \mathrm{e}+12$ & $3.051 \mathrm{e}+12$ \\
\hline SSE1732 & $7.317 \mathrm{e}+09$ & $3.150 \mathrm{e}+11$ & $1.633 \mathrm{e}+11$ & $4.349 \mathrm{e}+12$ & $2.992 \mathrm{e}+12$ \\
\hline BRASIL1993 & $1.338 \mathrm{e}+10$ & $6.425 \mathrm{e}+11$ & $3.335 \mathrm{e}+11$ & $7.905 \mathrm{e}+12$ & $3.333 \mathrm{e}+11$ \\
\hline
\end{tabular}


Tabela 6.6: Método Primal-Dual - Iterações - 24h

\begin{tabular}{|c|c|c|c|c|c|c|}
\hline SISTEMAS & PDFPO & E1 & E1-SMW & E1-VPMPD & E2 & E2-SMW \\
\hline IEEE30 & 5 & 1 & 1 & 1 & 1 & 1 \\
\hline IEEE118 & 7 & 5 & 5 & 3 & 5 & 5 \\
\hline SSE1654 & 12 & 11 & 11 & 5 & 11 & - \\
\hline SSE1732 & 12 & 11 & 11 & 4 & 11 & 11 \\
\hline BRASIL1993 & 12 & 11 & 11 & 4 & 11 & 11 \\
\hline
\end{tabular}

Tabela 6.7: Método Preditor-Corretor - Iterações - 24h

\begin{tabular}{|c|c|c|c|c|c|}
\hline SISTEMAS & PCFPO & E1 & E1-SMW & E2 & E2-SMW \\
\hline IEEE30 & 3 & 1 & 1 & 1 & 1 \\
\hline IEEE118 & 4 & 3 & 3 & 3 & 3 \\
\hline SSE1654 & 7 & 7 & 7 & 7 & 7 \\
\hline SSE1732 & 7 & 6 & 6 & 6 & 6 \\
\hline BRASIL1993 & 7 & 7 & 7 & 7 & 7 \\
\hline
\end{tabular}


Tabela 6.8: Método Primal-Dual - Tempo Computacional - 48h

\begin{tabular}{|c|c|c|c|c|c|c|}
\hline SISTEMAS & PDFPO & E1 & E1-SMW & E1-VPMPD & E2 & E2-SMW \\
\hline IEEE30 & 0.5470 & 0.0630 & 0.0940 & 0.0630 & 0.2650 & 0.1410 \\
\hline IEEE118 & 7.6880 & 7.7660 & 6.3590 & 4.8600 & 21.9070 & 14.2970 \\
\hline SSE1654 & 605.6410 & 2696.3910 & 1693.4840 & 1372.6880 & 32348.3120 & $>24 \mathrm{~h}$ \\
\hline SSE1732 & 633.157 & 2802.000 & 1753.484 & 1017.219 & 37241.109 & $>24 \mathrm{~h}$ \\
\hline BRASIL1993 & 822.594 & 4578.172 & 2869.031 & 1688.219 & 61998.344 & $>24 \mathrm{~h}$ \\
\hline
\end{tabular}

Tabela 6.9: Método Preditor-Corretor - Tempo Computacional - 48h

\begin{tabular}{|c|c|c|c|c|c|}
\hline SISTEMAS & PCFPO & E1 & E1-SMW & E2 & E2-SMW \\
\hline IEEE30 & 0.4840 & 0.1250 & 0.0940 & 0.3750 & 0.2340 \\
\hline IEEE118 & 5.2810 & 8.4380 & 5.5630 & 19.7810 & 13.2340 \\
\hline SSE1654 & 427.9220 & 3157.1090 & 1712.8440 & $>24 \mathrm{~h}$ & $>24 \mathrm{~h}$ \\
\hline SSE1732 & 448.250 & 2931.859 & 1785.328 & $>24 \mathrm{~h}$ & $>24 \mathrm{~h}$ \\
\hline BRASIL1993 & 544.265 & 4010.016 & 2431.844 & $>24 \mathrm{~h}$ & $>24 \mathrm{~h}$ \\
\hline
\end{tabular}


Tabela 6.10: Método Primal-Dual - Flops - 48h

\begin{tabular}{|c|c|c|c|c|c|c|}
\hline SISTEMAS & PDFPO & E1 & E1-SMW & E1-VPMPD & E2 & E2-SMW \\
\hline IEEE30 & 3047324 & 1772736 & 2617262 & 1816989 & 8378028 & 7227900 \\
\hline IEEE118 & 51249754 & 108508019 & 83150819 & 65121083 & 190019437 & 209774415 \\
\hline SSE1654 & $2.66 \mathrm{e}+10$ & $5.95 \mathrm{e}+11$ & $3.18 \mathrm{e}+11$ & $2.71 \mathrm{e}+11$ & $5.43 \mathrm{e}+12$ & - \\
\hline SSE1732 & $2.40 \mathrm{e}+10$ & $5.90 \mathrm{e}+11$ & $3.13 \mathrm{e}+11$ & $2.14 \mathrm{e}+11$ & $6.21 \mathrm{e}+12$ & - \\
\hline BRASIL1993 & $4.51 \mathrm{e}+10$ & $1.03 \mathrm{e}+12$ & $5.52 \mathrm{e}+11$ & $3.76 \mathrm{e}+11$ & $9.54 \mathrm{e}+12$ & - \\
\hline
\end{tabular}

Tabela 6.11: Método Preditor-Corretor - Flops - 48h

\begin{tabular}{|c|c|c|c|c|c|}
\hline SISTEMAS & PCFPO & E1 & E1-SMW & E2 & E2-SMW \\
\hline IEEE30 & 3383662 & 3685838 & 2617262 & 13327610 & 9476330 \\
\hline IEEE118 & 30550209 & 112087993 & 72965044 & 157417430 & 135379160 \\
\hline SSE1654 & $1.633 \mathrm{e}+10$ & $6.338 \mathrm{e}+11$ & $3.295 \mathrm{e}+11$ & - & - \\
\hline SSE1732 & $1.465 \mathrm{e}+10$ & $6.301 \mathrm{e}+11$ & $3.267 \mathrm{e}+11$ & - & - \\
\hline BRASIL1993 & $2.677 \mathrm{e}+10$ & $9.179 \mathrm{e}+11$ & $4.765 \mathrm{e}+11$ & - & - \\
\hline
\end{tabular}


Tabela 6.12: Método Primal-Dual - Iterações - 48h

\begin{tabular}{|c|c|c|c|c|c|c|}
\hline SISTEMAS & PDFPO & E1 & E1-SMW & E1-VPMPD & E2 & E2-SMW \\
\hline IEEE30 & 5 & 1 & 1 & 1 & 1 & 1 \\
\hline IEEE118 & 7 & 5 & 5 & 3 & 5 & 5 \\
\hline SSE1654 & 12 & 11 & 11 & 5 & 11 & - \\
\hline SSE1732 & 12 & 11 & 11 & 4 & 11 & - \\
\hline BRASIL1993 & 12 & 11 & 11 & 4 & 11 & - \\
\hline
\end{tabular}

Tabela 6.13: Método Preditor-Corretor - Iterações - 48h

\begin{tabular}{|c|c|c|c|c|c|}
\hline SISTEMAS & PCFPO & E1 & E1-SMW & E2 & E2-SMW \\
\hline IEEE30 & 3 & 1 & 1 & 1 & 1 \\
\hline IEEE118 & 4 & 3 & 3 & 3 & 3 \\
\hline SSE1654 & 7 & 6 & 6 & - & - \\
\hline SSE1732 & 7 & 6 & 6 & - & - \\
\hline BRASIL1993 & 7 & 5 & 5 & - & - \\
\hline
\end{tabular}


Tabela 6.14: Método Primal-Dual - Tempo Computacional - 72h

\begin{tabular}{|c|c|c|c|c|c|c|}
\hline SISTEMAS & PDFPO & E1 & E1-SMW & E1-VPMPD & E2 & E2-SMW \\
\hline IEEE30 & 0.7500 & 0.1090 & 0.0780 & 0.1090 & 0.3750 & 0.2030 \\
\hline IEEE118 & 11.7190 & 11.5780 & 9.6560 & 7.1400 & 32.2350 & 21.5160 \\
\hline SSE1654 & 908.297 & 4074.094 & 2541.953 & 2066.641 & $>24 \mathrm{~h}$ & $>24 \mathrm{~h}$ \\
\hline SSE1732 & 951.687 & 4202.500 & 2640.813 & 1526.078 & $>24 \mathrm{~h}$ & $>24 \mathrm{~h}$ \\
\hline BRASIL1993 & 1233.812 & 6954.859 & 4310.593 & 2490.859 & $>24 \mathrm{~h}$ & $>24 \mathrm{~h}$ \\
\hline
\end{tabular}

Tabela 6.15: Método Preditor-Corretor - Tempo Computacional - 72h

\begin{tabular}{|c|c|c|c|c|c|}
\hline SISTEMAS & PCFPO & E1 & E1-SMW & E2 & E2-SMW \\
\hline IEEE30 & 0.7030 & 0.1880 & 0.1410 & 0.5630 & 0.3600 \\
\hline IEEE118 & 7.9220 & 12.1090 & 8.4060 & 29.9060 & 26.2030 \\
\hline SSE1654 & 642.750 & 4711.641 & 2622.281 & $>24 \mathrm{~h}$ & $>24 \mathrm{~h}$ \\
\hline SSE1732 & 676.328 & 4590.063 & 2686.890 & $>24 \mathrm{~h}$ & $>24 \mathrm{~h}$ \\
\hline BRASIL1993 & 818.453 & 6006.266 & 4144.328 & $>24 \mathrm{~h}$ & $>24 \mathrm{~h}$ \\
\hline
\end{tabular}


Tabela 6.16: Método Primal-Dual - Flops - 72h

\begin{tabular}{|c|c|c|c|c|c|c|}
\hline SISTEMAS & PDFPO & E1 & E1-SMW & E1-VPMPD & E2 & E2-SMW \\
\hline IEEE30 & 4568004 & 2658960 & 1938960 & 2725341 & 12566844 & 10841652 \\
\hline IEEE118 & $7.68 \mathrm{e}+08$ & $1.63 \mathrm{e}+09$ & $1.25 \mathrm{e}+09$ & $9.77 \mathrm{e}+08$ & $2.86 \mathrm{e}+09$ & $3.15 \mathrm{e}+09$ \\
\hline SSE1654 & $4.04 \mathrm{e}+10$ & $8.93 \mathrm{e}+11$ & $4.77 \mathrm{e}+11$ & $4.06 \mathrm{e}+11$ & - & - \\
\hline SSE1732 & $3.61 \mathrm{e}+10$ & $8.85 \mathrm{e}+11$ & $4.70 \mathrm{e}+11$ & $3.22 \mathrm{e}+11$ & - & - \\
\hline BRASIL1993 & $6.76 \mathrm{e}+10$ & $1.55 \mathrm{e}+12$ & $8.29 \mathrm{e}+12$ & $5.64 \mathrm{e}+11$ & - & - \\
\hline
\end{tabular}

Tabela 6.17: Método Preditor-Corretor - Flops - 72h

\begin{tabular}{|c|c|c|c|c|c|}
\hline SISTEMAS & PCFPO & E1 & E1-SMW & E2 & E2-SMW \\
\hline IEEE30 & 5640898 & 5860334 & 4257470 & 20322938 & 14546018 \\
\hline IEEE118 & 461202316 & 1685991210 & 1099146264 & $2.3610 \mathrm{e}+09$ & $2.2749 \mathrm{e}+09$ \\
\hline SSE1654 & $2.454 \mathrm{e}+10$ & $9.509 \mathrm{e}+11$ & $4.944 \mathrm{e}+11$ & - & - \\
\hline SSE1732 & $2.202 \mathrm{e}+10$ & $9.453 \mathrm{e}+11$ & $4.902 \mathrm{e}+11$ & - & - \\
\hline BRASIL1993 & $4.021 \mathrm{e}+10$ & $1.377 \mathrm{e}+12$ & $7.148 \mathrm{e}+11$ & - & - \\
\hline
\end{tabular}


Tabela 6.18: Método Primal-Dual - Iterações - 72h

\begin{tabular}{|c|c|c|c|c|c|c|}
\hline SISTEMAS & PDFPO & E1 & E1-SMW & E1-VPMPD & E2 & E2-SMW \\
\hline IEEE30 & 5 & 1 & 1 & 1 & 1 & 1 \\
\hline IEEE118 & 7 & 5 & 5 & 3 & 5 & 5 \\
\hline SSE1654 & 12 & 11 & 11 & 5 & - & - \\
\hline SSE1732 & 12 & 11 & 11 & 4 & - & - \\
\hline BRASIL1993 & 12 & 11 & 11 & 4 & - & - \\
\hline
\end{tabular}

Tabela 6.19: Método Preditor-Corretor - Iterações - 72h

\begin{tabular}{|c|c|c|c|c|c|}
\hline SISTEMAS & PCFPO & E1 & E1-SMW & E2 & E2-SMW \\
\hline IEEE30 & 3 & 1 & 1 & 1 & 1 \\
\hline IEEE118 & 4 & 3 & 3 & 3 & 3 \\
\hline SSE1654 & 7 & 6 & 6 & - & - \\
\hline SSE1732 & 7 & 6 & 6 & - & - \\
\hline BRASIL1993 & 7 & 5 & 5 & - & - \\
\hline
\end{tabular}




\subsection{Conclusão}

Neste capítulo, apresentamos os resultados das implementações dos métodos de pontos interiores primal-dual e preditor-corretor, utilizando o princípio do mínimo esforço. Comparamos, inicialmente, estes métodos entre si, em termos de tempo computacional, número de operações em ponto flutuante e número de iterações. Observamos que a redução do sistema original pela eliminação de variáveis 1 (ver capítulo 5) foi mais eficiente que a redução do sistema pela eliminação de variáveis 2. De fato, no primeiro caso, a matriz a ser invertida tem dimensão do número de geradores $g$, enquanto que no segundo caso, existem duas matrizes para inverter, ambas com dimensão do número de barras $m$, em que $g<<m$. Verificamos, ainda, que a utilização da fórmula de Shermann-Morrisson-Woodbury produziu uma implementação mais eficiente. Entretanto, a implementação que utilizou a eliminação de variáveis 1 e considerou um parâmetro de perturbação (E1-VPMPD) foi a mais eficiente entre todas relacionadas com o método primal-dual, principalmente no aspecto número de iterações. Neste aspecto, ela foi superior à implementação do fluxo de potência ótimo (PDFPO).

No caso do método preditor-corretor, a implementação utilizando o Princípio do Mínimo Esforço (PCPME), que considerou o cálculo da inversa pela fórmula de ShermannMorrisson-Woodbury (SMW) (E1-SMW), também foi superior à qualquer outra implementação do Princípio do Mínimo Esforço. Quando comparada à implementação do fluxo de potência ótimo (PCFPO), esta foi superior, exceto no que tange ao número de iterações. 


\section{Capítulo 7}

\section{Conclusões e Perspectivas Futuras}

Neste trabalho, formulamos o modelo matemático do problema do pré-despacho de sistemas hidroelétricos e fizemos uma simplificação do mesmo utilizando o princípio do mínimo esforço.

Objetivando a implementação do modelo simplificado, fizemos um estudo da estrutura matricial resultante da aplicação dos métodos de pontos interiores primal-dual e preditor-corretor. Verificamos que das duas formas de eliminação de variáveis apresentadas, a primeira (E1) foi a que apresentou melhores resultados do ponto de vista do esforço computacional.

Apresentamos uma análise geral sobre otimização e métodos de pontos interiores, com ênfase nos métodos primal-dual e preditor corretor pelo que representam na literatura em termos teóricos e práticos, além do fato de que os mesmos seriam utilizados na implementação do modelo simplificado.

Apresentamos uma proposta de um método de pontos interiores primal-dual cuja novidade consiste na definição de uma heurística para a escolha de um parâmetro de perturbação. Este método mostrou-se robusto e eficiente, principalmente com relação ao número de iterações.

Finalmente, fizemos implementações computacionais dos métodos descritos, levando em consideração o estudo da estrutura matricial e as eliminações de variáveis propostas. Comparações com o modelo de fluxo em redes mostraram que implementar o modelo simplificado pode ser, no mínimo, tão vantajoso quanto a implementação existente no modelo de fluxos em rede. 
- A implementação do método VPMPD seja aperfeiçoada com relação ao cálculo das inversas das matrizes finais. Por exemplo, a fórmula de Sherman-MorrisonWoodbury não foi testada para este caso.

- O método VPMPD seja implementado para o método preditor-corretor.

- O método VPMPD seja implementado para o modelo de fluxo em redes (PDFPO e PCFPO).

- Seja desenvolvida uma análise teórica das propriedades de convergência do método VPMPD, bem como de sua complexidade computacional.

- Seja verificado a razão pela qual a implementação E2-SMW não convergiu para o sistema composto de 1654 barras. 


\section{Trabalhos Publicados}

- Pré-Despacho de Sistemas de Potência Hidroelétricos via Métodos de Pontos Interiores. Carvalho, L.M.R.; Oliveira, A.R.L. XXXV SBPO - Simpósio Brasileiro de Pesquisa Operacional, Novembro, 2003, Natal - RN, Trabalho Completo em CDROM.

- Simplificação de Modelos de Otimização - Aplicação ao Problema de Pré-Despacho de Sistemas de Potência. Carvalho, L.M.R.; Oliveira, A.R.L. XXVI CNMAC Congresso Nacional de Matemática Aplicada e Computacional, Setembro, 2003, São José do Rio Preto - SP, Resumo em CD-ROM.

- O Problema do Pré-Despacho DC via Pontos Interiores e o Princípio do Mínimo Esforço. Carvalho, L.M.R.; Oliveira, A.R.L. CBA 2004 - XV Congresso da Sociedade Brasileira de Automática, Setembro, 2004, Gramado - RS. Trabalho completo em CD-ROM.

- Métodos de Pontos Interiores Aplicados ao Pré-Despacho de um Sistema Hidroelétrico Usando o Princípio do Mínimo Esforço - Comparação com o Modelo de Fluxo em Redes. Carvalho, L.M.R.; Oliveira, A.R.L. IX Simpósio de Teses e Dissertações do ICMC-USP, Novembro, 2004, São Carlos - SP, Resumo em CD-ROM.

- O Problema do Pré-Despacho DC via Método de Pontos Interiores Primal-Dual e o Princípio do Mínimo Esforço. Carvalho, L.M.R.; Oliveira, A.R.L. XXXVI SBPO Simpósio Brasileiro de Pesquisa Operacional, Novembro, 2004, São João Del Rey MG, Resumo em CD-ROM.

- Comparação entre duas Formulações do Problema de Minimização das Perdas no Pré-Despacho por Métodos de Pontos Interiores. Carvalho, L.M.R.; Oliveira, A.R.L. 
V ERMAC - R3 - Encontro Regional de Matemática Aplicada e Computacional, Novembro, 2005, Natal - RN, Trabalho Completo em CD-ROM. 


\section{APÊNDICE A}

\section{O Método de Newton}

O Método de Newton constitui uma ferramente indispensável para o métodos de pontos interiores. De fato, os métodos de pontos interiores podem ser vistos como a aplicação deste método às condições de otimalidade. Assim sendo, vamos definir o método de Newton para funções de uma e várias variáveis.

\section{Funções de uma variável}

Considere o problema de calcular os zeros da função $\phi(x)$, ou seja, resolver o problema $\phi(x)=0$. Expandindo $\phi(x)$ pela série de Taylor em torno de $x=x^{k}, n \geq 0$, temos que:

$$
\phi(x)=\phi\left(x^{k}\right)+\phi^{\prime}\left(x^{k}\right)\left(x-x^{k}\right)+\phi^{\prime \prime}\left(x^{k}\right) \frac{\left(x-x^{k}\right)^{2}}{2 !}+\ldots
$$

em que

$$
\phi^{\prime}\left(x^{k}\right)=\frac{d \phi}{d x}\left(x^{k}\right), \quad \phi^{\prime \prime}\left(x^{k}\right)=\frac{d^{2} \phi}{d x^{2}}\left(x^{k}\right), \ldots
$$

Considerando apenas os dois primeiros termos da série (1), obtemos a seguinte aproximação linear para $\phi(x)$ :

$$
\phi(x) \approx \phi\left(x^{k}\right)+\phi^{\prime}\left(x^{k}\right)\left(x-x^{k}\right)
$$

Para $\phi(x)=0$, a equação (2) pode ser escrita como:

$$
x=x^{k}-\frac{\phi\left(x^{k}\right)}{\phi^{\prime}\left(x^{k}\right) .}
$$


A partir da equação (3), é possível construir um método iterativo para resolver a equação $f(x)=0$, reescrevendo-a na forma:

$$
x^{k+1}=x^{k}-\frac{\phi\left(x^{k}\right)}{\phi^{\prime}\left(x^{k}\right)} .
$$

A equação (4) é conhecida como método de Newton e é o procedimento mais utilizado para encontrar as raízes de uma equação. Ele pode ser generalizado para resolver sistemas não lineares.

A Figura (1) mostra a interpretação geométrica do método de Newton para funções de uma variável.

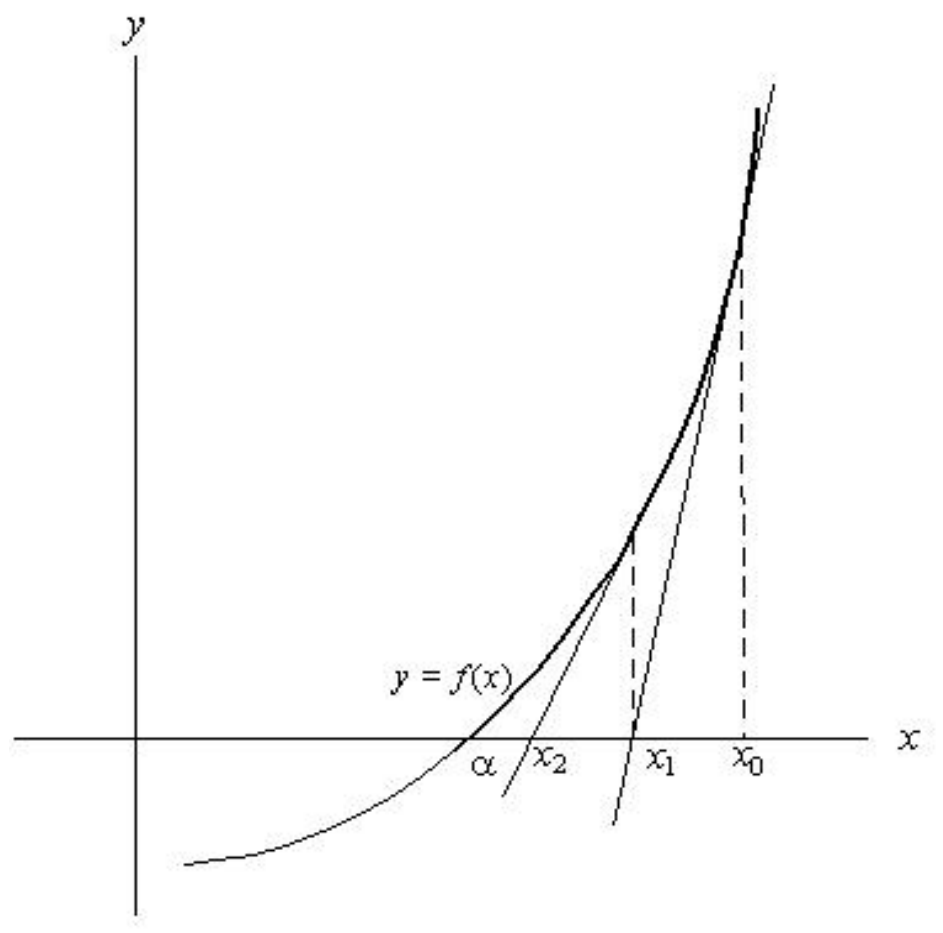

Figura 1: Interpretação geométrica do método de Newton para funções de uma variável

2. Funções de várias variáveis

Seja $F_{i}(x)=0$ um sistema de $n$ equações não lineares e $x$ um vetor de $n$. Aplicando a cada uma destas equações o Teorema de Taylor para funções de várias variáveis e, considerando a aproximação linear, é possível aproximar a solução $F_{i}(x)=0$ em torno do ponto $x=x^{k}$ na forma: 


$$
F_{i}(x) \approx F_{i}\left(x^{k}\right)+\left[\nabla F_{i}\left(x^{k}\right)\right]^{\prime}\left(x-x^{k}\right)
$$

em que

$$
\nabla F_{i}\left(x^{k}\right)=\frac{\partial F_{i}}{\partial x_{1}}\left(x^{k}\right)+\frac{\partial F_{i}}{\partial x_{2}}\left(x^{k}\right)+\ldots+\frac{\partial F_{i}}{\partial x_{n}}\left(x^{0}\right) .
$$

Igualando (5) a zero, podemos escrever o seguinte sistema linear de $n$ equações:

$$
\begin{aligned}
{\left[\nabla F_{1}\left(x^{k}\right)\right]^{\prime}\left(x-x^{k}\right) } & =-F_{1}\left(x^{k}\right) \\
{\left[\nabla F_{2}\left(x^{k}\right)\right]^{\prime}\left(x-x^{k}\right) } & =-F_{2}\left(x^{k}\right) \\
\vdots & \\
{\left[\nabla F_{n}\left(x^{k}\right)\right]^{\prime}\left(x-x^{k}\right) } & =-F_{n}\left(x^{k}\right)
\end{aligned}
$$

Seja

$$
J\left(x^{k}\right)=\left[\begin{array}{r}
\left(\nabla F_{1}\left(x^{k}\right)\right)^{\prime} \\
\vdots \\
\left(\nabla F_{n}\left(x^{k}\right)\right)^{\prime}
\end{array}\right],
$$

o Jacobiano de $F_{i}$ no ponto $x^{k}$. Então, a equação (6) pode ser reescrita como:

$$
J\left(x^{k}\right)\left(x-x^{k}\right)=-F\left(x^{k}\right)
$$

em que

$$
F\left(x^{k}\right)=\left[\begin{array}{r}
F_{1}\left(x^{k}\right) \\
\vdots \\
F_{n}\left(x^{k}\right)
\end{array}\right] .
$$

Multiplicando a equação (7) por $\left[J\left(x^{k}\right)\right]^{-1}$, obtemos

$$
x=x^{k}-\left[J\left(x^{k}\right)\right]^{-1} F\left(x^{k}\right) .
$$


A partir da equação (8) é possível construir um método iterativo para resolver o sistema $F_{i}(x)=k$, reescrevendo-o na forma:

$$
x^{k+1}=x^{k}-\left[J\left(x^{k}\right)\right]^{-1} F\left(x^{k}\right)
$$

que é o método de Newton para sistemas não lineares. 


\section{APÊNDICE B}

Mostraremos, neste apêndice, que uma matriz quadrada, simétrica e singular, com características idênticas à matriz de susceptância definida no capítulo 2, Seção 2.4, pode ser transformada em uma matriz não-singular, eliminando uma linha e uma coluna da mesma.

Teorema .1 Seja A uma matriz quadrada de ordem n, simétrica, singular e satisfazendo a seguinte condição:

$$
a_{i i}=-\sum_{j=1}^{n} a_{i j}, \quad i=1,2, \cdots, n, \quad i \neq j .
$$

Suponha que se retire de A a k-ésima linha e a k-ésima coluna. Então,

1. a matriz $B$ resultante, de ordem $(n-1)$ é não singular $e$,

2. seja $b$ o vetor cujas coordenadas sejam exatamente os elementos da $k$-ésima linha de A, com exceção do elemento de ordem $k$, a seguinte propriedade é verdadeira:

$$
b^{\prime} \cdot B^{-1}=\left[\begin{array}{llll}
-1 & -1 & \ldots & -1
\end{array}\right]
$$

A demonstração da parte (1) deste teorema depende da seguinte definição encontrada em (ATKINSON, 1978; CONTE; BOOR, 1981),

Uma matriz $Y$ de ordem $n$ é diagonalmente dominante, se

$$
\left|y_{i i}\right|>\sum_{j=1}^{n}\left|y_{i j}\right|, \quad i=1,2, \cdots, n, \quad i \neq j,
$$

e do seguinte resultado cuja demonstração pode ser encontrada em (CONTE; BOOR, 1981): 
Se $Y$ é diagonalmente dominante, então, $Y$ é invertível.

De acordo com as hipóteses do Teorema 1 e os resultados obtidos em (ATKINSON, 1978; CONTE; BOOR, 1981), a matriz $B$ é invertível.

Para mostrar a parte (2), consideremos as matrizes $A, B$ e o vetor $b$ como descritos no Teorema 1.

$$
\begin{aligned}
& A=\left[\begin{array}{rrrrrrr}
a_{11} & a_{12} & a_{13} & \ldots & a_{1 k} & \ldots & a_{1 n} \\
a_{12} & a_{22} & a_{23} & \ldots & a_{2 k} & \ldots & a_{2 n} \\
a_{13} & a_{23} & a_{33} & \ldots & a_{3 k} & \ldots & a_{3 n} \\
\vdots & \vdots & \vdots & \ldots & \vdots & \ldots & \vdots \\
a_{1 k} & a_{2 k} & a_{3 k} & \ldots & a_{k k} & \ldots & a_{n k} \\
\vdots & \vdots & \vdots & \ldots & \vdots & \ldots & \vdots \\
a_{1 n} & a_{2 n} & a_{3 n} & \ldots & a_{k n} & \ldots & a_{n n}
\end{array}\right] \\
& B=\left[\begin{array}{rrrrrrrr}
a_{11} & a_{12} & a_{13} & \ldots & a_{1(k-1)} & a_{1(k+1)} & \ldots & a_{1 n} \\
a_{12} & a_{22} & a_{23} & \ldots & a_{2(k-1)} & a_{2(k+1)} & \ldots & a_{2 n} \\
a_{13} & a_{23} & a_{33} & \ldots & a_{3(k-1)} & a_{3(k+1)} & \ldots & a_{3 n} \\
\vdots & \vdots & \vdots & \ldots & \vdots & \vdots & \ldots & \vdots \\
a_{1(k-1)} & a_{2(k-1)} & a_{3(k-1)} & \ldots & a_{(k-1)(k-1)} & a_{(k-1)(k+1)} & \ldots & a_{n(k-1)} \\
a_{1(k+1)} & a_{2(k+1)} & a_{3(k+1)} & \ldots & a_{(k-1)(k+1)} & a_{(k+1)(k+1)} & \ldots & a_{n(k+1)} \\
\vdots & \vdots & \vdots & \ldots & \vdots & \vdots & \ldots & \vdots \\
a_{1 n} & a_{2 n} & a_{3 n} & \ldots & a_{n(k-1)} & a_{n(k+1)} & \ldots & a_{n n}
\end{array}\right] \\
& \text { e } \\
& b^{\prime}=\left[\begin{array}{llllllll}
a_{1 k} & a_{2 k} & a_{3 k} & \ldots & a_{(k-1)(k-1)} & a_{(k+1)(k+1)} & \ldots & a_{n k}
\end{array}\right]
\end{aligned}
$$

Queremos mostrar que

$$
b^{\prime} \cdot B^{-1}=\left[\begin{array}{llll}
-1 & -1 & \ldots & -1
\end{array}\right]
$$

Então, multiplicando à direita ambos os membros de (10) por $B$, temos: 


$$
b^{\prime}=\left[\begin{array}{llll}
-1 & -1 & \ldots & -1
\end{array}\right] B
$$

Tomando a transposta em ambos os lados de (11), obtemos:

$$
b=B^{\prime}\left[\begin{array}{llll}
-1 & -1 & \ldots & -1
\end{array}\right]^{\prime}=B\left[\begin{array}{llll}
-1 & -1 & \ldots & -1
\end{array}\right]^{\prime}
$$

Substituindo em (12) as expressões de $B$ e $b^{\prime}$, temos:

$\left[\begin{array}{rrrrrrr}a_{11} & a_{12} & \ldots & a_{1(k-1)} & a_{1(k+1)} & \ldots & a_{1 n} \\ a_{12} & a_{22} & \ldots & a_{2(k-1)} & a_{2(k+1)} & \ldots & a_{2 n} \\ a_{13} & a_{23} & \ldots & a_{3(k-1)} & a_{3(k+1)} & \ldots & a_{3 n} \\ \vdots & \vdots & \ldots & \vdots & \vdots & \ldots & \vdots \\ a_{1(k-1)} & a_{2(k-1)} & \ldots & a_{(k-1)(k-1)} & a_{(k-1)(k+1)} & \ldots & a_{n(k-1)} \\ a_{1(k+1)} & a_{2(k+1)} & \ldots & a_{(k-1)(k+1)} & a_{(k+1)(k+1)} & \ldots & a_{n(k+1)} \\ \vdots & \vdots & \ldots & \vdots & \vdots & \ldots & \vdots \\ -1 \\ a_{1 n} & a_{2 n} & \ldots & a_{n(k-1)} & a_{n(k+1)} & \ldots & a_{n n}\end{array}\right]\left[\begin{array}{r}-1 \\ \vdots \\ -1\end{array}\right]=\left[\begin{array}{r}a_{1 k} \\ a_{2 k} \\ \vdots \\ a_{(k-1)(k-1)} \\ a_{(k+1)(k+1)} \\ \vdots \\ a_{n k}\end{array}\right]$

$\mathrm{Ou}$

$$
\left[\begin{array}{r}
-\left(a_{11}+a_{12}+\ldots+a_{1(k-1)}+a_{1(k+1)}+\ldots+a_{1 n}\right) \\
-\left(a_{12}+a_{22}+\ldots+a_{2(k-1)}+a_{2(k+1)}+\ldots+a_{2 n}\right) \\
-\left(a_{13}+a_{23}+\ldots+a_{3(k-1)}+a_{3(k+1)}+\ldots+a_{3 n}\right) \\
\vdots \\
-\left(a_{1(k-1)}+a_{2(k-1)}+\ldots+a_{(k-1)(k-1)}+a_{(k-1)(k+1)}+\ldots+a_{n(k-1)}\right) \\
-\left(a_{1(k+1)}+a_{2(k+1)}+\ldots+a_{(k-1)(k+1)}+a_{(k+1)(k+1)}+\ldots+a_{n(k+1)}\right) \\
\vdots \\
-\left(a_{1 n}+a_{2 n}+\ldots+a_{n(k-1)}+a_{n(k+1)}+\ldots+a_{n n}\right)
\end{array}\right]=\left[\begin{array}{r}
a_{1 k} \\
a_{2 k} \\
\vdots \\
a_{(k-1)(k-1)} \\
a_{(k+1)(k+1)} \\
\vdots \\
a_{n k}
\end{array}\right]
$$

Mas, por hipótese,

$$
a_{i i}=-\sum_{i \neq j} a_{i j}, i, j=1,2, \ldots, n
$$



identidade. Assim,

$$
b \cdot B^{-1}=[-1,-1, \ldots,-1]
$$




\section{APÊNDICE C}

Consideremos uma seqüência $\left\{\beta_{k}\right\}$ de escalares positivos que converge para zero (WRIGHT, 1997), isto é,

$$
\lim _{k \rightarrow \infty} \beta_{k}=0 .
$$

Então,

1. $\left\{\beta_{k}\right\}$ converge Q-linearmente se existe um escalar $\beta \in(0,1)$ tal que

$$
\frac{\beta_{k+1}}{\beta_{k}} \leq \beta
$$

para todo $k$ suficientemente grande.

2. $\left\{\beta_{k}\right\}$ converge Q-superlinearmente se

$$
\lim _{k \rightarrow \infty} \frac{\beta_{k+1}}{\beta_{k}}=0 .
$$

3. $\left\{\beta_{k}\right\}$ converge Q-quadraticamente se existe uma constante $\mathrm{C}$ tal que

$$
\frac{\beta_{k+1}}{\beta_{k}^{2}} \leq C
$$

para todo $k$ suficientemente grande. 


\section{Bibliografia}

ALMEIDA, K. C. Estudos em Fluxo de Potência Ativa usando o Princípio do Mínimo Esforço. Campinas SP: [s.n.], 87. Dissertação de Mestrado, FEE - UNICAMP.

ATKINSON, K. An Introduction to Numerical Analysis. [S.l.]: John Wiley \& Sons, 1978.

AZEVEDO, A. T. Aplicação de Métodos de Pontos Interiores a Problemas de Manufatura e Engenharia Elétrica. Campinas - SP: [s.n.], 2002. Dissertação de Mestrado, FEEC UNICAMP.

BAZARAA, M. S.; JARVIS, J. J.; SHERALI, D. S. Linear Programming and Network Flows. New York: John Wiley \& Sons, 1990.

BEASLEY, J. E. Advances in Linear and Integer Programming. New York, US: OXFORD SCIENCE PUBLICATIONS, 1996.

CASTRONUOVO, E. D.; CAMPAGNOLO, J. M.; SAlGADO, R. New versions of interior point methods applied to the optimal power flow problem. Optimization Online Digest, p. 1-6, November, 2001.

ChIAVEGATO, F.; OliveirA, A. R. L.; SOARES, S. Pré-despacho de sistemas de energia elétrica via relaxação lagrangeana e métodos de pontos interiores. XXII CILAMCE, p. 1-14, 2001.

CLEMENTS, K. A.; DAVIS, P. W.; FREY, K. D. An interior point algorithm for weighted least absolute value power system state estimation. IEEE/PES Winter Meeting, v. 91-WM235-2PWRS, 1991.

CONTE, S. D.; BOOR, C. de. Elementary Numerical Analysis - An Algorithmic Approach. [S.l.]: McGraw-Hill International Book Company, 1981. 
DIKIN, I. I. Iterative solution of problems of linear and quadratic programming. Soviets Math. Doklady, v. 8, p. 674-675, 1967.

DUFF, I. S.; ERISMAN, A. M.; REID, J. K. Direct Methods for Sparse Matrices. Oxford: Clarendon Press, 1986.

EL-BAKRY, A. S. et al. On the formulation and the theory of the Newton interior-point method for nonlinear programming. Journal of Optimization Theory and Applications, v. 89 , p. $507-541,1996$.

FIACCO, A. V.; MCCORMICK, G. P. Nonlinear Programming: Sequential Unconstrained Minimization Techniques. [S.l.]: John Wiley \& Sons, 1968.

FREUND, R. M.; MIZUNO, S. Interior point methods: Current status and future directions. OPTIMA - Mathematical Programming Society Newsletter, n. 51, p. 1-28, 1996.

GOlUB, G. H.; LOAN, C. F. V. Matrix Computations Third Edition. Baltimore, Maryland: The Johns Hopkins University Press, 1996.

GULER, O.; YE, Y. Convergence behavior of interior-point algorithms. Mathematical Programming, v. 60, p. 215-228, 1993.

JENSEN, P. A.; BARD, J. F. Operations research models and methods. Page of Web. KARMARKAR, N. A new polynomial-time algorithm for linear programming. Combinatorica, v. 4, n. 4, p. 373-395, 1984.

KHACHIYAN, L. G. A polinomial algorithm in linear programming. Doklady Akademii Nauk SSSR, v. 224, p. 1093-1096, 1979.

KOJIMA, M. Basic lemmas in polynomial-time infeasible-interior-point methods for linear programs. Annals of Operations Research, v. 62, p. 1-28, 1996.

KOJIMA, M.; MEGIDDO, N.; MIZUNO, S. A primal-dual infeasible-interior-point algorithm for linear programming. Mathematical Programming, v. 61, p. 263-280, 1993. 
KOJIMA, M.; MIZUNO, S.; YOSHISE, A. A primal-dual interior point algorithm for linear programming. Mathematical Programming, p. 29-47, 1989.

LU, C. N.; UNUM, M. R. Network constrained security control using an interior point algorithm. IEEE Transactions on Power Systems, v. 8, p. 1068-1076, 1993.

MEGIDDO, N. Pathways to the optimal set in linear programming. Mathematical Programming, p. 131-158, New York, 1986.

MEHROTRA, S. On the implementation of a primal-dual interior point method. SIAM Journal on Optimization, v. 2, n. 4, p. 575-601, 1992.

MEHROTRA, S. On the implementation of a primal-dual interior point method. Siam J. Optmization, v. 2, n. 4, p. 575-601, November, 1992.

MIZUNO, S.; TODD, M. J.; YE, Y. On adaptive-step primal-dual interior-point algorithms for linear programming. Mathematics of Operations Research, v. 18, n. 4, p. 964-981, November, 1993.

MOMOH, J.; EL-HAWARY, M.; ADAPA, R. A review of selected optimal power flow literature to 1993, part I: Nonlinear and quadratic programming approaches. IEEE Transactions on Power System, v. 14, p. 96-104, 1999.

MONTEIRO, R. D. C.; ADLER, I. Interior path following primal-dual algorithms. part I: Linear programming. Mathematical Programming, n. 44, p. 27-41, 1989.

MONTICELLI, A.; GARCIA, A. Introdução a Sistemas de Energia Elétrica. Campinas, SP: Editora da UNICAMP, 1999.

MONTICELLI, A. et al. Interactive transmission network planning using a least effort criterion. IEEE Transactions on Power Apparatus and Systems, v. 10, p. 101, 1982.

OHISHI, T.; SOARES, S.; CARVALHO, M. F. Short term hydrothermal scheduling approach for dominantly hydro systems. IEEE Transactions on Power Systems, v. 6, p. 637-643, 1991. 
OLIVEIRA, A. R. L.; NEPOMUCENO, L.; SOARES, S. Optimal active power dispatch combining network flow and interior point approaches. IEEE Transactions on Power Systems, v. 18, p. 1235-1240, 2003.

OLIVEIRA, A. R. L.; NEPOMUCENO, L.; SOARES, S. Short term hydroelectric scheduling combining network flow and interior point approaches. Electrical Power $\mathscr{G}$ Energy Systems, v. 27, p. 91-99, 2005.

OLIVEIRA, A. R. L.; SOARES, S. Métodos de pontos interiores para problema de fluxo de potência ótimo. Anais do XIII Congresso Brasileiro de Automática, em CD-ROM, Florianópolis, SC, aceito para publicação na revista SBA: Controle $\&$ Automação, p. 790-795, 2000.

OLIVEIRA, A. R. L.; SORENSEN, D. C. A new class of preconditioners for large-scale linear systems from interior point methods for linear programming. Linear Algebra and Its Applications, v. 394, p. 1-24, 2005.

PANNAMBALAM, K.; QUINTANA, V. H.; VANNELLI, A. A fast algorithm for power system optimization problems using an interior point method. IEEE Transactions on Power Systems, v. 7, p. 892-899, 1992.

PENG, J.; ROOS, C.; TERLAKY, T. A new class of polynomial primal-dual methods for linear and semidefinite optimization. Optimization Online Digest-August, p. 1-1076, 2000.

PENG, J.; TERLAKY, T. A Dynamic Large-Update Primal-Dual Interior-Point Method for Linear Optimization. Ontario, Canada, 2001.

POTRA, F. A. A quadratically convergent predictor-corrector method for solving linear programs from infeasible starting points. Mathematical Programming, v. 67, p. 383-406, 1994.

POTRA, F. A.; WRIGHT, S. J. Interior point methods. Journal of Computational and Applied Mathematics, n. 124, p. 281-302, 2000. 
QUINTANA, V. H.; TORRES, G. L.; PALOMO, J. M. Interior-point methods and their applications to power systems: A classification of publications and software codes. IEEE Transactions on Power Systems, v. 15, p. 170-176, 2000.

ROOS, C. A full-newton step o(n) infeasible interior-point algorithm for linear optimization. Optimization Online Digest, p. 1-26, June, 2005.

SALAHI, M.; PENG, J.; TERLAKY, T. On mehrotra-type predictor-corrector algorithms. Optimization Online Digest, p. 1-18, April, 2005.

SILVA, R.; SOARES, J.; VICENTE, L. N. Local analysis of the feasible primal-dual interior-point method. Optimization Online Digest, p. 1-13, March, 2005.

SOARES, S.; SALMAZO, C. T. Minimum loss predispatch model for hydroelectric systems. IEEE Transactions on Power Systems, v. 12, n. 3, p. 1220-1228, 1997.

SPORRE, G. On Some Properties of Interior Methods for Optimization. Stockholm: [s.n.], 2003. Doctoral Thesis.

STOJOKOVIC, N. V.; STANIMIROVIC, P. S. Initial point in primal-dual interior point method. Mechanics, Automatic Control and Robotics, v. 3, n. 11, p. 219-222, 2001.

TERLAKY, T. Interior Point Methods of Mathematical Programming. London: Kluwer Academic Publishers, 1996.

VANDERBEI, R. J. Linear Programming - Foundations and Extensions. Boston, USA: Kluwer Academics Publishers, 1996.

VARGAS, L.; QUINTANA, V.; VANNELLI, A. A tutorial description of an interior point method and its application to security constrained economic dispatch. IEEE Transactions on Power Systems, v. 11, p. 1315-1324, 1993.

WRIGHT, S. J. Primal-Dual Interior-Point Methods. Philadelphia: SIAM, 1997.

WRIGHT, S. J. Recent developments in interior-point methods. Proceedings of 19th IFIP TC7 Conference on System Modelling and Optmization, p. 1-23, July, 1999. 